University of Rhode Island

DigitalCommons@URI

Open Access Master's Theses

2017

\title{
Investigating Lateral Line Canal Morphogenesis as a Bone Remodeling Process in Lake Malawi Cichlids
}

Julia W. Johnstone

University of Rhode Island, jwjohnstone@my.uri.edu

Follow this and additional works at: https://digitalcommons.uri.edu/theses

\section{Recommended Citation}

Johnstone, Julia W., "Investigating Lateral Line Canal Morphogenesis as a Bone Remodeling Process in Lake Malawi Cichlids" (2017). Open Access Master's Theses. Paper 1074.

https://digitalcommons.uri.edu/theses/1074

This Thesis is brought to you for free and open access by DigitalCommons@URI. It has been accepted for inclusion in Open Access Master's Theses by an authorized administrator of DigitalCommons@URI. For more information, please contact digitalcommons-group@uri.edu. 
INVESTIGATING LATERAL LINE CANAL MORPHOGENESIS AS A BONE REMODELING PROCESS IN LAKE MALAWI CICHLIDS

BY

JULIA W. JOHNSTONE

A THESIS SUBMITTED IN PARTIAL FULFILLMENT OF THE REQUIREMENTS FOR THE DEGREE OF

MASTER OF SCIENCE

IN

BIOLOGICAL AND ENVIRONMENTAL SCIENCES

UNIVERSITY OF RHODE ISLAND 
MASTER OF SCIENCE THESIS

OF

JULIA W JOHNSTONE

APPROVED:

Thesis Committee:

Major Professor: Jacqueline F. Webb

Steven Q. Irvine

Bethany Jenkins

Nasser H. Zawia

DEAN OF THE GRADUATE SCHOOL

UNIVERSITY OF RHODE ISLAND

2017 


\begin{abstract}
The lateral line (LL) system is a key non-visual sensory modality founded in all fishes and larval and adult aquatic amphibians. In bony fishes, the LL system consists of series of superficial neuromasts (on the skin surface) and canal neuromasts (housed in bony canals). Differences in LL canal phenotype have consequences for LL sensory capabilities, and, as such, could contribute to speciation. The LL system develops with a process of neuromast patterning followed by the genesis of canals from dermal bone around a subset of neuromasts. This study used histochemical methods to describe canal morphogenesis by assessing osteoblast (OB, bone building cells) and osteoclast (OC, bone resorbing cells) activity through ontogeny. OB and OC activity was studied along the length and around the circumference of the mandibular LL canal in two species of Lake Malawi cichlids. Aulonocara stuartgranti (widened canals) and Tramitichromis sp. (narrow canals) are known to develop their different canal phenotypes as a result of dissociated heterochrony. In both species, OB activity is concentrated in the canal roof and floor (behind the neuromast), while OC activity is focused primarily in the canal walls. Further, both species demonstrate one pulse of bone deposition early in ontogeny and another pulse of bone resorption later in ontogeny, but in Aulonocara not Tramitichromis, the pulse of bone resorption is accompanied by high levels of $\mathrm{OB}$ activity. Thus, the second pulse of bone cell activity in Aulonocara is defined by bone resorption and bone deposition: active bone remodeling by the simultaneous action of OBs and OCs. These results corroborate the observation of dissociated heterochrony (Bird and Webb, 2014) at the cell level, suggesting that the nature of neuromast-centered canal morphogenesis may differ depending on LL canal phenotypes.
\end{abstract}




\section{ACKNOWLEDGEMENTS}

I would like to thank my friends and family for their constant, continuing support - in particular, my lab mates, Ashley Marranzino and Andrea Schlunk for their companionship, Yinan $\mathrm{Hu}$ for his help with experimental troubleshooting and his comments as my thesis came together, and the Webb lab undergraduates who helped me maintain the fish stocks from which I took my samples, Katie Nickles, Sean Duffey, Matt Gibson, Danielle Jordan, and Emma Shoemaker. Above all I would like to thank my advisor, Dr. Jacqueline Webb, for her insight, guidance, and patience - I am truly grateful and have learned so much. I would also like to acknowledge the sources of my financial support: the College of the Environment and Life Sciences, URI; the Vice President of Academic Affairs and Economic Development; and the URI Graduate School. 


\section{PREFACE}

This thesis is composed in Manuscript format for submission to Developmental Dynamics. 


\section{TABLE OF CONTENTS}

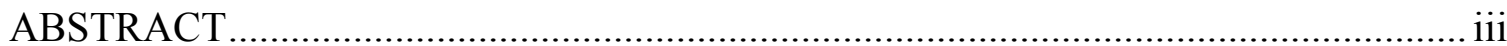

ACKNOWLEDGEMENTS ............................................................................... ii

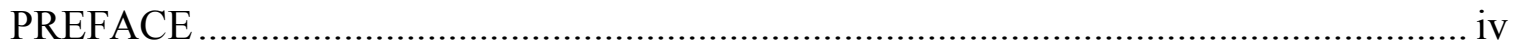

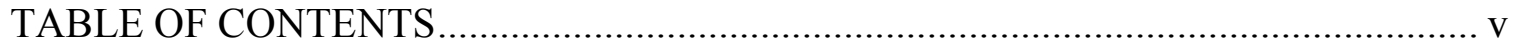

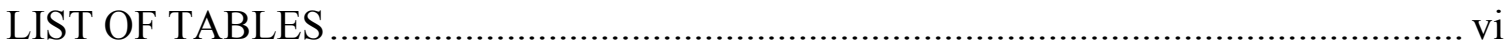

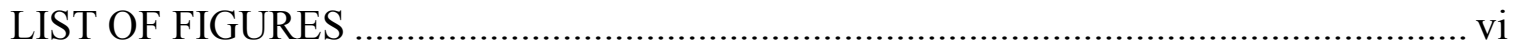

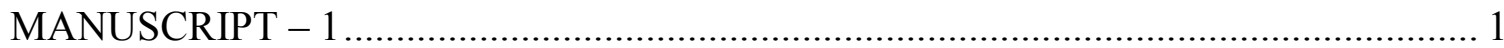

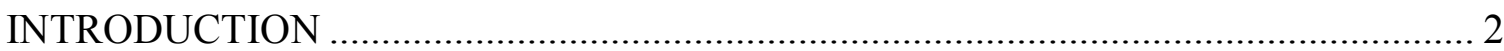

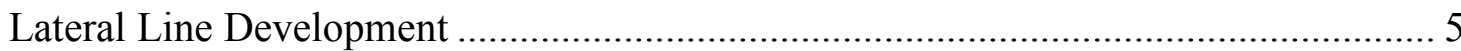

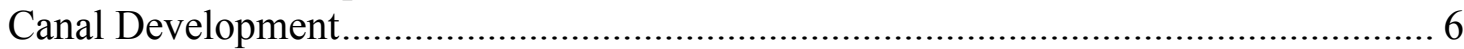

Dermal Bone Development and Remodeling ......................................................... 7

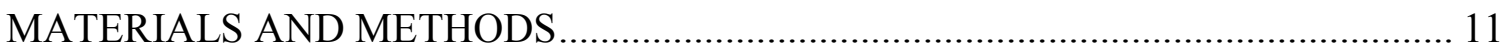

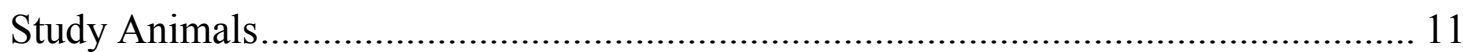

Fluorescent Vital Staining for Bone and Neuromasts......................................... 12

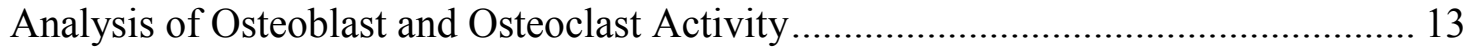

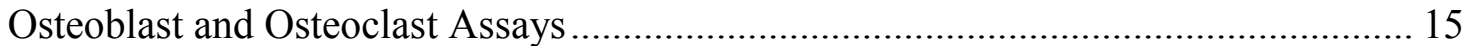

Quantification and Visualization of Osteoblast and Osteoclast Activity Data ............ 18

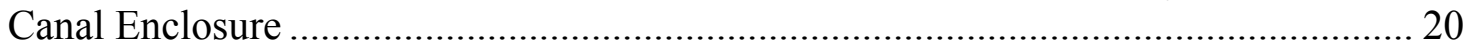

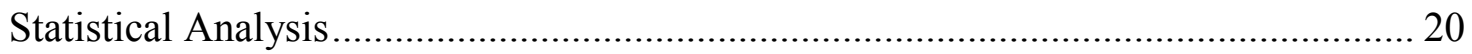

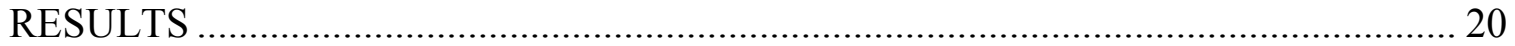

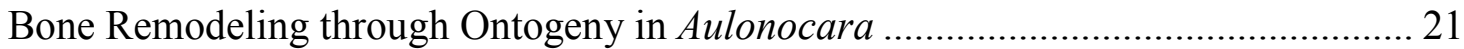

Bone Remodeling through Ontogeny in Tramitichromis ........................................ 24

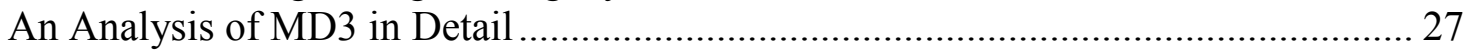

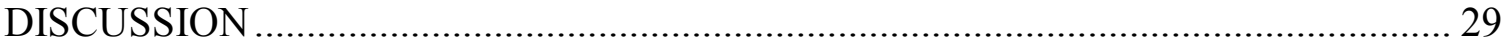

Osteoblasts and Osteoclast Activity in Canal Growth ............................................ 31

Evidence of Neuromast-Centered Canal Morphogenesis at the Cell Level ................. 33

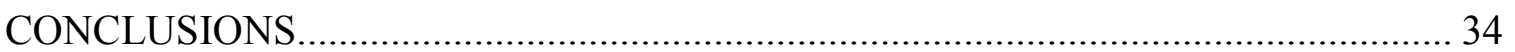

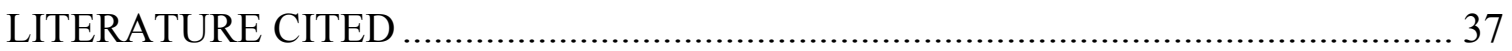




\section{LIST OF TABLES}

TABLE

PAGE

Table 1. Results of Chi Square Tests of Uniform Radial Distribution of Osteoclast

Nuclei in Aulonocara stuartgranti.

Table 2. Results of Chi Square Tests of Uniform Radial Distribution of Osteoclasts in Tramitichromis sp .42

\section{LIST OF FIGURES}

FIGURE

PAGE

Figure 1. Distribution of neuromasts on the head of Aulonocara stuartgranti and Tramitichromis sp.

Figure 2. Five patterns of cranial canal systems found among teleost fishes.

Figure 3. Widened and narrow canals in the mandible of Tramitichromis sp. and Aulonocara stuartgranti.

Figure 4. Anatomy of a neuromast. .46

Figure 5. Neuromast-centered canal morphogenesis and fusion of adjacent canal segments.

Figure 6. Stages of neuromast-centered canal morphogenesis.

Figure 7. Distribution of canal neuromasts in the mandibular canal in relation to the positions defined for analysis

Figure 8.. Method for analysis of osteoblast and osteoclast activity using AP and TRAP staining. .50

Figure 9. Osteoblast and osteoclast activity in the mandibular canal of Aulonocara 
stuartgranti

Figure 10. Vital stains used to simultaneously visualize bone and neuromasts ......52

Figure 11. Vital and histochemical staining methods in $16 \mathrm{~mm} \mathrm{SL}$

Tramitichromis sp. .53

Figure 12. Whole mount staining for osteoblast and osteoclast activity. 54

Figure 13. Ring of AP staining around superficial and canal neuromasts. .55

Figure 14. Osteoblast and osteoclast activity through ontogeny in Aulonocara stuartgranti .56

Figure 15. Osteoblast activity through ontogeny along canal length (MD2-MD4) in Aulonocara stuartgranti.

Figure 16. Osteoclast activity through ontogeny along canal length in Aulonocara stuartgranti 58

Figure 17. Total osteoblast and osteoclast activity through ontogeny in Tramitichromis sp. .59

Figure 18. Osteoblast activity through ontogeny along canal length in Tramitichromis sp. .60

Figure 19. Osteoclast activity through ontogeny along canal length in

Tramitichromis sp.

Figure 20. Osteoblast and osteoclast activity in the MD3 region in $16 \mathrm{~mm} \mathrm{SL}$ Aulonocara stuartgranti .62

Figure 21. Osteoblast activity in MD3 (Positions 17-21) through ontogeny in Aulonocara stuartgranti.

Figure 22. Osteoclast activity in MD3 (Positions 17-21) through ontogeny in 
Figure 23. Osteoblast activity in MD3 (Positions 17-21) through ontogeny in Tramitichromis sp .65

Figure 24. Osteoclast activity in MD3 (Positions 17-21) through ontogeny in Tramitichromis sp. .66

Figure 25. Canal development in neuromast regions MD2, MD3, and MD4 in Aulonocara stuartgranti.

Figure 26. Canal development in neuromast regions MD2, MD3, and MD4 in Tramitichromis sp. .68

Figure 27. Osteoblast and osteoclast activity through ontogeny in Aulonocara stuartgranti and Tramitichromis sp.

Figure 28. Neuromast-centered canal morphogenesis in $12 \mathrm{~mm}$ SL Aulonocara stuartgranti and Tramitichromis sp.

Figure 29. Osteoblast activity in $12 \mathrm{~mm}$ SL Aulonocara stuartgranti and Tramitichromis sp. along canal length in each quadrant .......70 


\section{MANUSCRIPT - 1}

Prepared for submission to Developmental Dynamics.

Investigation of Lateral Line Canal Morphogenesis as a Bone Remodeling Process in Two Lake Malawi Cichlids

Julia W Johnstone, Jacqueline F Webb

Biological Sciences, University of Rhode Island, Kingston, RI, USA

Corresponding Author: Jacqueline F Webb

Biological Sciences

University of Rhode Island

280, Center for Biotechnology and Life Sciences

120 Flagg Rd

Kingston, RI, 02881, USA

Phone: +1-401-874-2609

Email address: jacqueline_webb@uri.edu 


\section{INTRODUCTION}

The mechanosensory lateral line system senses water flow, enabling fishes to respond to hydrodynamic cues involved in prey detection, predator avoidance, communication, and navigation (Dijkgraaf 1963; Janssen 1997; Webb et al. 2008; Schwalbe et al. 2012; Yanase et al. 2014; Schwalbe and Webb 2015). The sensory organs of the lateral line system, the neuromasts, are found in stereotyped locations on the surface of the skin (superficial neuromasts) and in hollow canals integrated in a conserved subset of dermal bones on the head and in a linear series of tubed scales on the trunk (canal neuromasts; Webb 2014a, b). Superficial and canal neuromasts function as velocimeters and accelerometers, respectively (McHenry and Liao 2014). Neuromasts are arranged in conserved patterns on the head and trunk in larvae (Gompel et al. 2001; Webb and Shirey 2003; Ghysen and Dambly-Chaudiere 2004; Thomas et al. 2015; Becker et al. 2016; Figure 1), but cranial canal phenotypes vary among juvenile and adult fishes of different species (reviewed in Webb 2014b).

Five cranial canal morphologies are found among bony fishes: narrow-simple (referred to as "narrow" from here on), narrow-branched, narrow with widened tubules, widened, and reduced (reviewed in Webb 2014b; Figure 2). The functional consequences of some aspects of this morphological variation have been investigated (Denton and Gray 1988; Janssen 1997; Van Snick Gray and Stauffer 2004; Schwalbe and Webb 2013; Webb 2014a, b; Schwalbe and Webb 2015; Klein and Bleckmann 2015; Schwalbe et al. 2016). For example, widened canals are more sensitive to low frequencies $(<60 \mathrm{~Hz})$ than narrow canals, and this increased sensitivity plays a role in prey detection (in the water 
column and in the benthos). However, the increased resonance in the widened canals results in a slower reaction time than in narrow canals (reviewed in Coombs et al. 1992). Thus, differences in canal morphology confer functional differences in the lateral line system and are therefore ecologically and evolutionarily relevant.

The diversity of fishes in the Family Cichlidae presents a unique opportunity to compare different lateral line canal phenotypes in closely related taxa. The rapid evolutionary diversification of cichlids is a well-known example of explosive speciation; over 1000 species of cichlids have arisen during the last million years in Lake Malawi and Lake Victoria together (Allender et al. 2003). Many of these species occupy partially overlapping physical ranges and ecological niches, but they still sort into discrete species in nature, despite their ability to produce viable hybrid offspring in lab settings (Allender et al. 2003; Seehausen et al. 2008). Attempts to understand the biological mechanisms underlying cichlid adaptive speciation have been a major focus of evolutionary biologists and geneticists (e.g., Allender et al. 2003; Kocher 2004; Van Snick Gray and Stauffer 2004; Albertson et al. 2005; Seehausen et al. 2008; Cooper et al. 2011; Parsons et al. 2012; Powder et al. 2015). Variation in sensory ability among species (e.g. vision) has been shown to underlie differences in behavior, which provides a basis for speciation along environmental gradients (Allender et al. 2003; Schleuter and Eckmann 2006; Seehausen et al. 2008). In Lake Malawi, one such environmental gradient is the decrease in light intensity with depth. Deeper-living cichlid species need to rely more on nonvisual sensory modalities such as the mechanosensory lateral line system to find prey in deeper, darker waters. Related shallow-water cichlids live in a brighter environment, so the selective pressures associated with light limitation is relaxed, leaving the shallow- 
water species free to rely less on their lateral line and more on visual prey detection. Taking this example, phenotypic differences in lateral line canal morphology with functional consequences for sensory capability could have contributed to the rapid adaptive speciation of this group. However, any consideration of that possibility would need to be grounded in a robust understanding of the link between lateral line structure and function, particularly the differences between canal phenotypes.

The two Lake Malawi cichlids Aulonocara stuartgranti and Tramitichromis sp. (referred to by genus name throughout) are an ideal pair of species that can be used to investigate the developmental and functional differences between lateral line phenotypes (e.g. Schwalbe et al., 2012; Schwalbe and Webb 2013; Bird and Webb, 2014; Schwalbe and Webb 2015; Schwalbe, et al. 2016; Becker et al. 2016). Both species locate prey in sandy-bottomed environments, but Aulonocara has widened canals and members of the genus live at a greater range of depths $(5-120 \mathrm{~m})$, while Tramitichromis has narrow canals and tends to live in relatively shallow waters ( $>15$ m; Fryer and Iles 1972; Konings 1990, 2007; Figure 3). Recent studies have demonstrated that while both Aulonocara and Tramitichromis employ their lateral line systems in combination with vision to detect prey in light environments, only Aulonocara uses its lateral line to detect prey and feed in dark environments below a critical light level (Schwalbe et al. 2012; Schwalbe and Webb 2013; Schwalbe and Webb 2015; Schwalbe et al. 2016). Further, the generation of interspecific differences in canal phenotype are a result of dissociated heterochrony during canal morphogenesis (Bird and Webb 2014). 


\section{Lateral Line Development}

In bony fishes, lateral line development occurs in three phases, beginning in the embryo and continuing until long after metamorphosis from the larval stage to the juvenile stage. In the embryo, neuromast primordia migrate (on the trunk) or elongate (on the head) from cranial ectodermal lateral line placodes, establishing the distribution of neuromasts (Northcutt and Gans 1983, reviewed in Webb 2014b). The neuromasts then grow and mature, and some superficial neuromasts start to proliferate. Finally, a subset of neuromasts is enclosed in bony canals through the process of neuromast-centered canal morphogenesis (reviewed in Webb 2014b).

Each neuromast is composed of a central group of hair cells surrounded by nonsensory support and mantle cells (Figure 4a). A ciliary bundle extends from the apical surface of each hair cell, consisting of one long kinocilium adjacent to several, graded stereocilia. The location of the kinocilium in relation to the stereocilia defines the polarity, or axis of best physiological sensitivity, of each hair cell, and the presence of hair cells with like and opposing polarities ( $180^{\circ}$ to each other) defines the axis of best physiological sensitivity of a neuromast (discussed in Webb 2014b). In juvenile and adult fishes, the neuromast surface occupied by hair cells is restricted to a small round or oval area known as the sensory strip (Coombs et al. 1988; Figure 4b). Basally, the hair cells are innervated by sensory neurons, and apically, the ciliary bundles project into a protective gelatinous cupula, which is thought to be secreted by the support or mantle cells (Münz 1979; Blaxter 1987; Webb 2014b). The cupula is flexible enough to be displaced by hydrodynamic stimuli, thus enabling a neuromast to respond to water flows and vibrations (Van Trump and McHenry 2008; Windsor and McHenry 2009). Hair cells 
are regenerated throughout the life of the fish, making the lateral line system a promising model for research into questions of sensory organ regeneration, especially as it relates to human deafness (Dambly-Chaudiere et al. 2003; Chitnis et al. 2011; Cruz et al. 2015). The posterior (trunk) lateral line of zebrafish (Danio rerio), in particular, has been the focus of many studies aiming to tease apart the genetic control of lateral line patterning and hair cell regeneration (Raible and Kruse 2000; Gompel et al. 2001; Sapède et al. 2002; López-Schier et al. 2004; Ma and Raible 2009; Romero-Carvajal et al. 2015).

\section{Canal Development}

The pattern and timing of canal morphogenesis have been described in a number of teleost taxa (reviewed in Webb 2014b). Canal development occurs in four stages, a model that has been used in several studies (Tarby and Webb 2003; Webb and Shirey 2003; Moore 2008; Bird and Webb 2014; Carter 2014; Figure 5). It has been noted by several authors that canals start to form in the vicinity of neuromasts, a phenomenon termed "neuromast-centered canal morphogenesis" (reviewed in Webb 2014b). Canal morphogenesis begins with a presumptive canal neuromast sitting on the skin surface (Stage I). The neuromast appears to sink into a depression (Stage IIa) and canal bone starts to ossify within the dermis, forming ridges, which grow upward to form canal walls around the neuromast (Stage IIb). Soft tissue (epidermis, dermis) fuses over the neuromast (Stage III). The bony walls continue to grow within the dermis and over the neuromast, finally fusing to form the ossified roof of the canal segment (Stage IV). From above, the canal walls appear to form "scallops" of soft tissue, with bone within (described by Webb 2014a, 2014b, Bird and Webb 2014). In histological cross sections, 
two growing arms of soft tissue containing bone reach around the canal circumference as growing bony fronts, which then fuse over the neuromast (Figures 5, 6). As individual canal segments form, the walls of adjacent canal segments fuse, leaving a pore in the canal roof between them. This process forms a continuous canal in which each neuromast sits in an ossified canal segment, between which there are pores that connect the canal with the outside environment. As the fish grows, canal diameter increases and the canals remain integrated within the dermal bones (Bird and Webb 2014), but this process has not been investigated at the cell level.

\section{Dermal Bone Development and Remodeling}

All of the bones associated with the cranial lateral line canals in teleosts are dermal bones, meaning they are formed directly within the dermis without a cartilage model (Cubbage and Mabee 1996; Weigele and Franz-Odendaal 2016). In addition (with a few notable exceptions) all "higher" teleosts, including cichlids, have acellular bone (Huysseune 2000). Thus, the bones that house the lateral line canals in cichlid fishes are dermal, acellular bones (Huysseune 2000). Acellular dermal bone has several characteristic features including mode of ossification, absence of osteocytes embedded in the bone matrix, and the manner in which the bone is resorbed or remodeled. The osteogenesis of endochondral bone involves a preliminary cartilage template composed of chondrocytes, which is secreted by mesenchymal cells. In contrast, dermal bones do not form from a cartilaginous template, but form directly within the dermis. Secondly, in cellular endochondral bone, osteoblasts (bone building cells) begin mineralizing the template from the inside and become trapped in the bone matrix (Weigele and Franz- 
Odendaal 2016). Once they are surrounded by bone matrix, the osteoblasts are termed osteocytes, and their presence in the bone identifies the bone as cellular (Moss 1960; Weiss and Watabe 1979; Weigele and Franz-Odendaal 2016). In acellular bones, including acellular dermal bones, the osteoblasts form mineralized bone in a polarized manner, retreating from the ossification front so that they do not become entrapped in bone matrix (Ekanayake and Hall 1988).

A third feature of acellular dermal bone is the manner in which it is usually resorbed or remodeled. There are two types of osteoclasts (bone resorbing cells) mononucleated osteoclasts, which are associated with shallow sites of resorption (without obvious lacunae), and multinucleated osteoclasts, which are associated with deep lacunar resorption sites (Witten 1997; Witten and Huysseune 2009). Unlike resorption of cellular bone (e.g., in mammals), which is characterized by multinucleated osteoclasts and deep lacunae, resorption of dermal bone in teleost acellular bone is likely to be the site of shallow bone resorption carried out by mononucleated osteoclasts (Witten 1997). However, in times of intense bone remodeling, these mononucleated osteoclasts may group together to resemble large multinucleated osteoclasts. Under other conditions, however, they may appear flat, similar to the bone-lining cells in mammals (Witten 1997).

In prior investigations, the presence of any type of osteoclast has been considered to be a hallmark of such remodeling in acellular bone (Witten and Villwock 1997; Nemoto et al. 2007). Osteoclast activity has been found primarily in bony elements undergoing growth, such as those in the craniofacial skeleton and vertebral column (Witten et al. 2001). The growth of the dentary bone (the dermal bone which houses the 
mandibular lateral line canal, and which composes the majority of the lower jaw of fishes) was examined for bone resorption and remodeling by Witten, Hansen and Hall (2001). It was found to be the site of resorption by both mono- and multinucleated osteoclasts, with an ontogenetic progression from mononucleated osteoclasts in 20-day old zebrafish to multinucleated osteoclasts in 40-day old fish (Witten et al. 2001). The mandibular lateral line canal, though not expressly assayed for bone remodeling in that study, was noted as an area for further study and probable bone remodeling.

The processes of acellular dermal bone ossification and remodeling (particularly in growing skeletal elements), the ontogenetic increases in lateral line canal diameter, and the observations of osteoclasts in the vicinity of lateral line canals suggest that the cranial lateral line canals are active sites of bone remodeling. The presence of osteoclasts was noted in cranial lateral line canals in a cichlid fish (Witten 1997), and their activity has been suggested by an observed increase in canal diameter that began even before the completion of canal morphogenesis (Tarby and Webb, 2003; Bird and Webb, 2014). The presence of osteoclasts in the cranial lateral line canals of zebrafish during canal morphogenesis was confirmed (Moore 2006), showing that the majority of osteoclasts were localized to the inside surface of the canals. Bone remodeling was demonstrated in the posterior (trunk) lateral line canal of the zebrafish by the presence of osteoclasts (Wada, et al. 2014). However, no study has yet investigated the activity of osteoblasts in the bone remodeling process in teleost fishes despite their role as "builders" of the canals. Thus, it is hypothesized that the combination of the activity of osteoblasts and of osteoclasts is responsible for the initial morphogenesis and subsequent growth (increase in canal diameter) of the lateral line canals, and further, that differences in the dynamics 
of these two cell types during larval and post-larval (juvenile) development can explain variation in adult canal phenotype among species. Also, based on the concept of neuromast-centered canal morphogenesis, it is predicted that osteoblast and osteoclast activity will differ along the canal length in relation to the location of neuromasts. In addition, it has been established that heterochronic shifts in the process of canal enclosure explain the morphological differences between narrow and widened canals (Bird and Webb, 2014). A delay in the commencement of canal morphogenesis coupled with an acceleration in the increases in canal diameter and neuromast size resulted in the development of a widened rather than a narrow canal phenotype in cichlids (Bird and Webb 2014). However, the dynamics of osteoblast and osteoclast activity responsible for the construction of divergent adult canal phenotypes (e.g., narrow vs. widened) has not been examined.

Thus, the goal of this work was to determine: (1) how the activity of osteoblasts and osteoclasts could account for the initial enclosure of the mandibular lateral line canals (including exploration of neuromast-centered canal morphogenesis) and the increase in mandibular canal diameter with fish growth, and (2) how the activity of osteoblasts and osteoclasts contributes to divergent adult lateral line canal phenotypes (widened, narrow) in two species of cichlids: Aulonocara stuartgranti and Tramitichromis sp. A comparison of spatial patterns of bone deposition and resorption during canal morphogenesis in species with different adult canal morphologies provides a key link between activity at the cell-level, adult phenotype, and sensory function. Understanding the dynamics of canal formation and growth will lay the foundation for future studies of the genetic mechanisms underlying canal morphogenesis. More 
generally, the analysis of osteoblast and osteoclast activity and distribution during development will contribute to our understanding of the contribution of acellular bone skeletogenesis and its contribution to post-embryonic craniofacial development in fishes.

\section{MATERIALS AND METHODS}

\section{Study Animals}

Breeding groups of the maternal mouth-brooding cichlids, Aulonocara stuartgranti and Tramitichromis sp. reproduce regularly in the lab and have been used in prior studies of lateral line morphology and development, and lateral line-mediated behavior (Schwalbe et al. 2012; Bird and Webb 2014; Schwalbe and Webb 2014; Becker et al. 2016; Schwalbe et al. 2016). To obtain larvae for analysis, broods of recently hatched fry were removed from mothers' mouths at $\sim 9$-days post fertilization (dpf) and raised in round-bottom flasks with flowing water within small tanks in an AHAB flow through system (Aquatic Habitats Inc.). As fry absorbed their yolk sacs they were able to swim out of the flasks into the surrounding tanks. Fry were fed twice a day, first with plankton pellets (Hikari ${ }^{\circledR}$ Middle Larval Stage Plankton), then flake food (an equal mixture of earthworm, egg yolk, and Spirulina flakes, Pentair Aquatic Eco-Systems, Inc), and then cichlid pellets (NewLife Spectrum Sinking Formula Cichlid Pellets). Aulonocara were sampled from 11 broods (AuHb-B156, -B160, -B163, -B164, -B167, B173, -B177, -B180, -B181, -B183, -B184) and Tramitichromis were sampled from 8 broods (TRA-B062, -B065, -B066, -B067, -B068, -B071, -B072, -B073), which were reared from February 2015 to March 2016. Fish were anaesthetized with $0.02 \%$ solution of MS-222 (ethyl 3-aminobenzoate methanesulfonate; Sigma-Aldrich-Aldrich) in tank 
water, then fixed in 4\% paraformaldehyde (Electron Microscopy Sciences) in phosphate buffered saline (PBS). These fish were used to generate ontogenetic series for fluorescent vital staining and histochemical assays of osteoblast and osteoclast activity. All work was done under an approved URI IACUC Protocol (\#AN-08-11-005).

\section{Fluorescent Vital Staining for Bone and Neuromasts}

An ontogenetic series of each study species (Aulonocara stuartgranti and Tramitichromis sp.) was vitally stained and analyzed to visualize canal ossification and canal neuromasts in order to identify the size classes of larvae and juveniles that would subsequently be used for histochemical assays of osteoblast and osteoclast activity.

Live fish were immersed in 0.0024\% 4-di-2-ASP (Sigma-Aldrich D0815) for 5 minutes in the dark, then moved to $0.05 \%$ calcein (Sigma-Aldrich C0875) for 5 minutes in the dark to simultaneously visualize neuromasts and newly calcified bone, respectively. Fish were rinsed in tank water, and anaesthetized in $0.02 \%$ MS-222 in tank water (based on Fujimura and Okada, 2008) and immediately imaged with epifluorescence on a dissecting scope (Nikon SMZ 1500) with a camera (SPOT RT3 25.2 2MP color mosaic) using SPOT 5.2 imaging software. The DS-Red filter (excitation $\lambda=545 \mathrm{~nm}$ ) was used to visualize hair cells in neuromasts, and the GFP filter (excitation $\lambda=470 \mathrm{~nm}$ ) was then used to image calcein staining of newly calcified bone. Images taken at multiple focal planes were merged using Helicon Focus (Helicon Soft LTD). Images of the same individual revealing neuromasts (orange) and newly calcified bone (green), respectively, were combined in Photoshop (Adobe Systems Incorporated) to reveal the location of the canal neuromasts within the ossifying mandibular canal. After imaging, 
fish were fixed in $4 \%$ paraformaldehyde in PBS and stained with methylene blue $(0.05 \%$ aqueous solution) to highlight the stage of formation of individual canal segments. This procedure allowed the developmental stage of each canal segment to be assessed (according to Webb and Shirey, 2003). After assessing the degree of canal development in all five size classes (8-27 mm SL), it was determined that canals had not yet begun to form (Stage I) at $8 \mathrm{~mm} \mathrm{SL}$, and that all canal segments were already enclosed and ossified (Stage IV) at $24 \mathrm{~mm} \mathrm{SL}$. After determining these end points, three additional intermediate size classes were identified: 12,16 , and $20 \mathrm{~mm}$ SL. Thus, it was determined that five size classes $(8,12,16,20$, and $24 \mathrm{~mm} \mathrm{SL},+/-1 \mathrm{~mm})$ would be used for histochemical analysis of canal morphogenesis and growth through a process of bone remodeling.

\section{Analysis of Osteoblast and Osteoclast Activity}

Osteoblast and osteoclast activity was analyzed in the mandibular (MD) canal on the right side of the head only. The MD canal runs rostro-caudally, thereby allowing for accurate measurements of canal and neuromast dimensions in serial transverse sections (as in prior studies: Bird and Webb 2014; Webb et al. 2014; Becker et al. 2016).

The MD canal in both Aulonocara and Tramitichromis contains five neuromasts (MD1-5). MD1 was excluded because it is the last to enclose and because in very young larvae it can be difficult to distinguish from the nearby superficial neuromasts, which are similar in size. MD5 is located in the anguloarticular bone whereas MD1-4 are in the dentary bone, so MD5 was eliminated from the analysis to avoid any confounding factors due to its association with a different bone. Thus, the portion of the canal in the dentary 
bone containing neuromasts MD2, MD3, and MD4 was used for analysis. Osteoblast and osteoclast activity was analyzed at the location of each neuromast as well as in the regions between neuromasts (e.g., "mid 2-3", "mid 3-4”). In addition, a more detailed assessment of osteoblast and osteoclast dynamics in association with a single neuromast (MD3) was carried out in an ontogenetic series of each of the two study species.

An initial inspection of histological material revealed the positions of mandibular canal neuromasts (MD1-5). Neuromast length (in the rostro-caudal axis) was determined by counting the number of sections in which neuromast was present $\mathrm{x}$ thickness of the sections. A total of 29 positions along the rostro-caudal length of the canal, based on their proximity to the canal neuromasts, were defined for analysis. Positions were located at the rostral end of each neuromast, and then at locations $25 \%, 50 \%, 75 \%$ along the length of the neuromast and at the caudal end of the neuromast, as well as the midpoint, and flanking quarter points between two adjacent neuromasts (Figure 7). Positions 9-13, 1721, and 25-29 are referred to as the MD2, MD3, and MD4 Regions, respectively, and Positions 14-16 and 22-24 are referred to as the mid 2-3 and mid 3-4 Regions, respectively. As such, the area of interest for this study is defined by five Regions along the MD canal - the MD2 Region, the mid 2-3 Region, the MD3 Region, the mid 3-4 Region, and the MD4 Region (see Figure 7). The MD2, MD3, and MD4 Regions are referred to collectively as the "neuromast-associated" Regions, and the mid 2-3 and mid 3-4 Regions as the "inter-neuromast” Regions.

Further, histological sections corresponding to each of the 29 positions along the length of the canal were used for the analysis of the spatial distribution of osteoblast and osteoclast activity around the canal circumference. To accomplish this, the canal 
circumference was divided into 8 radial segments (Figure 8) and a transparency with a radial grid divided into 8 equal segments was placed over a live image of a histological section on a computer screen with the central axis aligned with the center of the neuromast. In sections where the canal was not completely ossified, the location of the advancing bone fronts (growing canal walls) was also recorded, as was the developmental stage of the canal segment (Stage 1, 2a, 2b, 3, 4; as per Tarby and Webb, 2003). After preliminary analysis, it was determined that data from pairs of the eight segments could be combined to form four quadrants, with Quadrant 1 representing the canal floor/neuromast, Quadrants 2 and 3 representing the left and right canal walls, respectively, and Quadrant 4 representing the canal roof (Figure 8c,d).

\section{Osteoblast and Osteoclast Assays}

To visualize osteoblast and osteoclast activity during canal morphogenesis, fish were stained either for alkaline phosphatase (AP), an enzyme expressed by active osteoblasts and thereby found in areas of bone ossifiation, or for tartrate-resistant acid phosphatase (TRAP), an enzyme expressed by active osteoclasts and thus found in areas undergoing bone resorption.

In each species, three fish in each of five size classes $(8,12,16,20,24 \mathrm{~mm} \mathrm{SL} \pm 1$ $\mathrm{mm}$ ) were stained for AP and three fish in each size class were stained for TRAP. Fish size was used to define size classes because canal development tracks with size, rather than age (Münz 1979, Ledent 2002). Fish were anaesthetized in 0.02\% MS-222, then fixed in 4\% paraformaldehyde in PBS for 12 - 24 hours. If the staining assay (either AP or TRAP) could not be performed immediately after fixation, samples were dehydrated in 
a graded ethanol series to $70 \%$ ethanol for storage until they could be stained (Edsall and Franz-Odendaal, 2011). This was done in an effort to avoid the potential negative effects of a longer fixation time on staining. Dehydrated specimens were subsequently rehydrated in a reverse graded ethanol series prior to staining. A comparison of specimens that were assayed immediately to those that were dehydrated and rehydrated before being stained revealed that this additional step had no discernable effect on staining (data not shown).

Alkaline Phosphatase (AP) Staining for Osteoblasts: Specimens fixed for 12-24 hours in paraformaldehyde in PBS were washed twice for 5 minutes in protective Tris buffer ( $\mathrm{pH}$ 9.5), then placed in AP detection solution until the stain developed, a length of time which varied depending on fish size and species. The AP detection solution relies on the reaction of AP, 5-bromo-4-chloro-3-indolyl-phosphate (BCIP; 50mg/ml dimethylformamide [DMF], Roche) with nitro blue tetrazolium (NBT; $100 \mathrm{mg} / \mathrm{ml} \mathrm{DMF}$; Roche). BCIP binds to AP, and NBT reacts with the BCIP forming a dark blue insoluble azo dye (Boenisch 2001). Negative controls were done in which DMF (without BCIP) was added in place of the BCIP solution. To determine the ideal staining duration, a doseresponse experiment was conducted. Given that AP could stain just the outermost tissue layers or may stain them more strongly than more interior tissues, a $16 \mathrm{~mm} \mathrm{SL}$ Tramitichromis specimen was bisected prior to staining and sectioned, which confirmed that the stain sufficiently penetrated the tisuse. Whole stained specimens were imaged and then worked up into $80 \%$ glycerol in water for storage prior to histological preparation for detailed analysis in transverse sections. 
Tartrate Resistant Acid Phosphatase (TRAP) Staining for Osteoclasts: After being fixed in 4\% paraformaldehyde in PBS for 12-24 hours, specimens were incubated for 1 hour in tartrate buffer ( $\mathrm{pH} 5.5$ ), followed by a dark incubation for 2 hours in TRAP substrate solution (protocol modified from Edsall and Franz-Odendaal, 2010). The TRAP substrate solution uses the reaction between TRAP, naphthol-AS-TR-phosphate (SigmaAldrich), and hexazotized pararosaniline (PRS). In this reaction, TRAP binds naphtholAS-TR-phosphate, which serves as the substrate for hexazotized PRS. The coupling of naphthol-AS-TR-phosphate and hexazotized PRS labels the TRAP by producing a red azo dye (Boenisch 2001). With each group of fish that went through the TRAP protocol together (in the same solutions), a negative control was performed in which the naphtholAS-TR-phosphate component of the detection solution $(3.3 \%$ of the total volume, added as $1 \mathrm{mg} / \mathrm{ml}$ naphthol-AS-TR-phosphate in DMF) was replaced with DMF (without naphthol-AS-TR-phosphate). TRAP-stained specimens were imaged in brightfield and then worked up into $80 \%$ glycerol in water for storage prior to histological preparation.

Preparation of Histological Material - Samples stained for AP or TRAP and stored in $80 \%$ glycerol were rehydrated through a set of serial glycerol dilutions to distilled water. Eye lenses were removed (to avoid sectioning problems), and individuals $>10 \mathrm{~mm} \mathrm{SL}$ were bissected behind the pectoral fin. Whole fish (or heads of larger individuals) were decalcified in Cal-Ex (Fisher Scientific) on a shaker table for 2 hours (6.0 - 7.5 mm SL), for 3.5 hours $(8.0-8.5 \mathrm{~mm} \mathrm{SL})$, or for 8 hours $(>8.5 \mathrm{~mm} \mathrm{SL})$. Specimens were then dehydrated in a graded ethanol and t-butyl alcohol (Fisher Scientific) series, infiltrated in 
Paraplast (Fisher Scientific) under vacuum, and embedded in Paraplast. Tissues were sectioned transversely at $8 \mu \mathrm{m}$ thickness and all sections were mounted on albuminsubbed slides $(10 \%$ albumin in $0.9 \% \mathrm{NaCl})$. Slides were counterstained with $4 \%$ methyl green in distilled water for 10 minutes, then rapidly dehydrated in absolute ethanol (modified from Presnell and Schreibman 1997, N. Bird, pers. comm.), and coverslipped with Entellan (Electron Microscopy Sciences).

\section{Quantification and Visualization of Osteoblast and Osteoclast Activity Data}

Alkaline phosphatase (AP) staining for osteoblast activity appeared as a diffuse smear of color ranging from light lavender to dark indigo, which was interpreted as corresponding to the intensity of osteoblast activity. In contrast, tartrate-resistant acid phosphatase (TRAP) staining for osteoclasts usually appeared as discrete, red-stained osteoclast cells with darkly stained nuclei, and occasionally as a light wash of red localized to surface of a bone (Figure 9). Thus, different approaches were needed to quantify AP and TRAP staining. For AP staining, a scoring system was devised where the lightest staining (low osteoblast activity) was scored as a 1 and the darkest staining (highest osteoblast activity) was scored as a 4. Although subjective, it was possible to illustrate relative levels of activity, at positions along the canal and around the circumference of the canal. For TRAP staining, a more traditional cell counting method was used since discrete osteoclast cell nuclei were darkly stained and easily identified. Preliminary observations revealed what were apparently multinucleated TRAP-stained osteoclasts, despite the fact that acellular bone in young teleosts is usually associated with

mononucleated osteoclasts (Witten 1997, Witten and Huysseune 2009). In these cases, it 
was unclear whether the cells were multinucleated osteoclasts or closely spaced mononuclear osteoclasts as described by Witten (1997). Since these two alternatives were not reliably distinguishable, TRAP staining intensity (osteoclast activity) was assessed using the total number of TRAP-stained nuclei.

Several data visualization tools were used to visualize AP and TRAP distribution and intensity or abundance around the circumference of the canal, at different positions along the canal length, in fishes of different size classes, in the two study species. After much trial and error, it was determined that the most effective way to represent these data would be to apply a colorimetric scale to a series of pie charts (eg. see Figure 8) where the colors correspond to the relative amount of osteoblast or osteoclast activity as shown by AP and TRAP staining, respectively. In these graphs, a light color represents a low activity level, and a darker color represents a higher activity level. Thus, the pie charts serve as diagrammatic representations of canal cross-sections. For AP data, the mean of the AP scores in each of the quadrants are represented by the AP score in each of the resulting quadrants. For TRAP data, the total number of osteoclast nuclei in each of the quadrants was recorded. The conditional formatting tool in Excel (Microsoft Office v. 15.11.2) was used to generate color gradient scales corresponding to the values of AP (the score of 0-4) and TRAP (the number of osteoclast nuclei counted), with darker colors representing higher scores.

For numerical graphs plotting osteoblast and osteoclast activity across ontogeny, the raw scores (AP) or number of osteoclast nuclei (TRAP) in all four quadrants at all 29 positions in each replicate fish were summed to yield one value that represented the total osteoblast or osteoclast activity in that individual fish. In order for osteoblast and 
osteoclast data to be comparable despite vastly different scales (scores vs. osteoclast nuclei), the maximum total osteoblast or osteoclast activity was identified, and the total activity values were reinterpreted as percentages of that maximum.

\section{Canal Enclosure}

The stage of canal enclosure (Tarby and Webb 2003, Webb and Shirey 2003, Bird and Webb 2014) was also recorded for each histological section analyzed. The resulting data was used to explore patterns in the sequence and timing of canal enclosure in association with each neuromast along the length of the canal.

\section{Statistical Analysis}

Chi-squared $\left(\mathrm{x}^{2}\right)$ tests were computed using $\mathrm{R}$ (version 3.3.2) to test the hypothesis that the osteoclasts (TRAP staining) are uniformly distributed, either along the length of the canal (MD2 to MD4), or among the four quadrants around the circumference of the canal. The subjective nature of the AP data and the lack of an angular value for each TRAP nucleus relative to the axes of the data collection grid precluded other statistical analysis.

\section{RESULTS}

In both Aulonocara and Tramitichromis, vital staining with calcein and 4-di-2ASP revealed five MD canal neuromasts within the developing MD canal (Figure 10). Canal segments formed asynchronously in a given individual. For instance, in a $16 \mathrm{~mm}$ SL fish, the minimum developmental stage observed for MD1 was stage IIb (bony walls, 
but not yet enclosed by epithelium), while the maximum developmental stage for MD3 was stage IV (fully enclosed and ossified).

Aulonocara at $16 \mathrm{~mm}$ SL can be used to illustrate the combination of visualization methods used (Figure 11). MD3 was fully ossified (Stage IV), but MD2 and MD4 segments were enclosed by only epithelium (Stage III), with calcein-stained bone fronts (canal walls) visible extending up and over the neuromast within the soft tissue canal roof. In addition, the positions between MD2 and MD3 ("mid 2-3") and the positions between MD3 and MD4 ("mid 3-4") were the sites of large pores in the roof of the canal. The position of the bone fronts relative to the overlying epithelium in sections confirmed the observations of the developmental stages identified with vital staining. The histological osteoblast and osteoclast assays revealed the activity and distribution of osteoblasts and osteoclasts around the canal circumference at that position along the length of the canal (Figure 11b, c). The radial staining pattern in the MD3 Region revealed light osteoblast activity in the canal roof and under the neuromast, in addition to osteoclast activity under the neuromast and in the canal walls, but not in the canal roof.

\section{Bone Remodeling through Ontogeny in Aulonocara}

The examination of whole fish showed AP staining localized to the edges of the epithelial canal pores in smaller fish and to the edges of the bone fronts (canal walls) within dermal tissue, which were starting to enclose neuromasts in larger fish (e.g., 12 $\mathrm{mm}$ SL; Figure 12). In the smallest size class examined (8 $\mathrm{mm}$ SL fish), background AP staining was present in the surface epithelium, but not in association with the mandibular canal neuromasts. AP staining appeared at the edges of the canal pores in the $12 \mathrm{~mm} \mathrm{SL}$ 
fish, as well as in the canal walls, especially at the articulation between the dentary and anguloarticular bones. In the larger, $16 \mathrm{~mm}$ SL fish, AP staining was visible at the edges of the epithelial canal pores, but also on the edges of the canal walls (bone fronts). In 20 $\mathrm{mm}$ SL fish, AP staining was only associated with the bone fronts, not the epithelial canal pores. In $24 \mathrm{~mm}$ SL fish, the tympanums (where bony pores were bigger than epithelial pores, so epithelium overlaying the wider openings of the bony pores) were visible.

TRAP staining showed a different pattern. In the smallest fish (8 mm SL), TRAP marked two of the five mandibular neuromasts and some tooth buds in the lower jaw (Figure 12). In fish $\geq 12 \mathrm{~mm} \mathrm{SL}$, TRAP was visible in the canal walls (bone fronts). In 16 $\mathrm{mm}$ SL fish, TRAP was also visible in the bony canal roof enclosing the neuromasts. In some cases, there appeared to be more TRAP staining in the medial canal wall than in the lateral canal wall enclosing a neuromast. Unfortunately, TRAP-stained fish showed a high degree of background staining (eg. Figure 12H), which limited further observations of TRAP activity in whole fish.

An analysis of histological material for AP and TRAP staining revealed two peaks in osteoblast activity during ontogeny - one in $12 \mathrm{~mm}$ SL fish and another in $24 \mathrm{~mm} \mathrm{SL}$ fish (Figure 14). Osteoblast activity was found in the canal roof (Quadrant 4) of all Regions along the canal in all five size classes except the smallest one (8 mm SL; Figure 15). The MD2 Region was the only Region showing osteoblast activity in the canal walls (Quadrants 2 and 3) in size classes outside of the two staining peaks. Osteoblast activity was observed behind the neuromast (Quadrant 1) in all Regions during the staining peaks (12 and $24 \mathrm{~mm} \mathrm{SL}$ ), but in other size classes such osteoblast activity was limited to the MD2 and MD3 Regions of the $16 \mathrm{~mm}$ SL animals. In the smallest fish (8 $\mathrm{mm} \mathrm{SL})$ and in 
$20 \mathrm{~mm}$ SL fish there was no evidence of osteoblast activity behind the neuromast (Quadrant 4) in any Region along the canal. The patterns of osteoblast activity were similar in the neuromast-associated and inter-neuromast Regions along the canal in all size classes, except $12 \mathrm{~mm} \mathrm{SL}$. The canal roof (Quadrant 4) was the site of the most osteoblast activity, with low levels around the rest of the canal circumferences, and osteoblast activity across ontogeny peaked at both 12 and $24 \mathrm{~mm} \mathrm{SL}$. However, there was a marked difference between osteoblast activity in neuromast-associated Regions and inter-neuromast Regions along the canal during the $12 \mathrm{~mm} \mathrm{SL}$ activity peak, when all neuromast-associated Regions had osteoblast activity behind the neuromast (Quadrant 1), but no inter-neuromast Regions showed any such activity in this quadrant. Further, with few exceptions, osteoblast activity (bone deposition) was restricted to the interior surface of the canal, contrary to the hypothesis that bone deposition would occur on the exterior surface to maintain canal wall thickness as canal diameter increased.

Osteoclast activity also fluctuated through ontogeny. In smaller animals $(8,12$, and $16 \mathrm{~mm} \mathrm{SL}$ ) there was little activity in any Region along the canal and a peak in activity occurred at $20 \mathrm{~mm}$ SL but then fell to a moderate level in the largest fishes examined (24 mm SL fish; Figure 16). Unlike osteoblast activity, which was usually concentrated in the canal roof (Quadrant 4) and behind (under) the neuromast (Quadrant 1) when present, the site of the most osteoclast activity when present was generally in one or both of the canal walls (Quadrants 2,3). Further, there was little to no osteoclast activity in the canal roof (Quadrant 4) of any of the neuromast-associated Regions along the canal, even in size classes that had very high osteoclast activity levels. The distribution of osteoclast activity around the canal circumference during the peak in 
osteoclast activity (20, $24 \mathrm{~mm}$ SL fish) differs significantly from a uniform distribution (Table 1) in all canal Regions along the canal with two exceptions (MD4 in $20 \mathrm{~mm} \mathrm{SL}$ fish, MD3 and MD4 in $24 \mathrm{~mm}$ SL fish). Osteoclast activity patterns were similar in interneuromast and neuromast-associated Regions along the canal, with peaks in $20-24 \mathrm{~mm}$ SL fish. Canal walls (Quadrants 2, 3) demonstrated more osteoclast activity than either the canal roof (Quadrant 4) or in the bone below the neuromast (Quadrant 1, Figure 16). However, the neuromast-associated Regions had slightly more osteoclast activity in the canal floor (behind the neuromast) than the inter-neuromast Regions.

\section{Bone Remodeling through Ontogeny in Tramitichromis}

As in Aulonocara, AP staining was present in all five size classes of fish, and was associated with the epithelial canal pores in smaller animals $(\leq 16 \mathrm{~mm} \mathrm{SL})$. In $12 \mathrm{~mm} \mathrm{SL}$ fish, AP staining was visible in the bone fronts representing the growing canal walls (Quadrants 2, 3) over each neuromast. In both the 16 and $20 \mathrm{~mm}$ SL fish, AP stained the edges of epithelial pores, but in the largest fish ( $24 \mathrm{~mm} \mathrm{SL})$, staining around the epithelial pore edges was lower, approximately equal to the level of the background staining.

Whole fish stained with TRAP also had high levels of background staining (Figure 12H), but some staining was visible in the tooth buds and some mandibular neuromasts in the smallest fish ( $8 \mathrm{~mm}$ SL; Figure 12F). In the 16 and $20 \mathrm{~mm}$ SL fish, TRAP staining was also apparent in the canal walls and around the epithelial pores of the canals. There was very little canal-associated TRAP staining visible in the $12 \mathrm{~mm} \mathrm{SL}$ and in the largest $(24 \mathrm{~mm})$ fish. 
In Tramitichromis, all Regions along the canal (MD2, mid 2-3, MD3, mid 3-4, and MD4) showed a peak in osteoblast activity in $12 \mathrm{~mm}$ SL fish, with no second peak in the $24 \mathrm{~mm}$ SL fish, which was observed in Aulonocara (Figure 17). The MD3 and MD4 Regions had nearly identical patterns of osteoblast activity (Figure 18). There was activity in all quadrants around the canal circumference, but especially in the canal roof (Quadrant 4), with a peak at $12 \mathrm{~mm}$ SL and a decrease in larger animals. MD2, however, showed a slightly different pattern with no osteoblast activity in the canal roof (Quadrant 4) but activity behind the neuromast (Quadrant 1) before the staining peak (12 mm SL). In larger animals, there was no activity behind the neuromast (Quadrant 1) and activity in the canal roof (Quadrant 4) decreased with size. In the inter-neuromast Regions, the canal roof (Quadrant 4) also showed the most intense osteoblast activity. With one exception (MD2 Region in $12 \mathrm{~mm}$ SL fish), all Regions showed the same overall pattern of osteoblast activity along canal length in each size class. The smallest fish (8 $\mathrm{mm} \mathrm{SL})$ had the strongest activity in the canal floor (Quadrant 1 in neuromast-associated Regions), but in the larger fish, the canal roof (Quadrant 4) was the most darkly stained, with a staining peak in $12 \mathrm{~mm}$ SL fish that decreased in larger fish. As in Aulonocara, osteoblast activity was not observed on the external surface of the canal.

Osteoclast activity peaked in 16 and $20 \mathrm{~mm}$ SL fish and diminished somewhat in the $24 \mathrm{~mm}$ SL fish. During the osteoclast activity peak, several Regions along the canal had non-uniform distributions of osteoclast nuclei around the canal (Figure 19, Table 2). The distribution of osteoclast activity was non-uniform in the MD2 Region in fish $\geq 16$ $\mathrm{mm}$ SL fish, in both inter-neuromast Regions in the 16 and $24 \mathrm{~mm}$ SL fish, and in MD4 in the $20 \mathrm{~mm}$ SL fish (Table 2). Generally, the neuromast-associated Regions in fishes 
$\geq 16 \mathrm{~mm}$ SL showed no osteoclast activity in the canal roof (Quadrant 4), and the highest levels of osteoclast activity in the canal walls (Quadrants 2,3) or floor (Quadrant 1). The MD2 and MD3 Regions had a similar distribution of osteoclast activity, but the MD4 Region was the site of very little osteoclast activity in any size class. This may reflect a rostro-caudal gradient of osteoclast activity, as MD2 was the site of the most osteoclast activity, MD3 showed a similar radial pattern of osteoclast activity but less activity overall, and MD4 showed very little osteoclast activity at all. The inter-neuromast Regions showed a somewhat different radial pattern of osteoclast activity, with little activity in the canal floor (Quadrant 1), and the most activity in the walls (Quadrant 2,3) and canal roof (Quadrant 4) in fish $\geq 16 \mathrm{~mm} \mathrm{SL}$.

\section{"Ring" Staining}

In Aulonocara, A ring-like pattern of AP staining was observed around superficial neuromasts, including presumptive canal neuromasts, prior to enclosure in a canal (Figure 13). In fish $\leq 12 \mathrm{~mm} \mathrm{SL}, \mathrm{AP}$ was visible in the epithelium and in rings around both superficial and canal neuromasts. Though the rings around the surrounding superficial neuromasts were very dark, the rings around the canal neuromasts were faded in $12 \mathrm{~mm}$ SL fish, and were not visible in fish $>16 \mathrm{~mm}$ SL. AP staining in a dark ring around superficial neuromasts was still evident in the largest fishes examined $(24 \mathrm{~mm}$ SL). This ring of AP seemed to disappear as presumptive canal neuromasts were enclosed in the canal, but the staining persisted through ontogeny in neuromasts that remained superficial. 
In Tramitichromis, AP staining was present in a ring pattern around superficial neuromasts in all size classes and presumptive canal neuromasts in fish $<16 \mathrm{~mm}$ SL. In 8 $\mathrm{mm}$ SL fish, all neuromasts were marked by a bulls-eye pattern of AP staining, featuring a darkly stained center and a discrete ring of staining that was darker in superficial neuromasts than presumptive canal neuromasts. Despite heavy background staining, superficial neuromasts were circled by a ring of AP in fishes in all size classes.

\section{An Analysis of MD3 in Detail}

In order to resolve more detailed patterns, a finer scale analysis of the MD3 region was carried out in both species (Figure 20).

In Aulonocara, peaks in osteoblast activity in 12 and $24 \mathrm{~mm}$ SL fish were evident in the vicinity of neuromast MD3 (Positions 17-21) and there was little variation in osteoblast activity among positions along the length of the neuromast (Figure 21). A sizerelated pattern in radial distribution of osteoblast activity was evident. In $12 \mathrm{~mm}$ SL fish there was strong osteoblast activity in the canal roof (Quadrant 4) and behind (under) the neuromast (Quadrant 1). Osteoblast activity appeared to decrease overall in $16 \mathrm{~mm} \mathrm{SL}$ fish, which showed consistent osteoblast activity in the canal roof (Quadrant 4), but inconsistent activity behind the neuromast (Quadrant 1). In the $20 \mathrm{~mm}$ SL fish the only osteoblast activity was in the canal roof (Quadrant 4), but was weaker than in smaller fish (12 and $16 \mathrm{~mm} \mathrm{SL}$ ). In the largest fish (24 mm SL), osteoblast activity was once again very high in the canal roof (Quadrant 4), with little to no activity around the rest of the canal circumference. 
In contrast, a general trend of increasing osteoclast activity with increasing fish size was noted (Figure 22). In $8 \mathrm{~mm}$ SL fish, little TRAP staining was apparent, while in $12 \mathrm{~mm}$ SL fish there was consistently low osteoclast activity, but the radial distribution of this activity was variable among positions along the neuromast. The $16 \mathrm{~mm} \mathrm{SL} \mathrm{fish}$ showed a more consistent pattern of moderate levels of osteoclast activity under the neuromast (Quadrant 1). The $20 \mathrm{~mm}$ SL fish showed a peak in osteoclast activity, with the highest level of osteoclast activity overall, concentrated primarily in the canal walls (Quadrant 2, 3), with none in the canal roof (Quadrant 4) in all but the most rostral position (Position 17). In the largest fish (24 mm SL), TRAP staining was concentrated in the canal walls (Quadrants 2,3) near the center of the neuromast (positions $18-20$ ), and included moderate staining in the canal roof (Quadrant 4) at the rostral and caudal edges of the neuromast (positions 17,21). This may be explained by the fact that positions near the edges of the neuromast are in closer proximity to the canal pores on either side.

In Tramitichromis, in each size class, osteoblast activity was consistent among positions along the neuromast (Figure 23). Generally, there was one peak in osteoblast activity in $12 \mathrm{~mm}$ SL fish that then decreased in larger fish. The $8 \mathrm{~mm}$ SL fish showed moderate staining below the neuromast (Quadrant 1) along its length (Positions 17 - 21). In $12 \mathrm{~mm}$ SL fish there was strong staining in the canal roof (Quadrant 4) and moderate staining around the rest of the canal circumference, but the $16 \mathrm{~mm}$ fish showed little staining in the canal roof. The $20 \mathrm{~mm}$ SL fish showed even less staining in the canal roof, and Position 18 had no staining in any quadrant. The $24 \mathrm{~mm}$ SL fish also showed no AP staining in any of the quadrants around the canal circumference. 
The pattern of osteoclast activity was more variable than that of osteoblasts. However, osteoclast activity showed a more consistent pattern in Tramitichromis than it did in Aulonocara (Figure 24). As in Aulonocara, there appeared to be a staining peak in 16 and $20 \mathrm{~mm}$ SL fish, with the majority of osteoclast activity concentrated under the neuromast (Quadrant 1) and in the canal walls (Quadrants 2, 3). In the two smallest size classes ( 8 and $12 \mathrm{~mm} \mathrm{SL}$ ) fish showed very little osteoclast activity, with a low level of activity under the neuromast (Quadrant 1). Larger fish (16 and $20 \mathrm{~mm} \mathrm{SL}$ ) showed moderate levels of osteoclast activity under the neuromast (Quadrant 1) and in the medial canal wall (Quadrant 2). The largest fish (24 mm SL) had very little osteoclast activity in any location along the length of the neuromast.

In both Aulonocara and Tramitichromis, there did not appear to be any spatial pattern of canal enclosure, for example, rostral positions enclosing first, within a given neuromast (Figures 25 and 26).

\section{DISCUSSION}

This study used histochemical methods to determine the roles of bone-forming (osteoblasts) and bone-resorbing (osteoclasts) cells in the initial morphogenesis of the lateral line canals, the growth of the canals (increases in diameter), and the generation of narrow and widened canal phenotypes during ontogeny. In both study species, Aulonocara stuartgranti (widened canals) and Tramitichromis sp. (narrow canals), osteoblast activity was concentrated in the roof and floor of the canal, while osteoclast activity was highest in the lateral canal walls that extend to enclose the neuromasts. Another similarity between the two species was the presence of two pulses of bone cell 
activity during the time period sampled: one early pulse defined by bone deposition in both species, and later in development, a second pulse of either bone resorption (Tramitichromis) or bone remodeling (Aulonocara). The patterns and timing of osteoblast and osteoclast activity revealed by this study corroborate the observations by Bird and Webb (2014), that dissociated heterochrony, that is, differences in developmental timing, is a major factor in the morphogenesis of divergent lateral line canal phenotypes. Further, in the context of neuromast-centered canal morphogenesis, this study suggests that the influence of neuromast proximity on the spatial pattern of canal morphogenesis at the cell level may vary depending on ultimate adult canal phenotype.

\section{Osteoblast and Osteoclast Activity during Initial Canal Morphogenesis}

This study showed that osteoblasts and osteoclasts are present in mid- to latestage larvae and early juveniles ( $8-24 \mathrm{~mm} \mathrm{SL}$ ) in both Aulonocara and Tramitichromis, but that the intensity and pattern of the activity of these two cell types vary in space and in ontogenetic time in each species. The total amount of osteoblast and osteoclast activity was generally consistent along the length of the canal, but each was non-uniformly distributed around the canal circumference, and osteoblasts and osteoclasts demonstrated largely non-overlapping distributions. In fish of all size classes examined, where osteoblasts or osteoclasts were present, osteoblasts were most active in the roof (Quadrant 4) and floor of the canal (Quadrant 1), while osteoclasts were most active in the canal walls (Quadrants 2 and 3).

While both osteoblasts and osteoclasts were present in the larvae and early juveniles examined ( $8-24 \mathrm{~mm} \mathrm{SL})$, there were two distinct time periods of marked high 
activity in both species (Figure 27). An early peak in osteoblast activity occurred at $8-12$ $\mathrm{mm}$ SL (with low levels of osteoclast activity), followed by a peak in osteoclast activity (20-24 mm SL). Between these two peaks (e.g., in fishes of $16 \mathrm{~mm} \mathrm{SL}$ ), both osteoblast and osteoclast activity were relatively low.

The first peak in osteoblast activity (8-12 mm SL) suggests intense bone deposition, but some differences were observed between species. Tramitichromis had higher levels of osteoblast activity than Aulonocara, and the first peak in osteoblast activity in Tramitichromis occurred in smaller fishes (8 $\mathrm{mm} \mathrm{SL})$ than in Aulonocara (12 $\mathrm{mm}$ SL). Similarly, there were differences in timing of the second peak in osteoclast activity between species. Additionally, In Tramitichromis, the second peak was defined by high osteoclast activity, but little to no osteoblast activity. Thus, the MD canal in Tramitichromis appears to develop as the result of one pulse of bone deposition and a subsequent pulse of bone resorption. By contrast, after the first peak of bone deposition in Aulonocara, the second peak is defined by high levels of both osteoclast and osteoblast activity. Thus, the process of canal morphogenesis in Aulonocara appears to occur as a result of a pulse of bone deposition followed by a pulse of simultaneous osteoblast and osteoclast activity (active bone remodeling). It is concluded that the generation of the narrow and widened canal phenotypes is the result of these differences in the spatial pattern and timing of osteoblast and osteoclast activity.

\section{Osteoblasts and Osteoclast Activity in Canal Growth}

The spatial and ontogenetic distributions of osteoblasts and osteoclasts can also be used to understand how the canal diameter increases, a process that continues after initial 
morphogenesis in concert with fish growth (Bird and Webb, 2014). In both species, the early pulse of osteoblast activity is followed by high levels of osteoclast activity. This suggests a general theme of canal growth by alternating periods of bone deposition and resorption or remodeling resulting in the continuous expansion of canal diameter. This was noted in Aulonocara and Tramitichromis by Bird and Webb (2014), in zebrafish by Moore (2008), and in cichlids by Tarby and Webb (2003), and thus appears to be a generalized pattern of canal development. In both Aulonocara and Tramitichromis, osteoclast activity is low in early larval ontogeny $(<16 \mathrm{~mm} \mathrm{SL})$ but is higher in larger larval and juvenile fish (16-24 $\mathrm{mm} \mathrm{SL})$. This can account for the increase in canal diameter in these species reported by Bird and Webb (2014).

In Tramitichromis, despite low levels of osteoclast activity throughout ontogeny relative to Aulonocara, there are more active osteoclasts in larger animals $(16-20 \mathrm{~mm}$ SL) than in smaller animals $(<16 \mathrm{~mm} \mathrm{SL})$. These osteoclasts presumably increase the diameter of the canal lumen by removing bony matrix on the internal surface of the canal. In Aulonocara, the level of osteoclast activity (indicated by the numbers of osteoclasts present) is also increased in larger individuals (compared to smaller ones), and these high levels of osteoclast activity on the internal surface coincide with a second pulse of osteoblast activity on the internal surface of the canals.

These observations support the idea that in Tramitichromis, canals are formed by a simple pattern: a pulse of bone deposition (at 8-12 $\mathrm{mm} \mathrm{SL}$ ) followed by bone resorption which begins at a low levels and increases as the fish grows, peaking in larger fish (20-24 mm SL). In contrast, in Aulonocara, there is more osteoclast activity overall through time, perhaps because bone resorption is necessary to continuously enlarge the (widened) 
canal lumen while actively remodeling the new bone that osteoblasts are laying down. Thus, increased osteoclast activity in Aulonocara shows that canal morphogenesis in Aulonocara is a sequence of bone deposition and bone remodeling (bone deposition and resorption, as opposed to only resorption, as in Tramitichromis). The fish studied only represent a snapshot of canal enlargement during a limited size interval ( $8-24 \mathrm{~mm}$ SL) in larvae and young juveniles, so it is possible that patterns of alternating bone deposition by osteoblasts and bone resorption by osteoclasts (in Tramitichromis), or bone deposition by osteoblasts and bone remodeling by simultaneous activity of osteoblasts and osteoclasts (in Aulonocara), are repeated in even larger fishes (juveniles and adults). In the case of Aulonocara, another possibility is that the high osteoblast and osteoclast activity levels in the largest fish studied $(20-24 \mathrm{~mm} \mathrm{SL})$ persist, constantly remodeling the canal as the fish continues to grow.

\section{Evidence of Neuromast-Centered Canal Morphogenesis at the Cell Level}

Neuromast-centered canal morphogenesis is the idea, supported by the observations of several authors (reviewed in Webb 2014b) that canals tend to start to form in the vicinity of individual neuromasts. This suggests that neuromasts are somehow involved in the process of canal morphogenesis, potentially in some instructive or inductive capacity (J. Webb, pers. comm.; discussed in Hall 2015). It had been predicted that differences in either the intensity, ontogenetic timing, or radial distribution of osteoblasts or osteoclasts would differ in the vicinity of canal neuromasts (neuromastassociated regions) versus locations between canal neuromasts (inter-neuromast regions). 
However, the results of this study showed that osteoblast and osteoclast activity in neuromast-associated versus inter-neuromast regions were more similar than expected. In Tramitichromis, in particular, both neuromast-associated and inter-neuromast regions had similar osteoblast and osteoclast activities (Figure 28b). By contrast, in Aulonocara, the first pulse of osteoblast activity $(12 \mathrm{~mm} \mathrm{SL})$ had a different radial distribution in neuromast-associated regions versus inter-neuromast regions along the canal (Figure 28a). Specifically, all neuromast-associated regions had osteoblast activity in the canal floor (Quadrant 1), while inter-neuromast regions had none (Figure 28a). Thus, the consistency in the distribution of osteoblast activity between neuromastassociated and inter-neuromast regions along the canal in Tramitichromis was in stark contrast to the variability in the distribution of osteoblast activity in Aulonocara (Figure 29). These data are consistent with the observation that widened canals (Aulonocara) form as a series of scallops, or oscillations in the height of the canal walls along the canal during canal morphogenesis, with a maximum height in the vicinity of individual neuromasts. Thus, the results of this study suggest that narrow canals in Tramitichromis form as more continuous trenches with more uniform canal walls in contrast to the "scallops" observed along the widened canals of Aulonocara, where the canals wall are more prominent at neuromast positions.

\section{CONCLUSIONS}

As the first study to assay not just osteoclast activity, but both osteoblast and osteoclast activity in the cranial lateral line canals of fishes, this investigation revealed key variation in osteoblast and osteoclast activity through ontogeny. In both species, 
osteoblast activity (bone deposition) was concentrated in the canal floor and roof, while osteoclast activity (bone resorption) was focused in the canal walls. There was a period of bone deposition early in development, followed by a period of bone resorption (in Tramitichromis) or bone remodeling (bone deposition and resorption, in Aulonocara). The development of Tramitichromis canals is marked by a strong, early pulse of bone deposition and a subsequent moderate pulse of bone resorption, while Aulonocara canals are formed by a less intense pulse of bone deposition, slightly delayed relative to Tramitichromis, and followed by a period of active bone remodeling. These observations provide cell-level evidence of bone development through ontogeny that fits the pattern of dissociated heterochrony (described by Bird and Webb 2014) that underlies the divergence of canal phenotype in these two species.

Considering the phylogenetic distribution of cranial lateral line canal phenotypes among bony fishes (Webb, 2014b), the results obtained here could lead to a new set of questions about the nature of canal formation among taxa. These might include investigations into whether developmental pattern is conserved among taxa with the same canal phenotype - i.e. are all narrow canals formed the same way? If there is a conserved developmental pattern that defines canal phenotype, which canal phenotypes are more similar to each other - how does the development of narrow-simple canals compare to that of reduced canals among distantly related taxa? If there is not a conserved developmental pattern for each canal phenotype, what variables do influence canal development? Could canal development be plastic?

Future studies should look more closely at early ontogeny using in situ hybridization techniques in combination with histochemical methods to more precisely 
define the spatial distributions of osteoblasts and osteoclasts and their gene products.

Additional experiments involving pulses of calcein or other stains throughout ontogeny and during initial canal enclosure would provide insight regarding differences in patterns of canal ossification (calcium deposition) and how these patterns contribute to phenotypic differences in adults. 


\section{LITERATURE CITED}

Albertson RC, Streelman JT, Kocher TD, Yelick PC. 2005. Integration and evolution of the cichlid mandible: the molecular basis of alternate feeding strategies. Proc Natl Acad Sci 102:16287-16292.

Allender CJ, Seehausen O, Knight ME, Turner GF, Maclean N. 2003. Divergent selection during speciation of Lake Malawi cichlid fishes inferred from parallel radiations in nuptial coloration. Proc Natl Acad Sci 100:14074-14079.

Becker EA, Bird NC, and Webb, JF 2016. Post-embryonic development of canal and superficial neuromasts and the generation of two cranial lateral line phenotypes. $\mathrm{J}$ Morphol 277:1273-1291.

Bird NC, Webb JF. 2014. Heterochrony, modularity, and the functional evolution of the mechanosensory lateral line canal system of fishes. EvoDevo 5:21.

Blaxter JHS. 1987. Structure and development of the Lateral Line. Biol Rev 62:471-514.

Boenisch T. 2001. Basic Enzymology. In Handbook of Immunochemical Staining Methods, $3^{\text {rd }}$ Edition (Ed. T Boenisch), pp. 14-17. Dako Corporation, CA.

Chitnis AB, Dalle Nogare D, Matsuda M. 2012. Building the posterior lateral line system in zebrafish. Dev Neurobiol 72:234-255.

Coombs S, Janssen J, Webb JF. 1988. Diversity of lateral line systems: evolutionary and functional considerations. In: Sensory Biology of Aquatic Animals. New York, NY: Springer New York. pp. 553- 593.

Coombs S, Janssen J, Montgomery J. 1992. Functional and evolutionary implications of peripheral diversity in lateral line systems. In: The Evolutionary Biology of Hearing (ed. D. B. Webster, R. R. Fay and A. N. Popper). New York, NY: Springer New York. pp. 267- 294.

Cooper WJ, Wernle J, Mann K, Albertson RC. 2011. Functional and genetic integration in the skulls of Lake Malawi cichlids. Evol Biol 38:316-334.

Cruz IA, Kappedal R, Mackenzie SM, Hailey DW, Hoffman TL, Schilling TF, Raible DW. 2015. Robust regeneration of adult zebrafish lateral line hair cells reflects continued precursor pool maintenance. Dev Biol 402:229-238.

Cubbage CC, Mabee PM. 1996. Development of the cranium and paired fins in the zebrafish Danio rerio (Ostariophysi, Cyprinidae). J Morphol 229:121-160. 
Dambly-Chaudière C, Sapède D, Soubiran F, Decorde K, Gompel N, Ghysen A. 2003. The lateral line of zebrafish: A model system for the analysis of morphogenesis and neural development in vertebrates. Biol Cell 95:579-587.

Denton EJ, Gray JAB. 1988. Mechanical factors in the excitation of the lateral lines of fishes. In: Sensory Biology of Aquatic Animals New York, NY: Springer New York. p. 595-617.

Dijkgraaf S. 1963. The functioning and significance of the lateral-line organs. Biol Rev 38:51-105.

Edsall SC, Franz-Odendaal TA. 2010. A quick whole-mount staining protocol for bone deposition and resorption. Zebrafish 7:275-280.

Ekanayake S, Hall BK. 1988. Ultrastructure of the osteogenesis of acellular vertebral bone in the Japanese medaka Oryzias latipes (Teleostei, Cyprinidontidae). Am J Anat 182:241-249.

Franz-Odendaal TA, Ryan K, Hall BK. 2007. Developmental and morphological variation in the teleost craniofacial skeleton reveals an unusual mode of ossification. J Exp Zool Part B Mol Dev Evol 308B:709-721.

Fryer G, Iles TD. 1972. Cichlid Fishes of the Great Lakes of Africa. Oliver \& Boyd.

Fujimura K, Okada N. 2008. Bone development in the jaw of Nile tilapia Oreochromis niloticus (Pisces: Cichlidae). Dev Growth Differ 50:339-355.

Ghysen A, Dambly-Chaudière C. 2004. Development of the zebrafish lateral line. Curr Opin Neurobiol 14: 67-73.

Gompel N, Cubedo N, Thisse C, Thisse B, Dambly-Chaudière C, Ghysen A. 2001. Pattern formation in the lateral line of zebrafish. Mech Dev 105:69-77.

Hall BK. 2015. Bones and Cartilage. London, UK: Academic Press. 920 p.

Huysseune A. 2000. The skeletal system. In The Handbook of Experimental Animals: The Laboratory Fish (Ed. GK Ostrander), pp. 307-319. Academic Press, CA.

Janssen J. 1997. Comparison of response distance to prey via the lateral line in the ruffe and yellow perch. J Fish Biol 51:921-930.

Klein A, Bleckmann H. 2015. Function of lateral line canal morphology. Integr Zool $10: 111-121$.

Kocher TD. 2004. Adaptive evolution and explosive speciation: the cichlid fish model. Nat Rev Genet 5:288-298. 
Konings A. 1990. Koning's Book of Cichlids and Other Fishes of Lake Malawi. TFH Publications Inc., Neptune City, New Jersey

Konings A. 2007. Malawi Cichlids in Their Natural Habitat, 4th ed. Cichlid Press, El Paso, Texas

Ledent V. 2002. Postembryonic development of the posterior lateral line in zebrafish. Dev 129:597-604.

López-Schier H, Starr CJ, Kappler JA, Kollmar R, Hudspeth AJ. 2004. Directional cell migration establishes the axes of planar polarity in the posterior lateral-Line organ of the zebrafish. Dev Cell 7:401-412.

Ma EY, Raible DW. 2009. Signaling pathways regulating zebrafish lateral line development. Curr Biol 19:R381-R386.

McHenry MJ, Liao JC. 2013. The hydrodynamics of flow stimuli. In: The Lateral Line System (Eds. S. Coombs, H. Bleckmann, R.R. Fay, and A.N. Popper). New York, NY: Springer New York. p. 73-98.

Moss ML. 2006. The biology of acellular teleost fish bone. Ann N Y Acad Sci 109:337350.

Münz H. 1979. Morphology and innervation of the lateral line system in Sarotherodon niloticus (L.) (Cichlidae, Teleostei). Zoomorphologie 93:73- 86.

Nemoto Y, Higuchi K, Baba O, Kudo A, Takano Y. 2007. Multinucleate osteoclasts in medaka as evidence of active bone remodeling. Bone 40:399-408.

Northcutt RG, Gans C. 1983. The genesis of neural crest and epidermal placodes: a reinterpretation of vertebrate origins. Q Rev Biol 58:1-28.

Parsons KJ, Marquez E, Albertson RC. 2012. Constraint and opportunity: the genetic basis and evolution of modularity in the cichlid mandible. Am Nat 179:64-78.

Powder KE, Milch K, Asselin G, Albertson RC. 2015. Constraint and diversification of developmental trajectories in cichlid facial morphologies. Evodevo 6:25.

Presnell JK, Schreibman MP. 1997. Humason's Animal Tissue Techniques. Baltimore, MD: Johns Hopkins University Press. 572 p.

Raible DW, Kruse GJ. 2000. Organization of the lateral line system in embryonic zebrafish. J Comp Neurol 421:189-198. 
Romero-Carvajal A, Navajas Acedo J, Jiang L, Kozlovskaja-Gumbriene A, Alexander R, Li H, Piotrowski T. 2015. Regeneration of sensory hair cells requires localized interactions between the notch and wnt pathways. Dev Cell 34:267-282.

Sapède D, Gompel N, Dambly-Chaudière C, Ghysen A. 2002. Cell migration in the postembryonic development of the fish lateral line. Development 129:605-615.

Schleuter D, Eckmann R. 2006. Competition between perch (Perca fluviatilis) and ruffe (Gymnocephalus cernuus): The advantage of turning night into day. Freshw Biol 51:287-297.

Schwalbe MAB, Bassett DK, Webb JF. 2012. Feeding in the dark: lateral-line-mediated prey detection in the peacock cichlid Aulonocara stuartgranti. J Exp Biol 215:20602071.

Schwalbe MAB, Webb JF. 2013. Sensory basis for detection of benthic prey in two Lake Malawi cichlids. Zoology. 117:112-121.

Schwalbe MAB, Webb JF. 2015. The effect of light intensity on prey detection behavior in two Lake Malawi cichlids, Aulonocara stuartgranti and Tramitichromis sp. J Comp Physiol A Neuroethol Sensory, Neural, Behav Physiol 201:341-356.

Schwalbe MAB, Sevey B, Webb JF. 2016. Detection of artificial water flows by the lateral line system of a benthic feeding cichlid fish. J Exper Biol. 219:1050-1059

Seehausen O, Terai Y, Magalhaes I, Carleton K, Mrosso H, Miyagi R, van der Sluijs I, Schneider M, Maan M, Tachida H, Imai H, Okada N. 2008. Speciation through sensory drive in cichlid fish. Nature 455:620-627.

Tarby ML, Webb JF. 2003. Development of the supraorbital and mandibular lateral line canals in the cichlid, Archocentrus nigrofasciatus. J Morphol 255:44-57.

Thomas ED, Cruz IA, Hailey DW, Raible DW. 2015. There and back again: development and regeneration of the zebrafish lateral line system. Wiley Interdiscip Rev Dev Biol 4: $1-16$.

van Snick Gray E, Stauffer J. 2004. Phenotypic plasticity: its role in trophic radiation and explosive speciation in cichlids (Teleostei: Cichlidae). Anim Biol 54:137-158.

Van Trump WJ, McHenry MJ. 2008. The morphology and mechanical sensitivity of lateral line receptors in zebrafish larvae (Danio rerio). J Exp Biol 211:2105-2115.

Wada H, Ghysen A, Satou C, Higashijima S ichi, Kawakami K, Hamaguchi S, Sakaizumi M. 2010. Dermal morphogenesis controls lateral line patterning during postembryonic development of teleost fish. Dev Biol 340:583-594. 
Wada H, Iwasaki M, Kawakami K. 2014. Development of the lateral line canal system through a bone remodeling process in zebrafish. Dev Biol 392:1-14.

Webb JF. 1989. Gross Morphology and Evolution of the Mechanoreceptive Lateral-Line System in Teleost Fishes (Part 1 of 2). Brain Behav Evol 33:34-53.

Webb JF. 2014a. Morphological Diversity, Development, and Evolution of the Mechanosensory Lateral Line System. In: The Lateral Line System (Eds. S. Coombs, H. Bleckmann, R.R. Fay, and A.N. Popper). New York, NY: Springer New York. p. 17-72.

Webb JF. 2014b. Lateral line morphology and development and implications for the functional ontogeny of flow sensing of fishes. In: Flow Sensing in Air and Water Behavioural, Neural and Engineering Principles of Operation. p. 247-270.

Webb JF, Bird NC, Carter L, Dickson J. 2014. Comparative development and evolution of two lateral line phenotypes in Lake Malawi cichlids. J Morpho275:678-692.

Webb JF, Montgomery JC, Mogdans J. 2008. Bioacoustics and the Lateral Line System of Fishes. In: Fish Bioacoustics New York, NY: Springer New York. p. 145-182.

Webb JF, Shirey JE. 2003. Postembryonic Development of the Cranial Lateral Line Canals and Neuromasts in Zebrafish. Dev Dyn 228:370-385.

Weigele J, Franz-Odendaal TA. 2016. Functional bone histology of zebrafish reveals two types of endochondral ossification, different types of osteoblast clusters and a new bone type. J Anat 229:92-103.

Weiss RE, Watabe N. 1979. Studies on the biology of fish bone. III. Ultrastructure of osteogenesis and resorption in osteocytic (cellular) and anosteocytic (acellular) bones. Calcif Tissue Int 28:43-56.

Windsor SP, McHenry MJ. 2009. The influence of viscous hydrodynamics on the fish lateral-line system. Integr Comp Biol 49:691-701.

Witten PE, Villwock W. 1997. Growth requires bone resorption at particular skeletal elements in a teleost fish with acellular bone (Oreochromis niloticus, Teleostei: Cichlidae). J Appl Ichthyol 13:149-158.

Witten PE. 1997. Enzyme histochemical characteristics of osteoblasts and mononucleated osteoclasts in a teleost fish with acellular bone (Oreochromis niloticus, Cichlidae). Cell Tissue Res 287:591-599.

Witten PE, Hansen A, Hall BK. 2001. Features of mono- and multinucleated bone resorbing cells of the zebrafish Danio rerio and their contribution to skeletal development, remodeling, and growth. J Morphol 250:197-207. 
Witten PE, Huysseune A. 2009. A comparative view on mechanisms and functions of skeletal remodeling in teleost fish, with special emphasis on osteoclasts and their function. Biol Rev 84:315-346.

Yanase K, Herbert NA, Montgomery JC. 2014. Unilateral ablation of trunk superficial neuromasts increases directional instability during steady swimming in the yellowtail kingfish Seriola lalandi. J Fish Biol 85: 838-856. 
Table 1. Results of Chi Square Tests of Uniform Radial Distribution of Osteoclast Nuclei in Aulonocara stuartgranti. $\mathrm{n} / \mathrm{a}=$ test could not be performed because too many of the values to be tested were zero. $\mathrm{ns}=\mathrm{p}$-value was greater than 0.05 : non-significant. $*=\mathrm{p}$ value was less than 0.05 : significant.

\begin{tabular}{|c|c|c|}
\hline TEST & P-VALUE & SIGNIFICANCE \\
\hline \multicolumn{3}{|l|}{$8 \mathrm{~mm} \mathrm{SL}$} \\
\hline MD2 & $\mathrm{n} / \mathrm{a}$ & -- \\
\hline $\operatorname{mid} 2-3$ & $\mathrm{n} / \mathrm{a}$ & -- \\
\hline MD3 & $\mathrm{n} / \mathrm{a}$ & -- \\
\hline $\operatorname{mid} 3-4$ & $\mathrm{n} / \mathrm{a}$ & -- \\
\hline MD4 & $\mathrm{n} / \mathrm{a}$ & -- \\
\hline \multicolumn{3}{|l|}{$12 \mathrm{~mm} \mathrm{SL}$} \\
\hline MD2 & 0.881 & ns \\
\hline $\operatorname{mid} 2-3$ & 0.05467 & ns \\
\hline MD3 & 0.9627 & ns \\
\hline mid 3-4 & 0.9841 & ns \\
\hline MD4 & 0.5319 & ns \\
\hline \multicolumn{3}{|l|}{$16 \mathrm{~mm} \mathrm{SL}$} \\
\hline MD2 & 0.129 & ns \\
\hline $\operatorname{mid} 2-3$ & 0.6149 & ns \\
\hline MD3 & 0.0875 & ns \\
\hline mid 3-4 & 0.5724 & ns \\
\hline MD4 & 0.1447 & ns \\
\hline \multicolumn{3}{|l|}{$20 \mathrm{~mm} \mathrm{SL}$} \\
\hline MD2 & 0.000487 & $*$ \\
\hline $\operatorname{mid} 2-3$ & 0.02929 & $*$ \\
\hline MD3 & 0.0549 & ns \\
\hline $\operatorname{mid} 3-4$ & 0.00489 & $*$ \\
\hline MD4 & 0.08966 & ns \\
\hline \multicolumn{3}{|l|}{$24 \mathrm{~mm} \mathrm{SL}$} \\
\hline MD2 & $4.63 \mathrm{E}-06$ & $*$ \\
\hline $\operatorname{mid} 2-3$ & 0.02675 & $*$ \\
\hline MD3 & 0.004956 & $*$ \\
\hline mid 3-4 & 0.000832 & $*$ \\
\hline MD4 & 0.3916 & $\mathrm{~ns}$ \\
\hline
\end{tabular}


Table 2. Results of Chi square Test for Uniform Radial Distribution of Osteoclast Nuclei in Tramitichromis. $\mathrm{n} / \mathrm{a}=$ test could not be performed because too many of the values to be tested were zero. $n s=p$-value was greater than 0.05 : non-significant. $*=p$-value was less than 0.05: significant.

\begin{tabular}{ccc}
\multicolumn{1}{c}{ Test } & p-value & Significance \\
\hline $8 \mathrm{~mm} \mathrm{SL}$ & 0.3916 & $\mathrm{~ns}$ \\
MD2 & $\mathrm{n} / \mathrm{a}$ & -- \\
mid 2-3 & $\mathrm{n} / \mathrm{a}$ & -- \\
MD3 & $\mathrm{n} / \mathrm{a}$ & -- \\
$\operatorname{mid} 3-4$ & $\mathrm{n} / \mathrm{a}$ & -- \\
MD4 & &
\end{tabular}

$12 \mathrm{~mm} \mathrm{SL}$

MD2

mid 2-3

$\mathrm{n} / \mathrm{a}$

MD3

mid 3-4

MD4

0.1116

ns

0.3916

ns

$\mathrm{n} / \mathrm{a}$

$\mathrm{n} / \mathrm{a}$

$16 \mathrm{~mm} \mathrm{SL}$

\section{MD2}

mid 2-3

9.54E-06

0.0231

0.4415

MD3

mid 3-4

MD4

0.001817

$\mathrm{n} / \mathrm{a}$

$20 \mathrm{~mm} \mathrm{SL}$

MD2

2.67E-07

0.1116

0.08332

0.06777

mid 3-4

0.02929

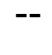

MD4

0.000577

0.03305

0.2929

0.007651

mid 3-4

0.007383 

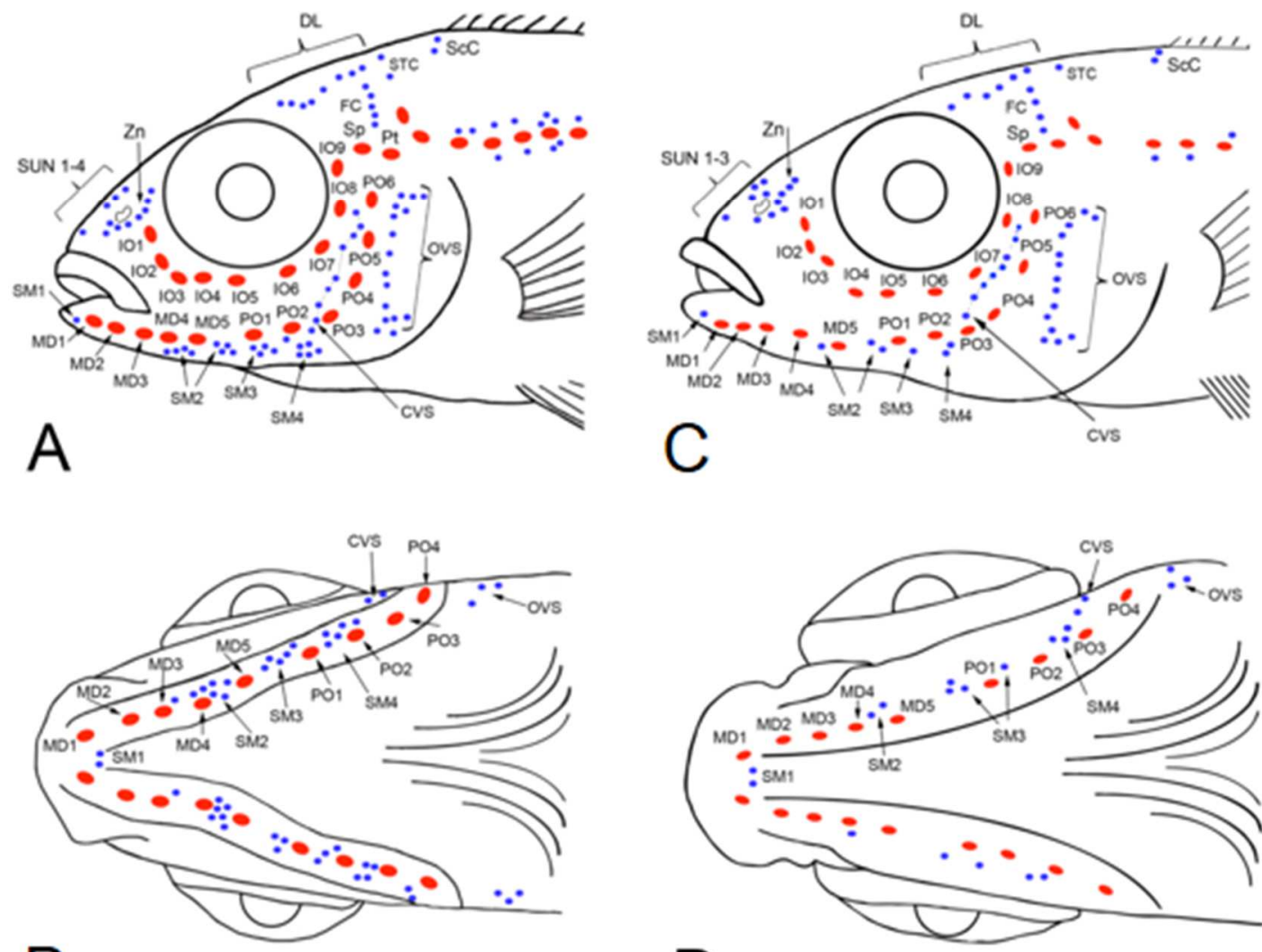

B

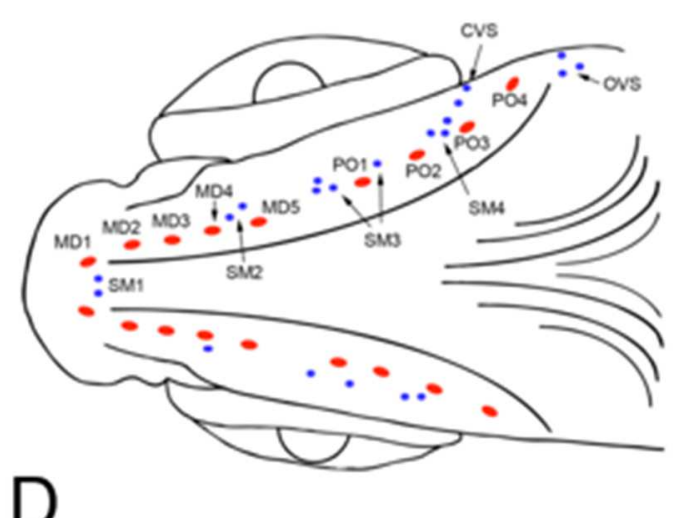

Figure 1. Distribution of neuromasts on the head of Aulonocara stuartgranti and Tramitichromis. Canal and superficial neuromast names and distributions in dorsal, lateral views in (A, B) Aulonocara stuartgranti and (C,D) Tramitichromis sp. Canal neuromasts are represented by large red dots/ovals and superficial neuromasts are represented by the smaller blue dots. Mandibular canal neuromasts are denoted MD1-MD5. Modified from Becker et al. 2016. 


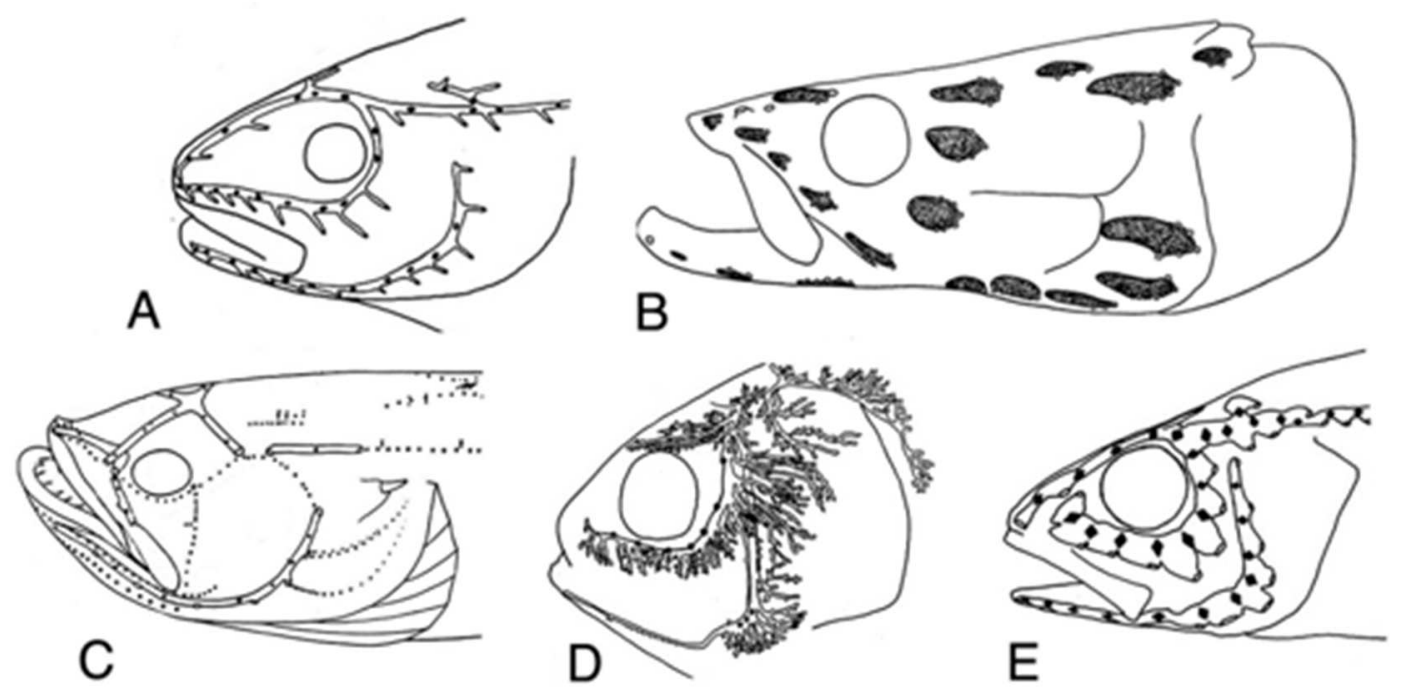

Figure 2. Five patterns of cranial canal systems found among teleost fishes. (a) Narrow-simple canal system (saithe, Pollachius virens). (b) Narrow canals with widened tubules (Arapaima). (c) Reduced canal system with lines of superficial neuromasts (dots) in the plainfin midshipman (Porichthys notatus). (d) Narrow with branched canal system (Atlantic menhaden, Brevoortia tyrannus). (e) Widened canal system in common pecarina (Percarina demidoffi). From Webb 2014 b. 

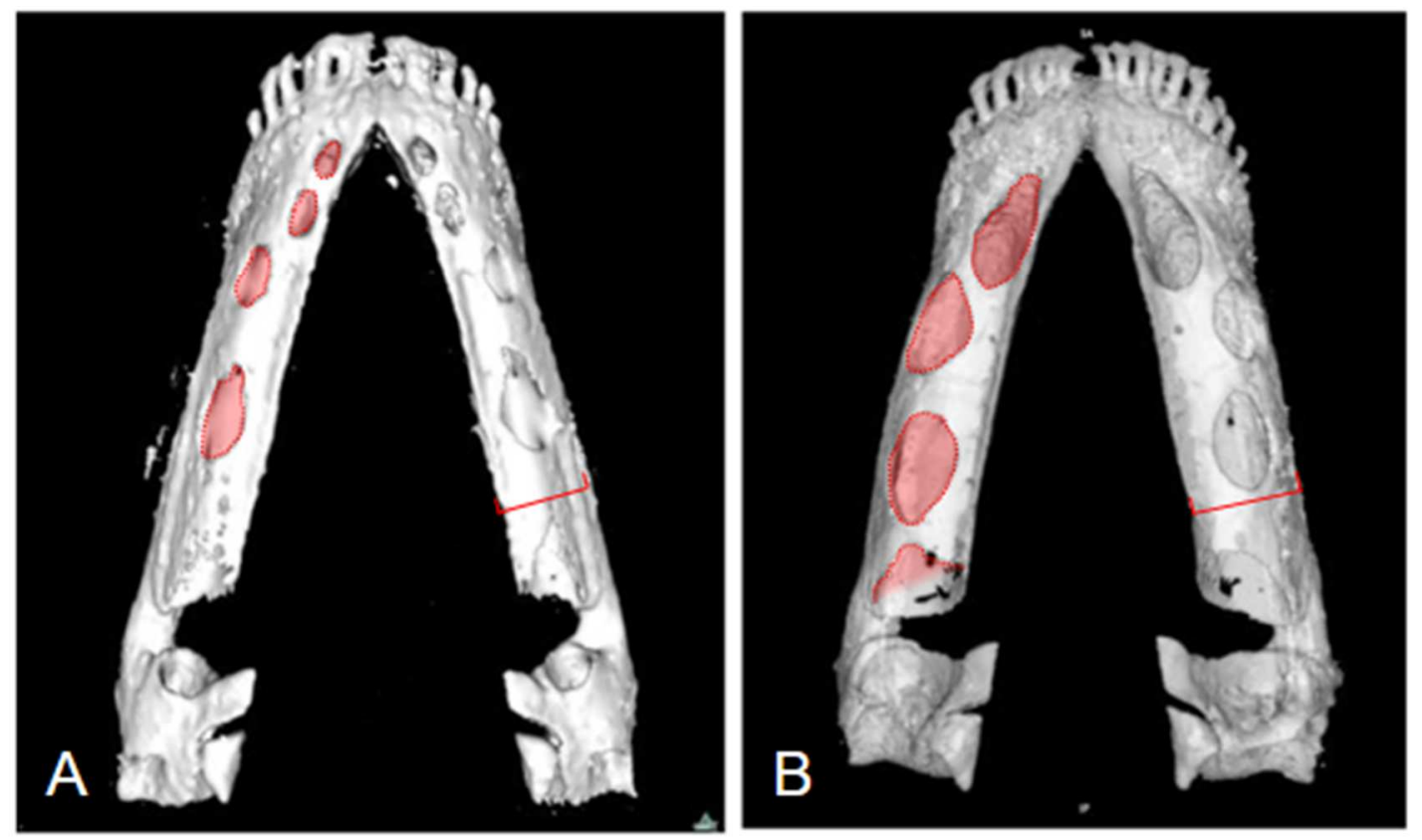

Figure 3. Widened and narrow canals in the mandible of Tramitichromis sp. and Aulonocara stuartgranti. Three-dimensional reconstructions of the mandible (ventral view) from CT scans (16 $\mu$ m slice thickness) of A) Tramitichromis sp. (narrow canals) and B) Aulonocara stuartgranti (widened canals). Note significantly larger canal pores in Aulonocara. Modified from Schwalbe and Webb 2013. 

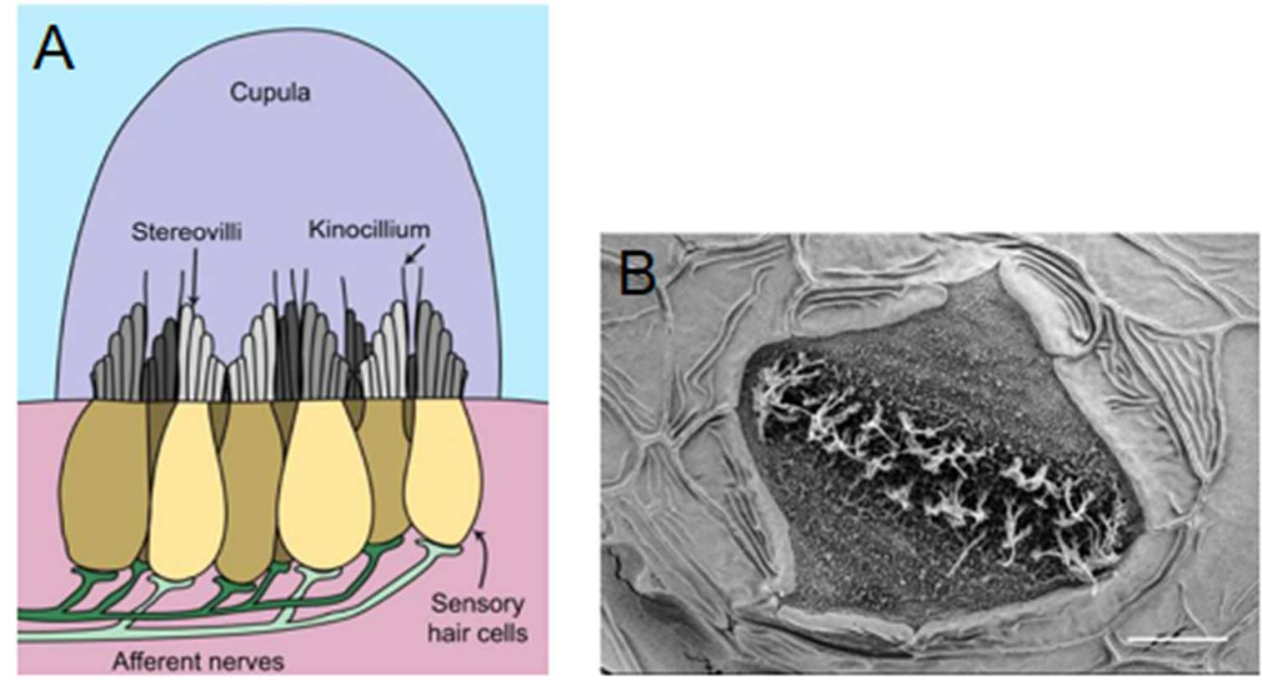

Figure 4. Anatomy of a neuromast. A) Diagram of neuromast structure showing support cells and kinocilium and graded stereocilia projecting from hair cells into the gelatinous cupula. Diagram from Butler and Maruska 2015. B) Scanning electron micrograph of diamond-shaped canal neuromast MD1 in Aulonocara stuartgranti, showing location of sensory hair cells in sensory strip elongated parallel to physiological orientation of hair cells and canal axis. Scale bar $=$ $10 \mu \mathrm{m}$. Image courtesy of JF Webb. 
A

St. I

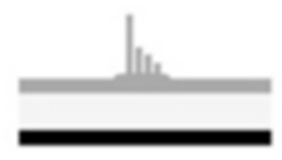

St. Ila

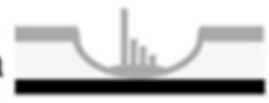

St. Ilb

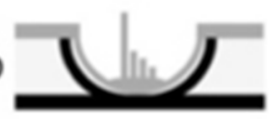

St. III

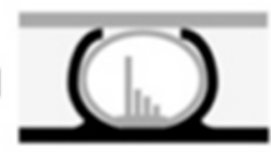

St. IV

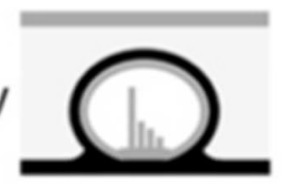

B

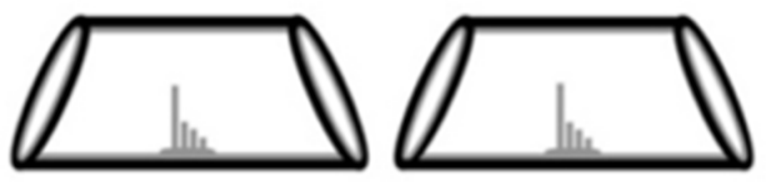

C

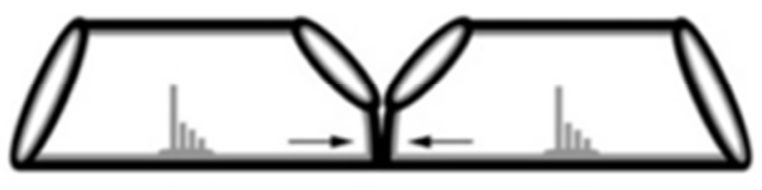

D

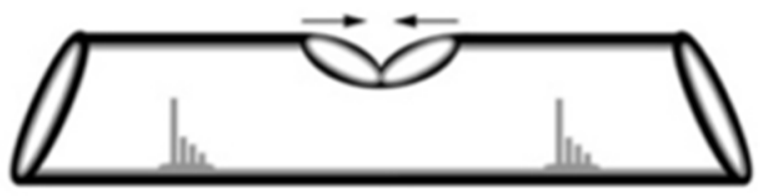

E

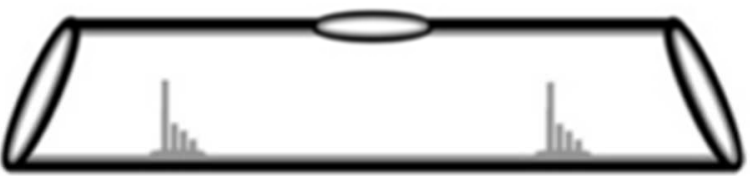

Figure 5. Neuromast-centered canal morphogenesis and fusion of adjacent canal segments. A) Stages of neuromast-centered canal morphogenesis (Stages I-IV:

Stage I - neuromast found in general epithelium, Stage IIa - neuromast sinks into depression, Stage IIb - neuromast in groove with ossified canal walls forming on either side of neuromast, Stage III - neuromast enclosed by soft tissue canal roof, Stage IV - neuromast enclosed in canal and canal roof ossified over neuromast.

Canal morphogenesis continues with the gradual fusion of adjacent canal segments. Adjacent canal segments grow toward one another (B) and make contact (C). The two adjacent segments fuse (D), leaving a pore between them (E), thus forming a continuous lateral line canal. From Bird and Webb (2014). 

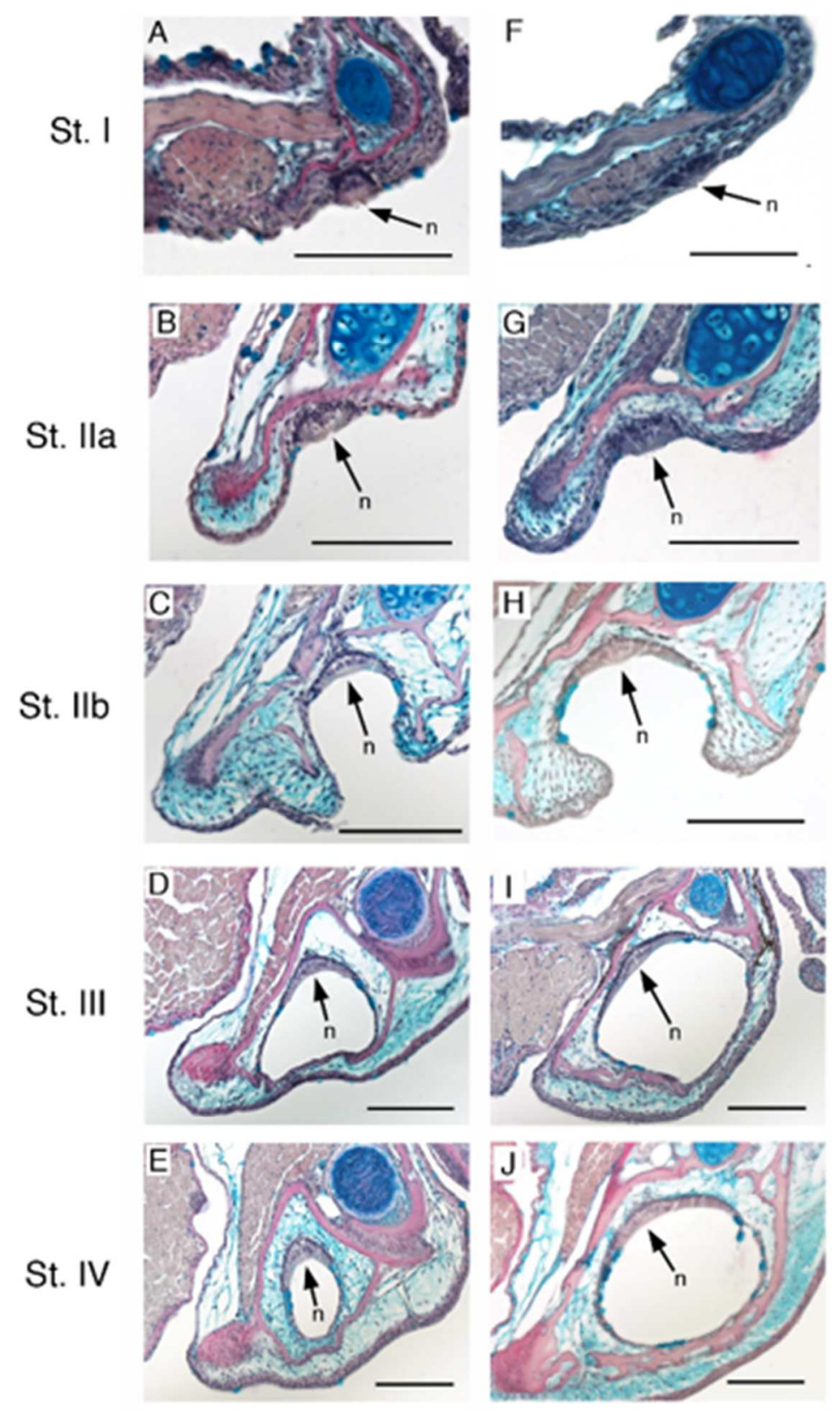

Figure 6. Stages of neuromast-centered canal morphogenesis. Development of the mandibular canal in A-E (Tramitichromis sp.), narrow lateral line canals and in F-J (Aulonocara stuartgranti) widened lateral line canals. Developmental stages are illustrated schematically in Figure 5. Pink = bone, blue $=$ Meckel's cartilage, mucous cells, or loose connective tissue. $\mathrm{N}=$ center of neuromast, indicating location of hair cells (sensory strip). All scale bars $=100 \mu \mathrm{m}$. From Bird and Webb 2014. 


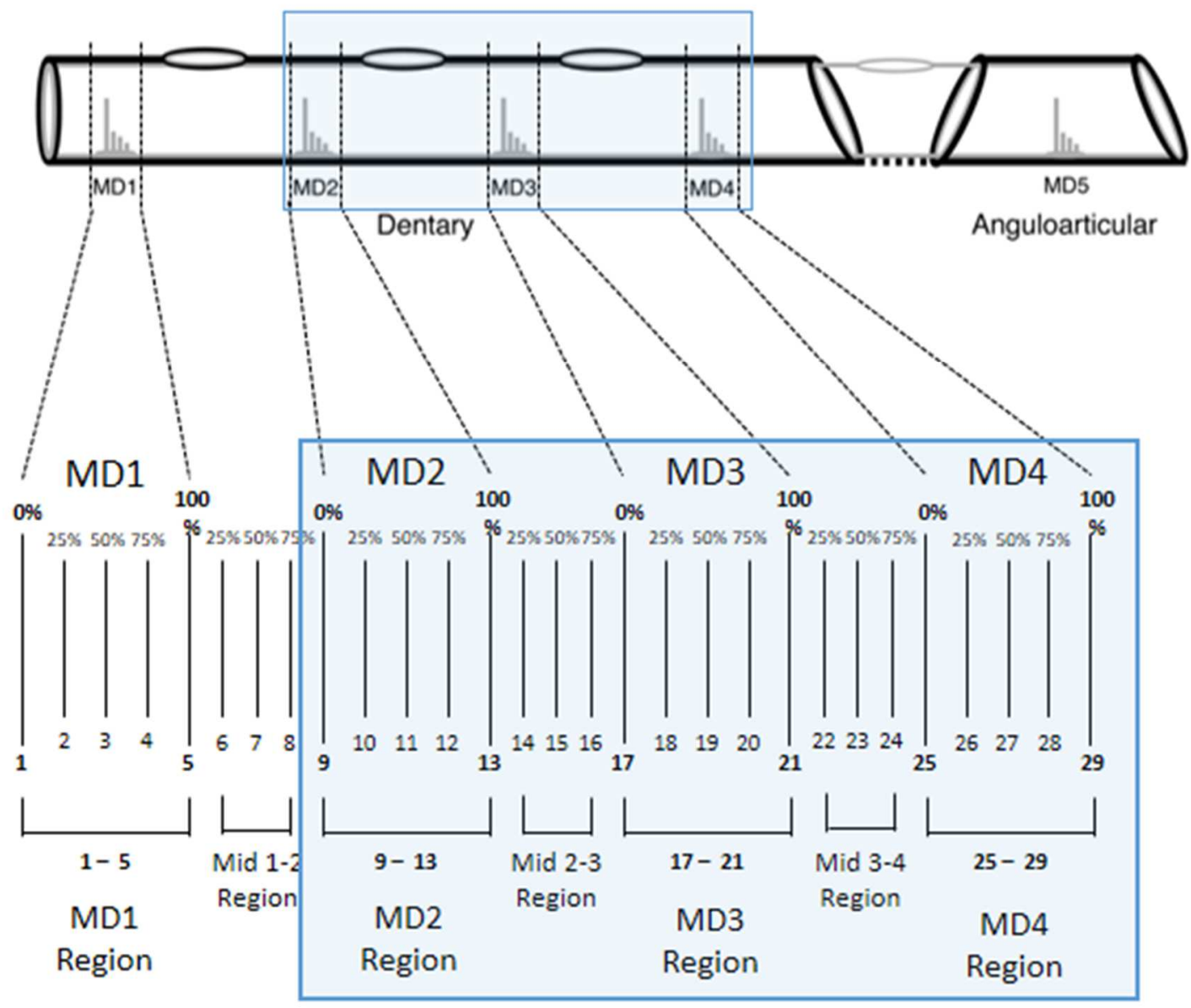

Figure 7. Distribution of canal neuromasts in the mandibular canal in relation to the positions defined for analysis. Top $=$ Schematic representation of the morphology of the mandibular lateral line canal modified from Bird and Webb (2014). Five canal neuromasts (MD1-5) are found in canal portions in the dentary (MD1-4) and the anguloarticular (MD5). Bottom = Representation of the 29 positions defined for analysis of osteoblast and osteoclast activity along the length of the mandibular canal. See text for additional details. The focal area for this study, MD2-4, is shown in the blue rectangle. 

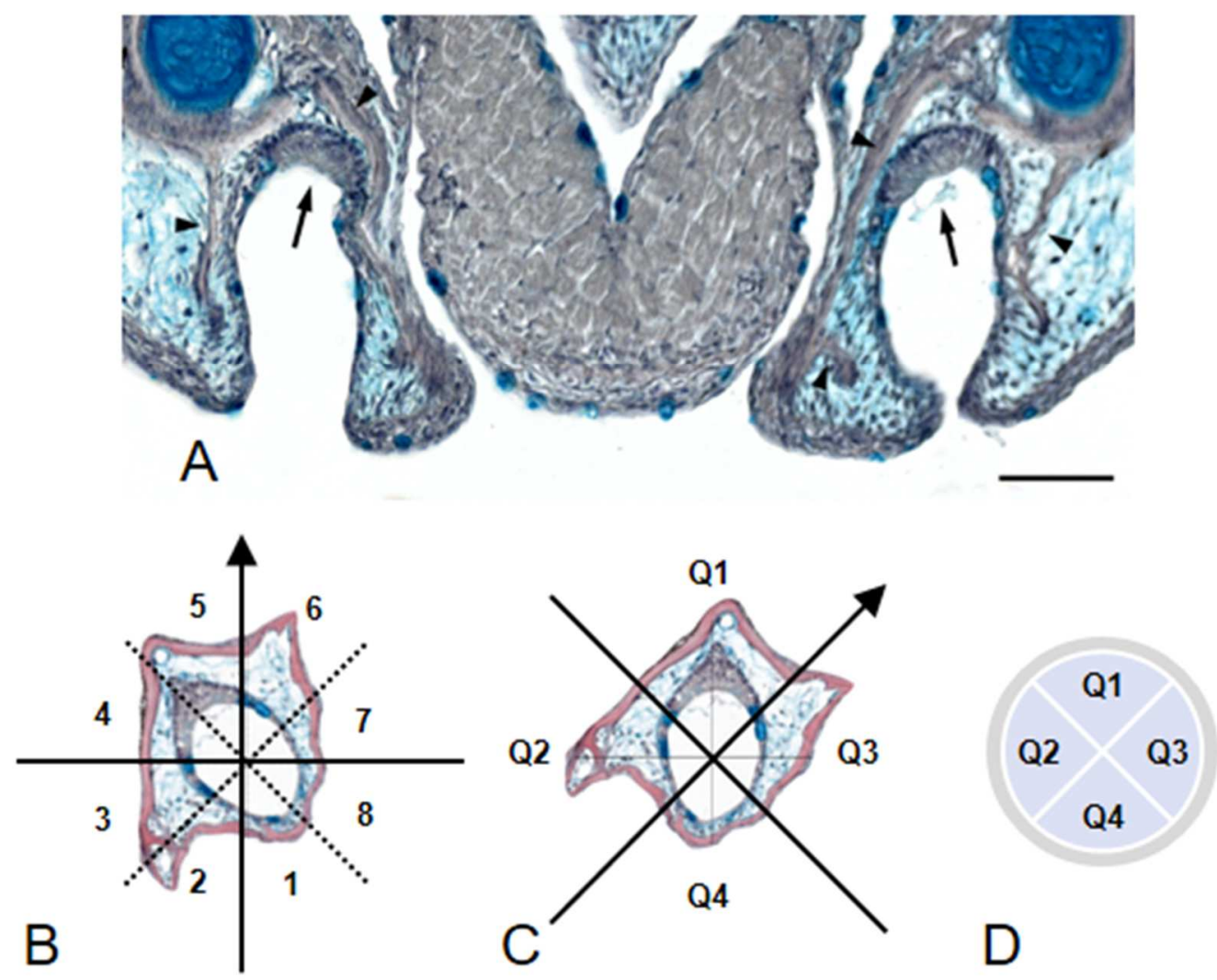

Figure 8. Method for analysis of osteoblast and osteoclast activity using AP and TRAP staining. A) Transverse section through the mandible of Aulonocara stuartgranti showing the left and right mandibular (MD) canals and MD1 neuromasts in both left and right $\mathrm{MD}$ canals. In this section, both canals are at Stage IIb. (arrows = center of neuromast, indicating location of hair cells (sensory strip), arrowheads = bone, $\mathrm{m}=$ Meckel's cartilage, blue $=$ Meckel's cartilage, mucous cells, or loose connective tissue). Scale bar $=100 \mu \mathrm{m} . \mathrm{B}$ ) Isolated right mandibular canal showing the radial grid of eight octants used to intitially collect osteoblast and osteoclast activity data around canal circumference. C) The same grid with pairs of octants combined to form the four quadrants ultimately used for analysis. Quadrant 1 represents the canal floor (under or behind the neuromast), Quadrants 2 and 3 correspond to the medial and lateral canal walls, and Quadrant 4 corresponds to the canal roof (opposite the neuromast). Note that the section has been rotated compared to (B) so that dorsal is no longer up, but offset $45^{\circ}$ clockwise. D) Example of the diagrammatic representation of a section used to analyze the radial distribution of osteoblast and osteoclast activity. If this section had been stained for either osteoblast or osteoclast activity, the amount in each quadrant would be indicated by the color of the quadrant, with darker shades representing higher activity levels. See Figures 15, 16, 18, 19, 21, 22. 

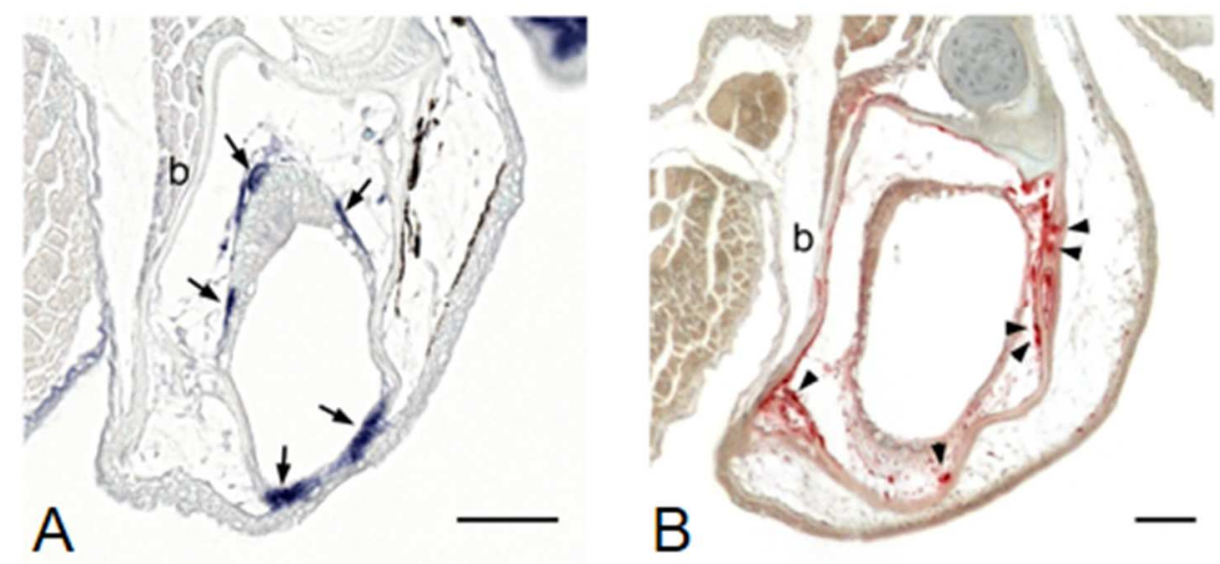

Figure 9. Osteoblast and osteoclast activity in the mandibular canal of Aulonocara stuartgranti. Transverse sections through the right mandibular canal of Aulonocara stuartgranti showing (A) AP staining for osteoblast activity and (B) TRAP staining for osteoclast activity. Note the diffuse pattern of AP staining as compared to the punctate pattern of TRAP staining of osteoclast nuclei. $\mathrm{b}=$ bone, arrows $=\mathrm{AP}$ staining (osteoblast activity), arrowheads $=$ TRAP staining (osteoclast activity), scale bars $=50 \mu \mathrm{m}$. 

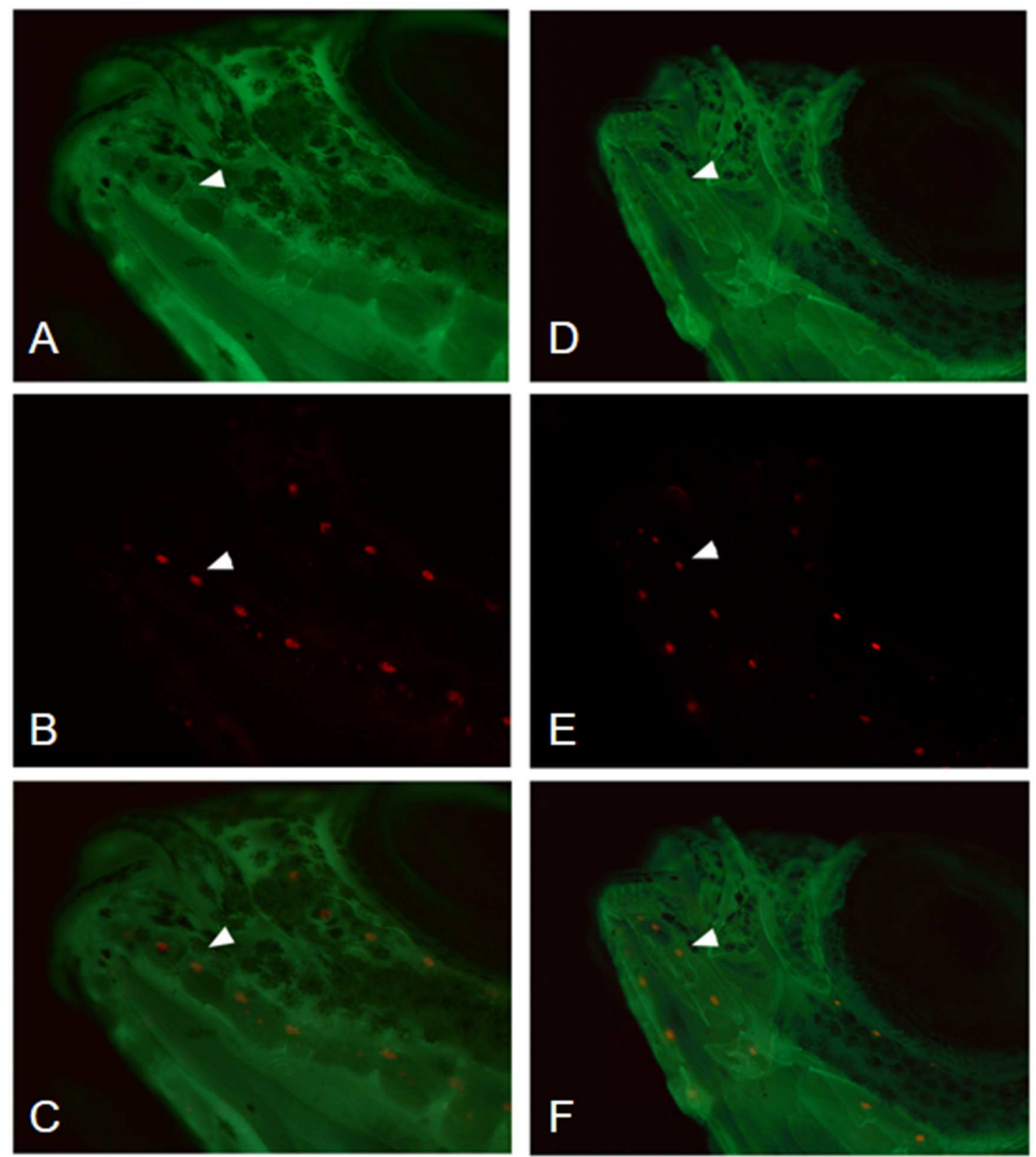

Figure 10. Vital stains used to simultaneously visualize bone and neuromasts. $16 \mathrm{~mm}$ SL Aulonocara stuartgranti (A-C) and Tramitichromis sp. (D-F) stained with calcein and 4-di-2-ASP and imaged with epifluorescence at an oblique angle to show the mandibular canal. (A, D) New bone stained green by calcein imaged using the GFP filter, and $(B, E)$ neuromasts stained orange by 4-di-2-ASP imaged using the DS-red filter. $(\mathrm{C}, \mathrm{F})$ The two images combined using Photoshop to show the distribution of neuromasts in relation to the progression of canal development. Note some segments are unenclosed (Stage I or IIb), while others are fully ossified (Stage IV) Arrowheads $=$ MD3 in each image, both Stage IV. 


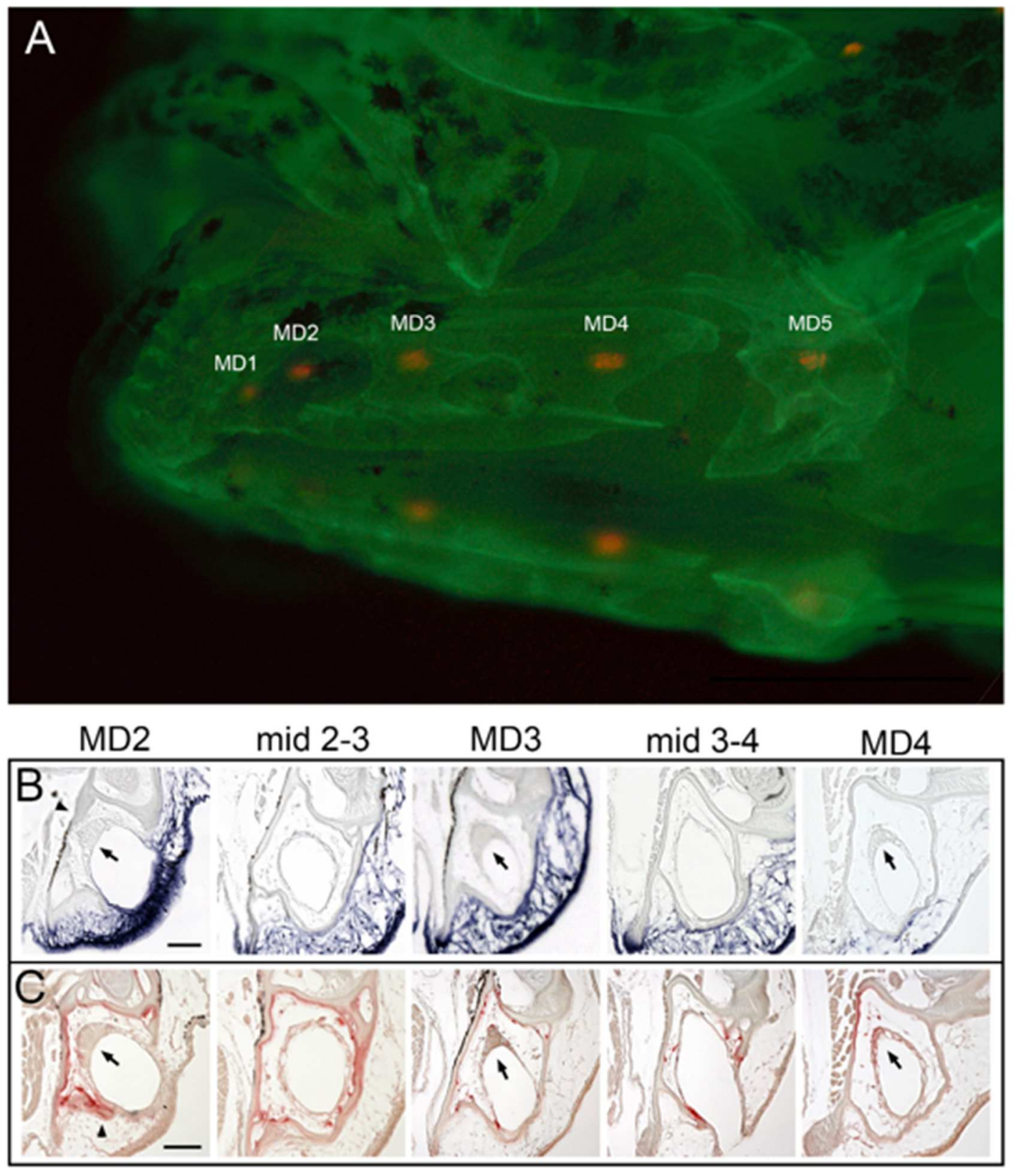

Figure 11. Vital and histochemical staining methods in $16 \mathrm{~mm}$ SL Tramitichromis sp. A) Vital staining with calcein and 4-di-2-ASP shows mandibular neuromasts (MD1-5) within the developing mandibular canal, in lateroventral view. Scale bar $=$ $1 \mathrm{~mm}$. Sections from $16 \mathrm{~mm}$ SL fish along the canal length showing radial distribution of (B) osteoblast and (C) osteoclast activity around the canal circumference (see also Figure 9). Arrows = neuromasts, arrowheads $=$ bone, scale bars $=50 \mu \mathrm{m}$. 

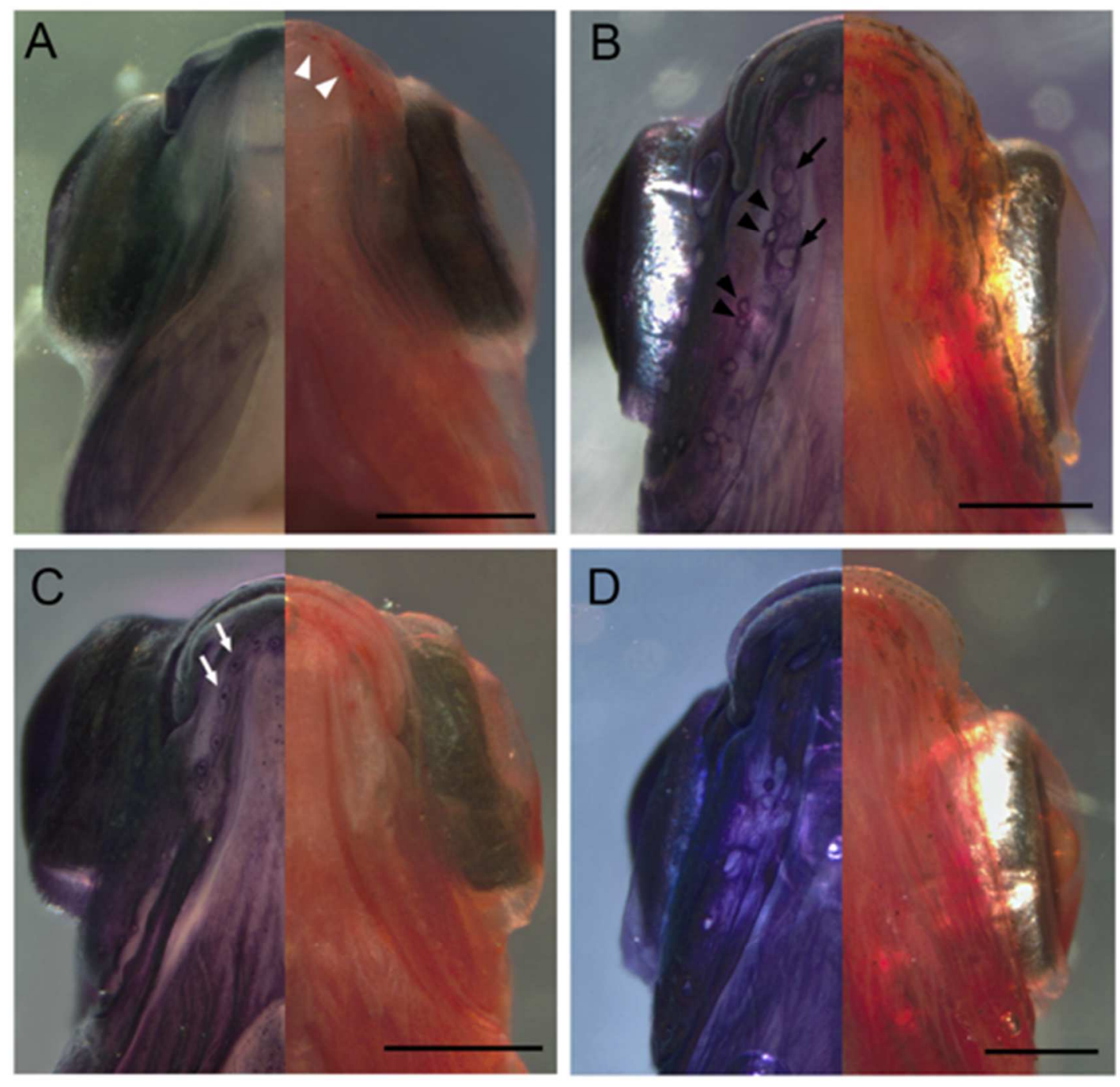

Figure 12. Whole mount staining for osteoblast and osteoclast activity.

Aulonocara stuartgranti and Tramitichromis sp. stained with AP (blue) or TRAP (red) to visualize osteoblast and osteoclast activity on left and right in each split image, respectively. A) $8 \mathrm{~mm}$ SL Aulonocara, B) $8 \mathrm{~mm}$ SL Tramitichromis, C) 12 $\mathrm{mm}$ SL Aulonocara, D) $12 \mathrm{~mm}$ SL Tramitichromis. White arrowheads $=$ TRAP staining near $\mathrm{MD}$ neuromasts, black arrows = pores of $\mathrm{MD}$ canal, black arrowheads $=$ ring of AP staining around superficial neuromasts (see also Figure 13), white arrows $=M D$ neuromasts. $A, B, E, F$ : Scale bars $=0.5 \mathrm{~mm} . \mathrm{C}, \mathrm{D}, \mathrm{G}, \mathrm{H}$ : Scale bars $=1 \mathrm{~mm}$. 

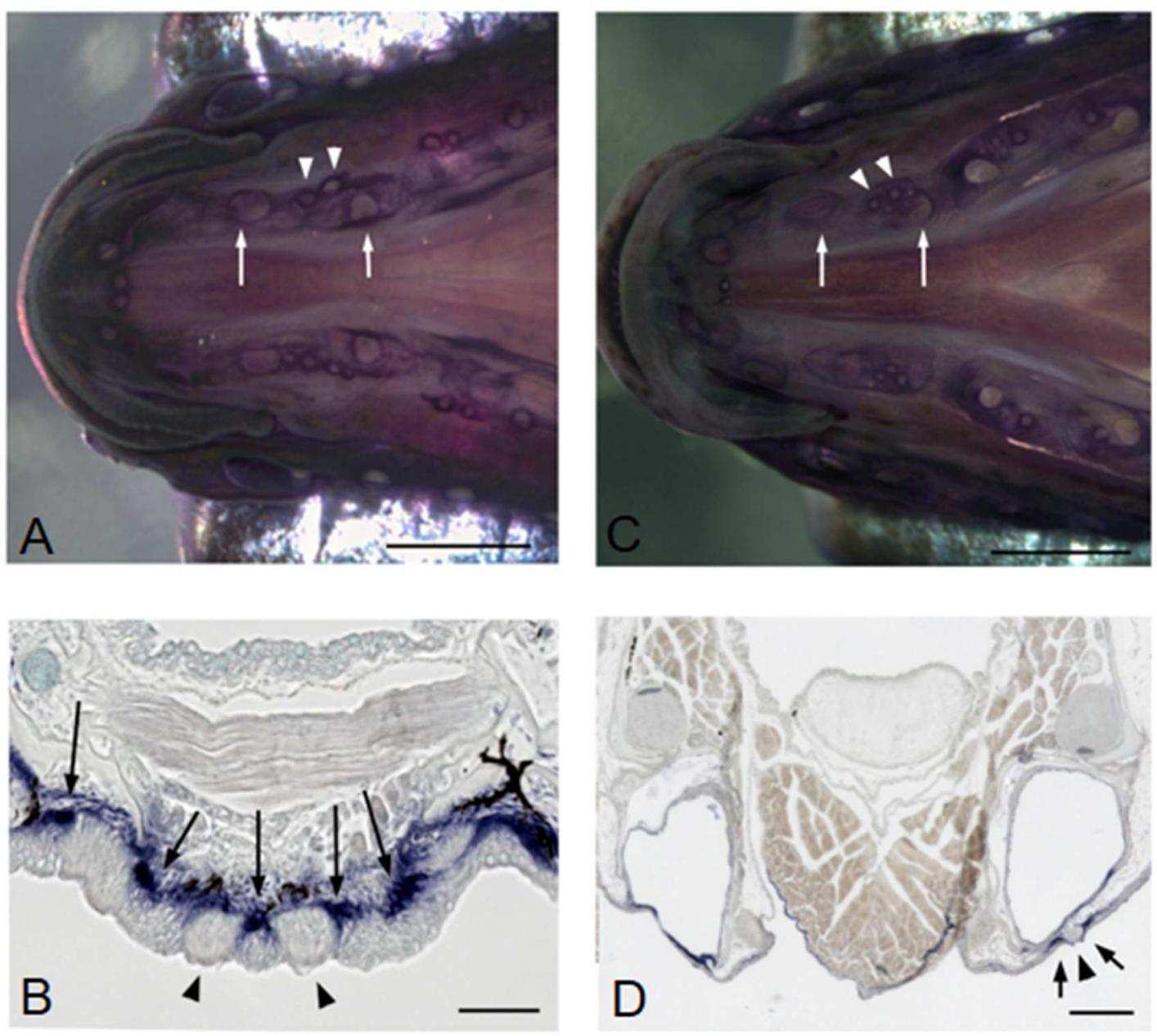

Figure 13. Ring of AP staining around superficial and canal neuromasts. A ring of AP staining was observed around neuromasts in small Aulonocara stuartgranti and Tramitichromis sp. In $12 \mathrm{~mm} \mathrm{SL}$ Aulonocara this staining pattern is evident in (A) the ventral view of the whole mount stained specimen. B) In a transverse section through the $\mathrm{MD} 1$ region, the spots of $\mathrm{AP}$ staining on either side of both superficial and canal neuromasts indicate a ring pattern of staining observed in whole fish (see Figure 12). In larger animals, $20 \mathrm{~mm} \mathrm{SL}$ Aulonocara, (C) the whole mount ventral view shows rings of AP staining around the superficial neuromasts, but the canal neuromasts are not visible (inside the canal). D) In histological section, both canals show a canal neuromast with no ring staining pattern, and a superficial neuromast on the roof of the right canal, showing a ring of AP staining. White arrows $=\mathrm{MD}$ canal pores, white arrowheads $=$ ring of $\mathrm{AP}$ staining around superficial neuromasts, black arrowheads = superficial neuromasts in section, black arrows $=$ ring of AP staining in section. $\mathrm{A}, \mathrm{C}$; scale bars $=0.5 \mathrm{~mm}$. B, D: Scale bars $=50 \mu \mathrm{m}$. 


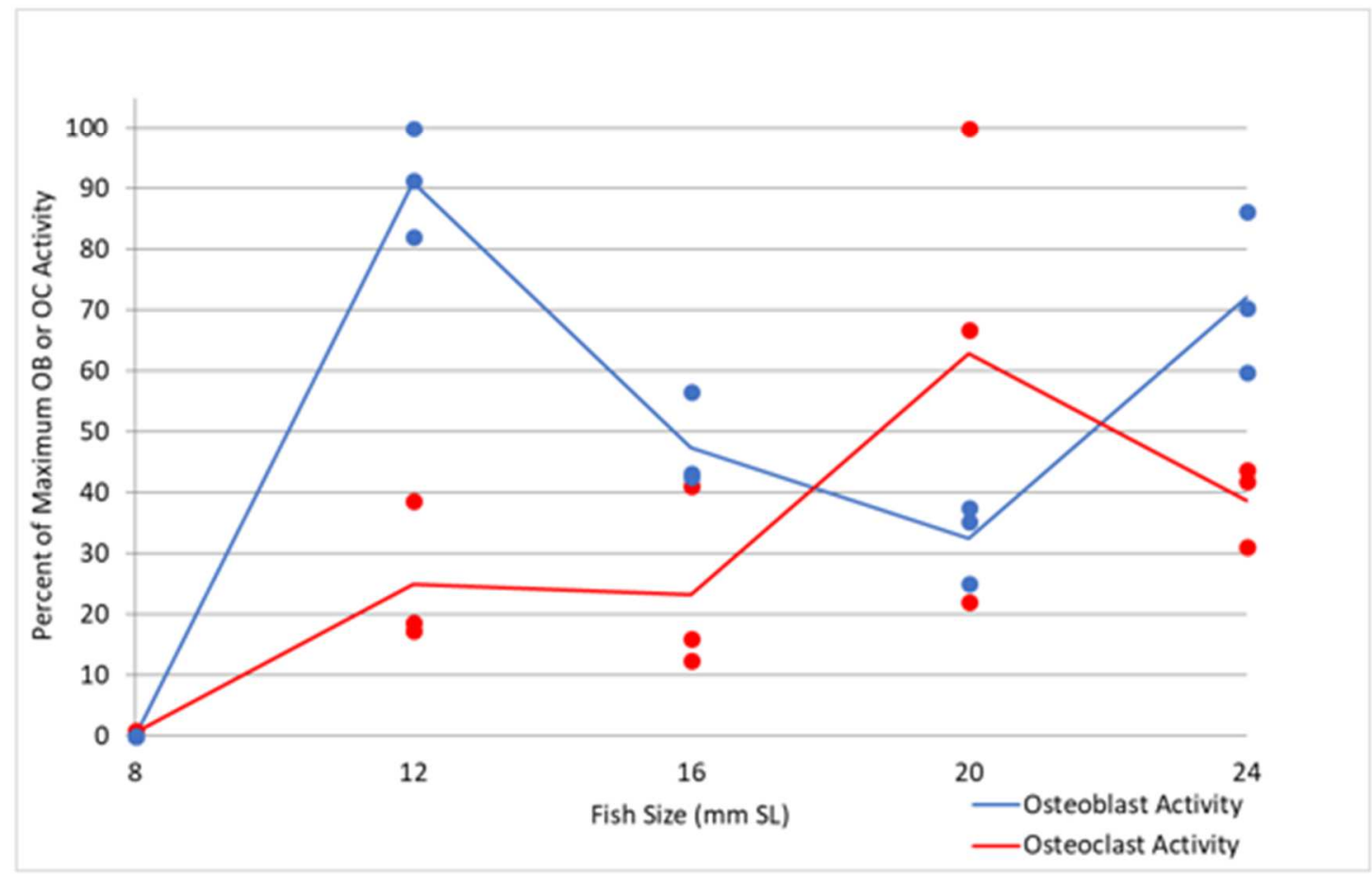

Figure 14. Osteoblast and osteoclast activity through ontogeny in Aulonocara stuartgranti. One pulse of osteoblast activity (bone deposition) at $12 \mathrm{~mm} \mathrm{SL}$ and a pulse of osteoclast activity (bone resorption) at $20 \mathrm{~mm} \mathrm{SL}$ accompanied by bone deposition. Each data point represents the percentage of the maximum total osteoblast or osteoclast activity in all four quadrants at all 29 positions for each individual fish $(n=3)$. The solid line represents the trend in the mean for all three fish at each fish size $(8,12,16,20,24 \mathrm{~mm} \mathrm{SL})$. 


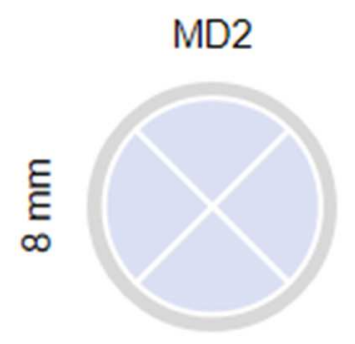

$$
\text { Mid 2-3 }
$$

MD3

Mid 3-4

MD4
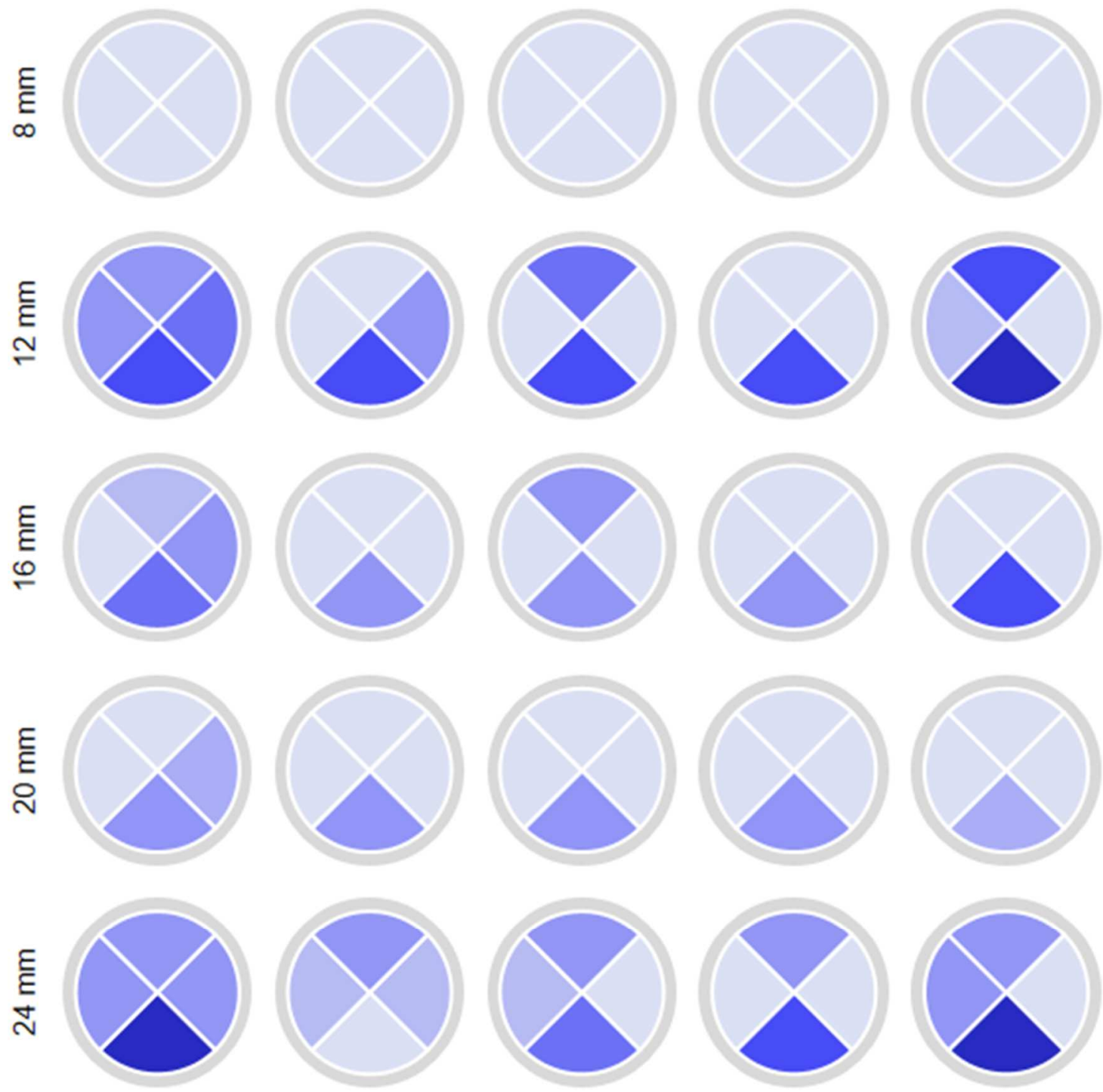

\begin{tabular}{|c|c|}
\hline Color & Score \\
\hline & 0 \\
\hline & 1 \\
\hline & 2 \\
\hline & 3 \\
\hline & 4 \\
\hline
\end{tabular}

Figure 15. Osteoblast activity through ontogeny along canal length (MD2-MD4) in Aulonocara stuartgranti. Diagrammatic representations of the median level of osteoblast activity in the four quadrants (see Figure 8) in transverse sections using a colorimetric scale ( $\mathrm{n}=3$ fish). See text for additional explanation. 


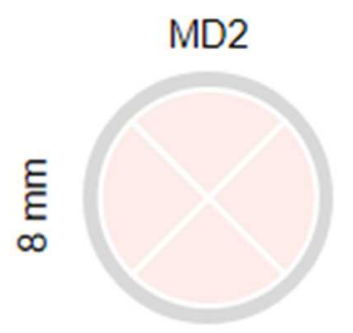

$$
\text { Mid 2-3 }
$$

MD3

Mid 3-4

MD4
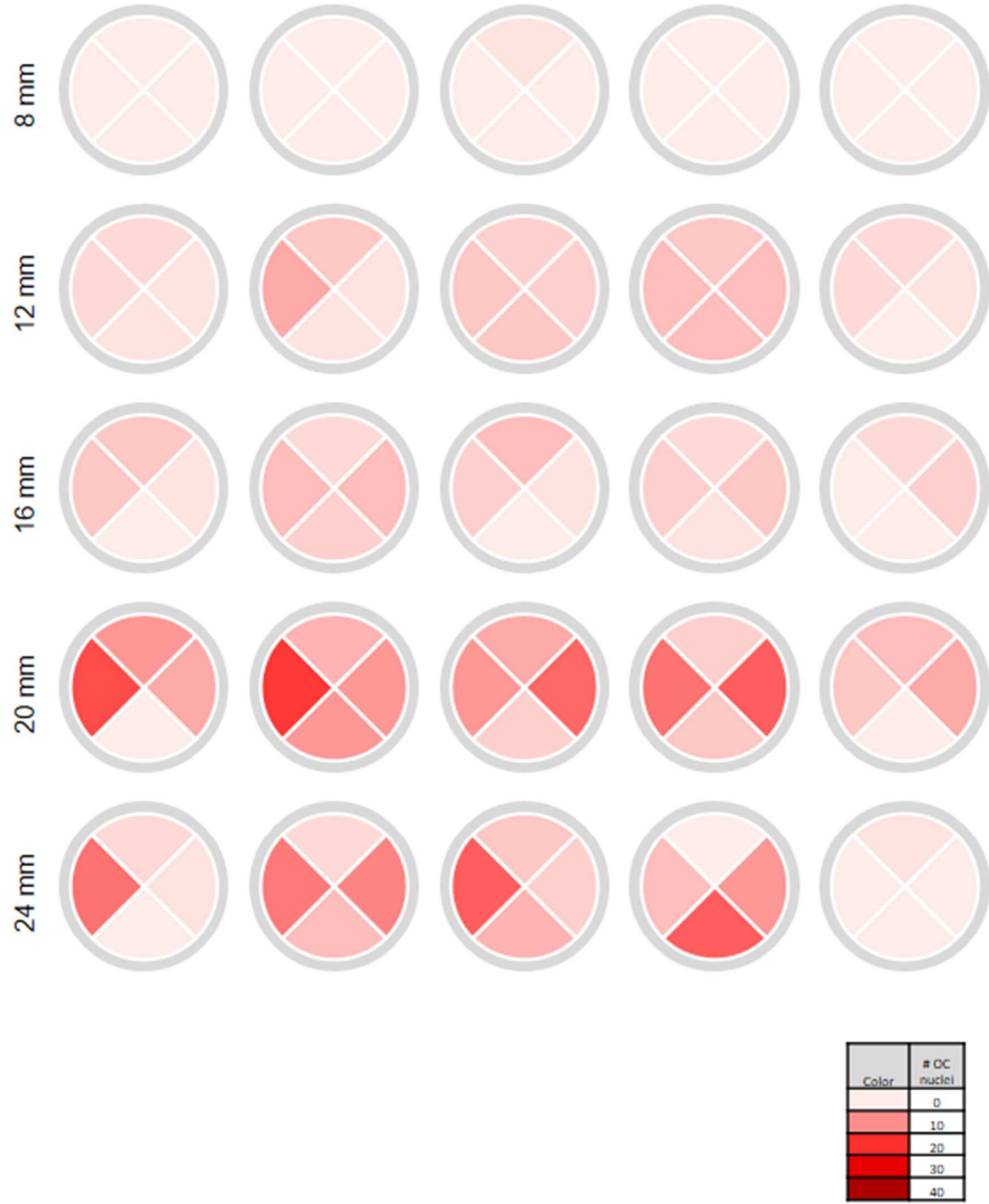

Figure 16. Osteoclast activity through ontogeny along canal length in Aulonocara stuartgranti. Diagrammatic representations of the median level of osteoclast activity in the four quadrants (see Figure 8) in transverse sections using a colorimetric scale ( $\mathrm{n}=3$ fish). See text for additional explanation. 


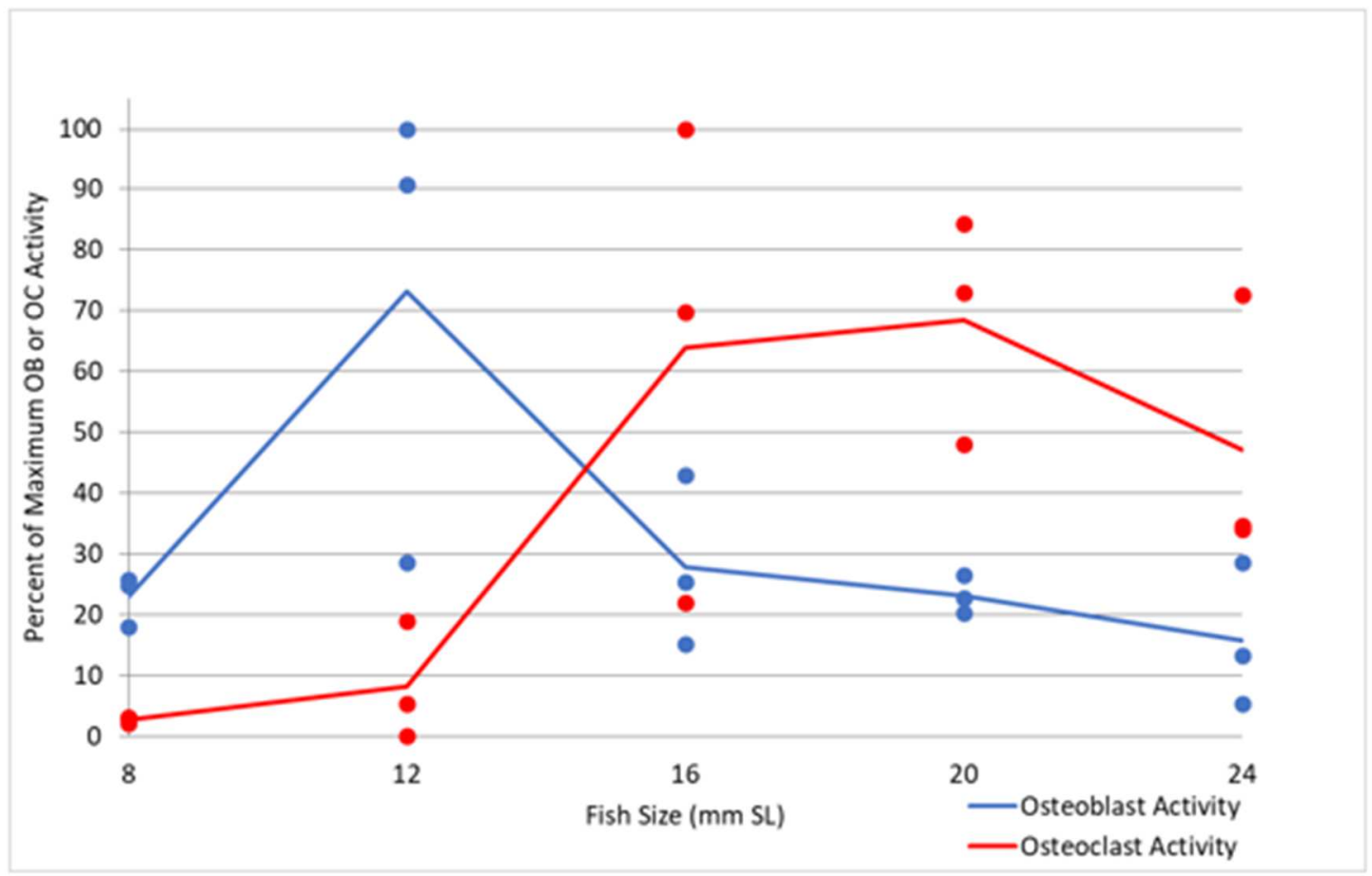

Figure 17. Osteoblast and osteoclast activity through ontogeny in Tramitichromis sp. One pulse of osteoblast activity (bone deposition) at $12 \mathrm{~mm} \mathrm{SL}$ and a pulse of osteoclast activity (bone resorption) at $16-20 \mathrm{~mm} \mathrm{SL}$. . Each data point represents the percentage of the maximum total osteoblast or osteoclast activity in all four quadrants at all 29 positions for each individual fish $(n=3)$. The solid line represents the trend in the mean for all three fish at each fish size $(8,12,16,20$, $24 \mathrm{~mm} \mathrm{SL})$. 


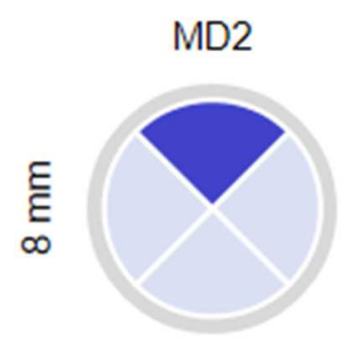

$$
\text { Mid 2-3 }
$$

MD3
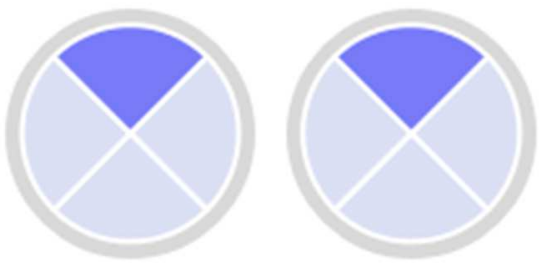

Mid 3-4

MD4
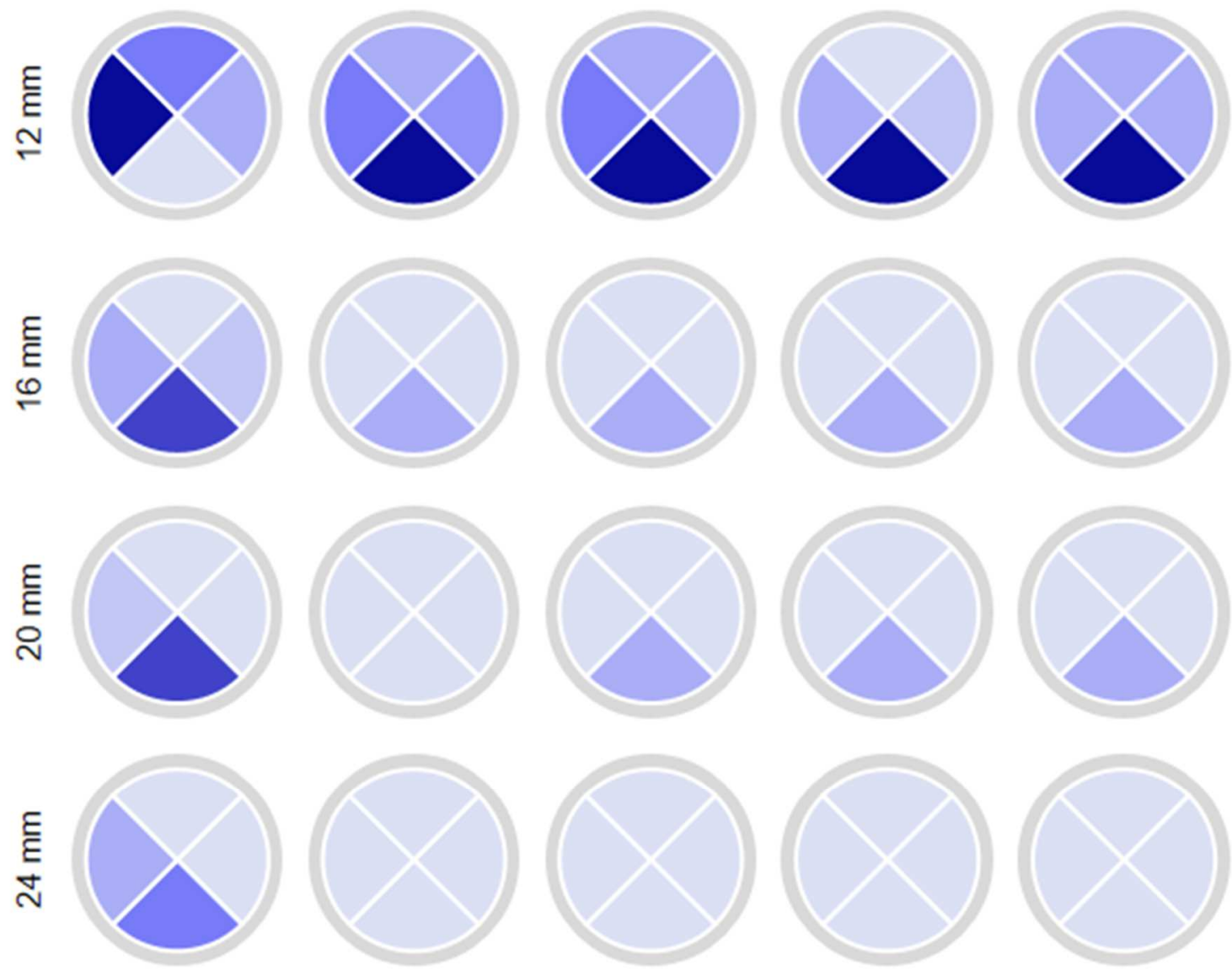

\begin{tabular}{|c|c|}
\hline Color & Score \\
\hline & 0 \\
\hline & 1 \\
\hline & 2 \\
\hline & 3 \\
\hline & 4 \\
\hline
\end{tabular}

Figure 18. Osteoblast activity through ontogeny along canal length in Tramitichromis sp. Diagrammatic representations of the median level of osteoblast activity in the four quadrants (see Figure 8) in transverse sections using a colorimetric scale ( $\mathrm{n}=3$ fish). See text for additional explanation. 


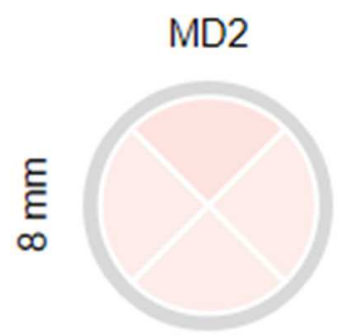

Mid 2-3

MD3

Mid 3-4

MD4
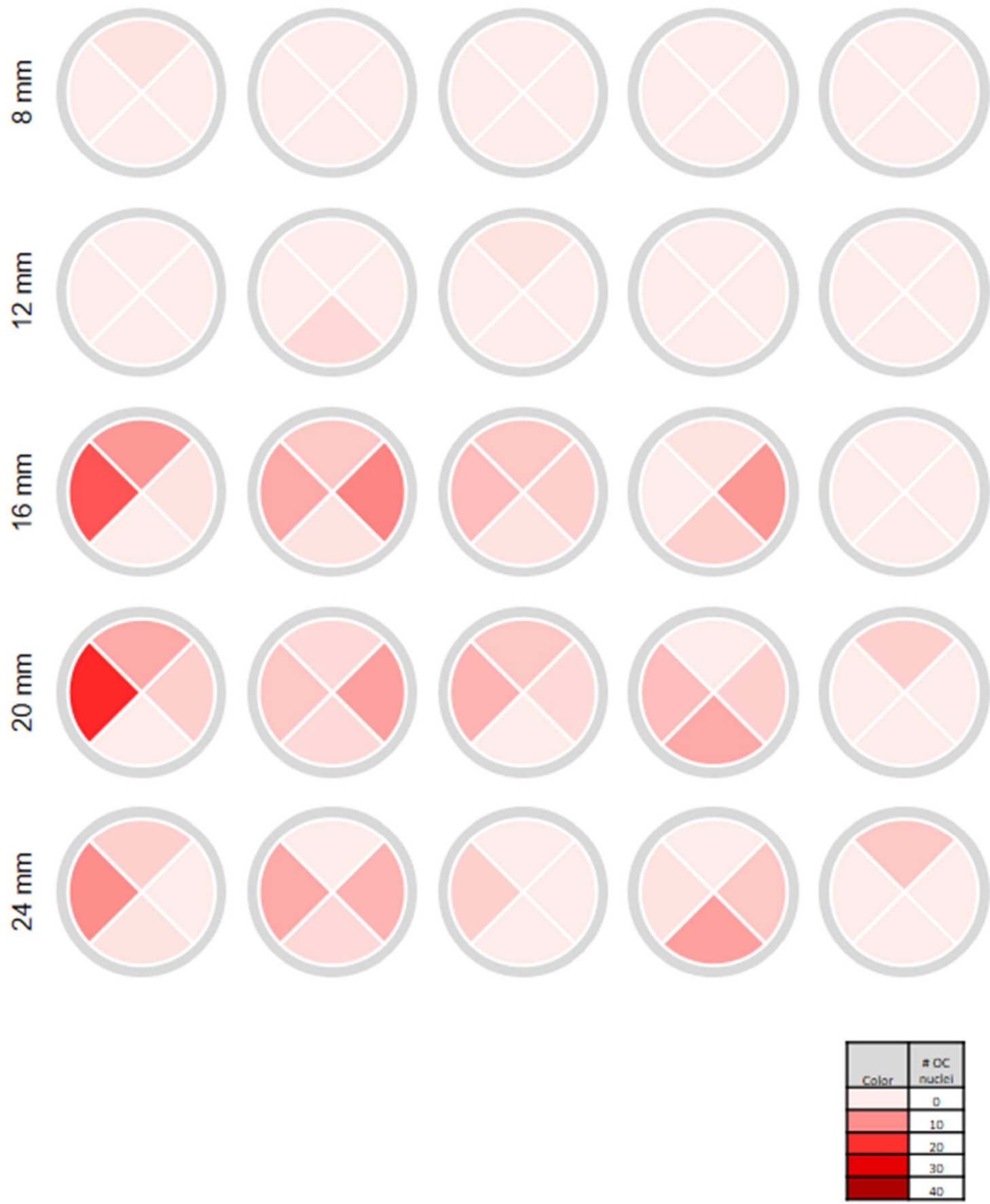

Figure 19. Osteoclast activity through ontogeny along canal length in Tramitichromis sp. Diagrammatic representations of the median level of osteoclast activity in the four quadrants (see Figure 8) in transverse sections using a colorimetric scale ( $\mathrm{n}=3$ fish). See text for additional explanation. 

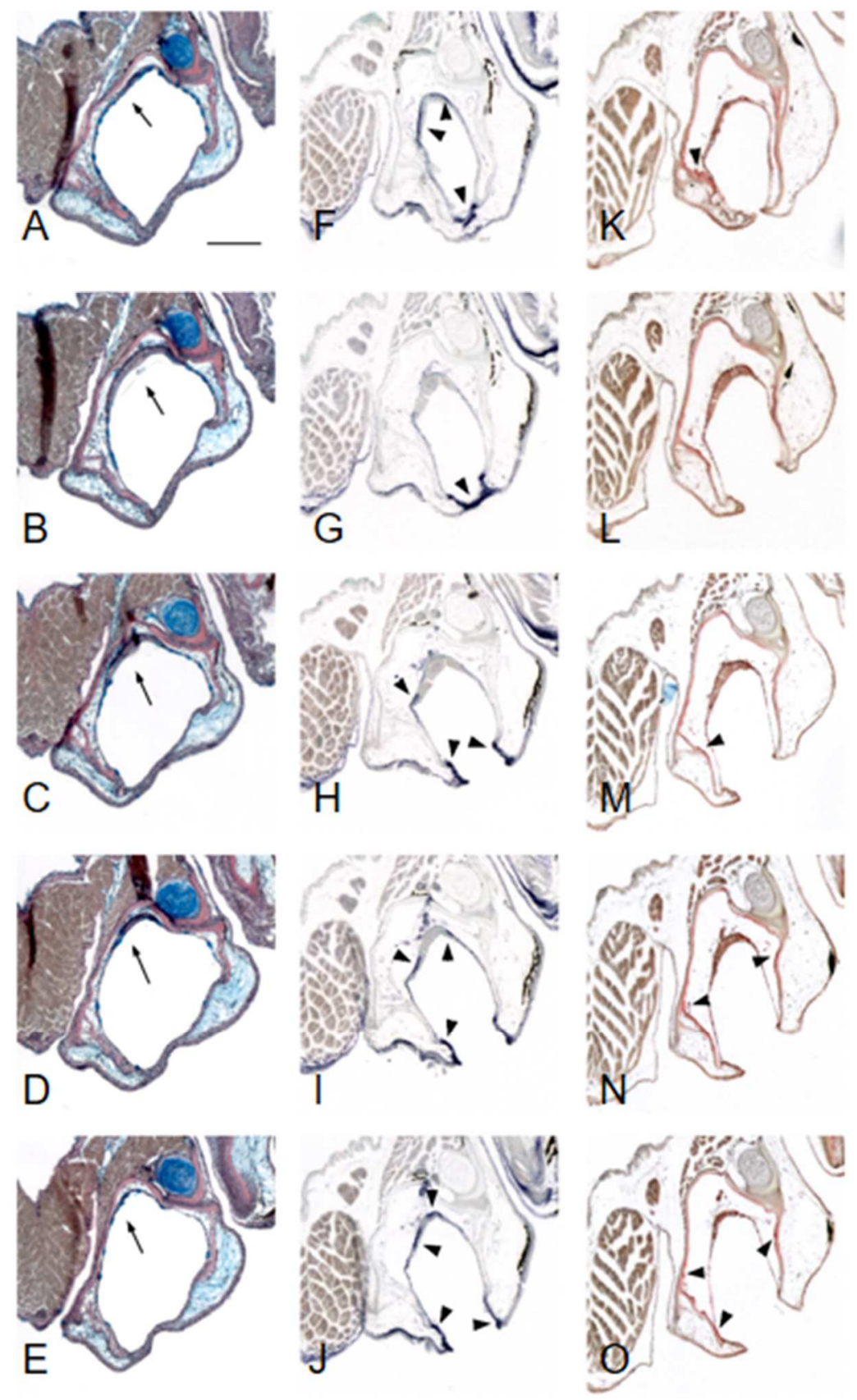

Figure 20. Osteoblast and osteoclast activity in the $\mathrm{MD} 3$ region in $16 \mathrm{~mm} \mathrm{SL}$ Aulonocara stuartgranti. Transverse histological sections through MD3 with more rostral sections to the top and caudal to the bottom (positions 17-21; the rostral end, $25 \%$ of the way through, the midpoint, $75 \%$ of the way through, and the caudal end). A-E) Sections stained with Hall's HBQ stain to show the general anatomy of the area, arrows $=$ neuromast center; (F-J) AP staining shows osteoblast activity, arrowheads = AP staining; and (K-O) TRAP staining shows osteoclast activity, arrowheads = TRAP staining. Scale bar in $A=100 \mu \mathrm{m}$, and applies to all images. 

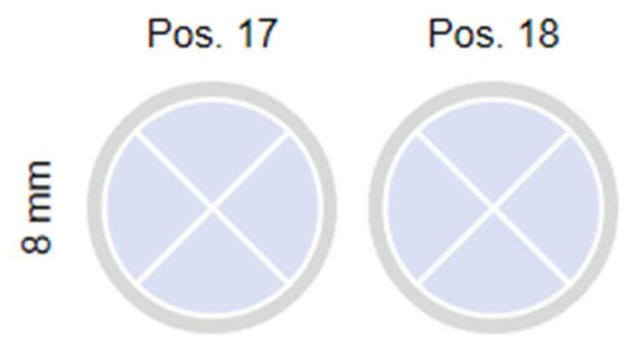

Pos. 19

Pos. 20

Pos. 21
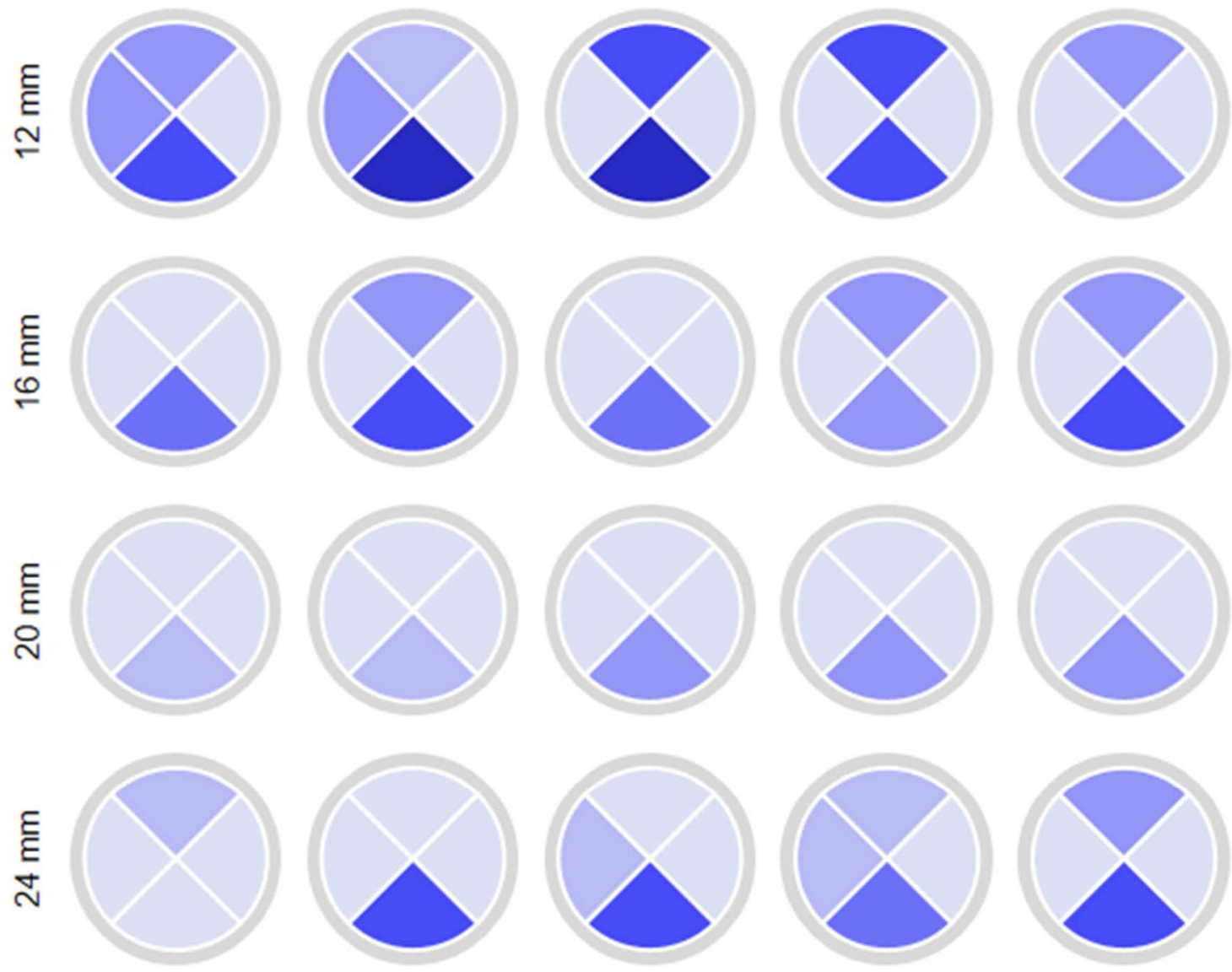

\begin{tabular}{|c|c|}
\hline Color & Score \\
\hline & 0 \\
\hline & 1 \\
\hline & 2 \\
\hline & 3 \\
\hline & 4 \\
\hline
\end{tabular}

Figure 21. Osteoblast activity in MD3 (Positions 17-21) through ontogeny in Aulonocara stuartgranti. Diagrammatic representations of the median level of osteoblast activity in four quadrants (see Figure 8 ) in transverse sections using a colorimetric scale ( $\mathrm{n}=3 \mathrm{fish}$ ). See text for additional explanation. 

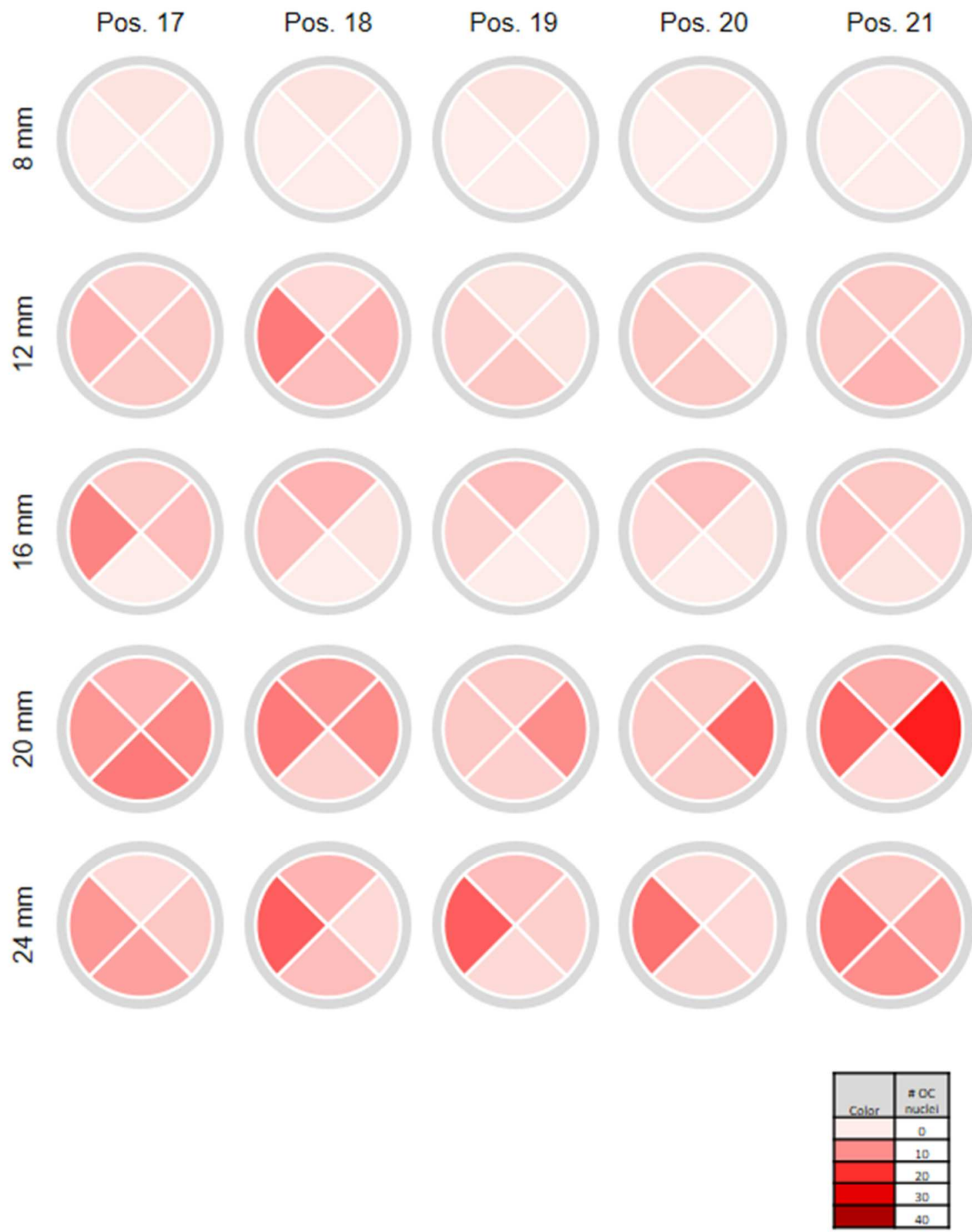

Figure 22. Osteoclast activity in MD3 (Positions 17-21) through ontogeny in Aulonocara stuartgranti. Diagrammatic representations of the median level of osteoclast activity in the four quadrants (see Figure 8) in transverse sections using a colorimetric scale ( $n=3$ fish). See text for additional explanation. 


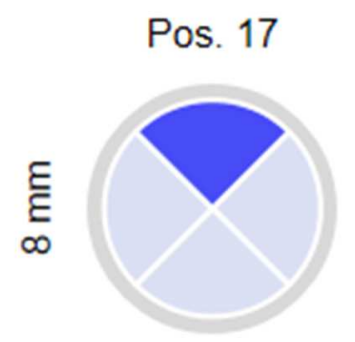

Pos. 18

Pos. 19

Pos. 20

Pos. 21
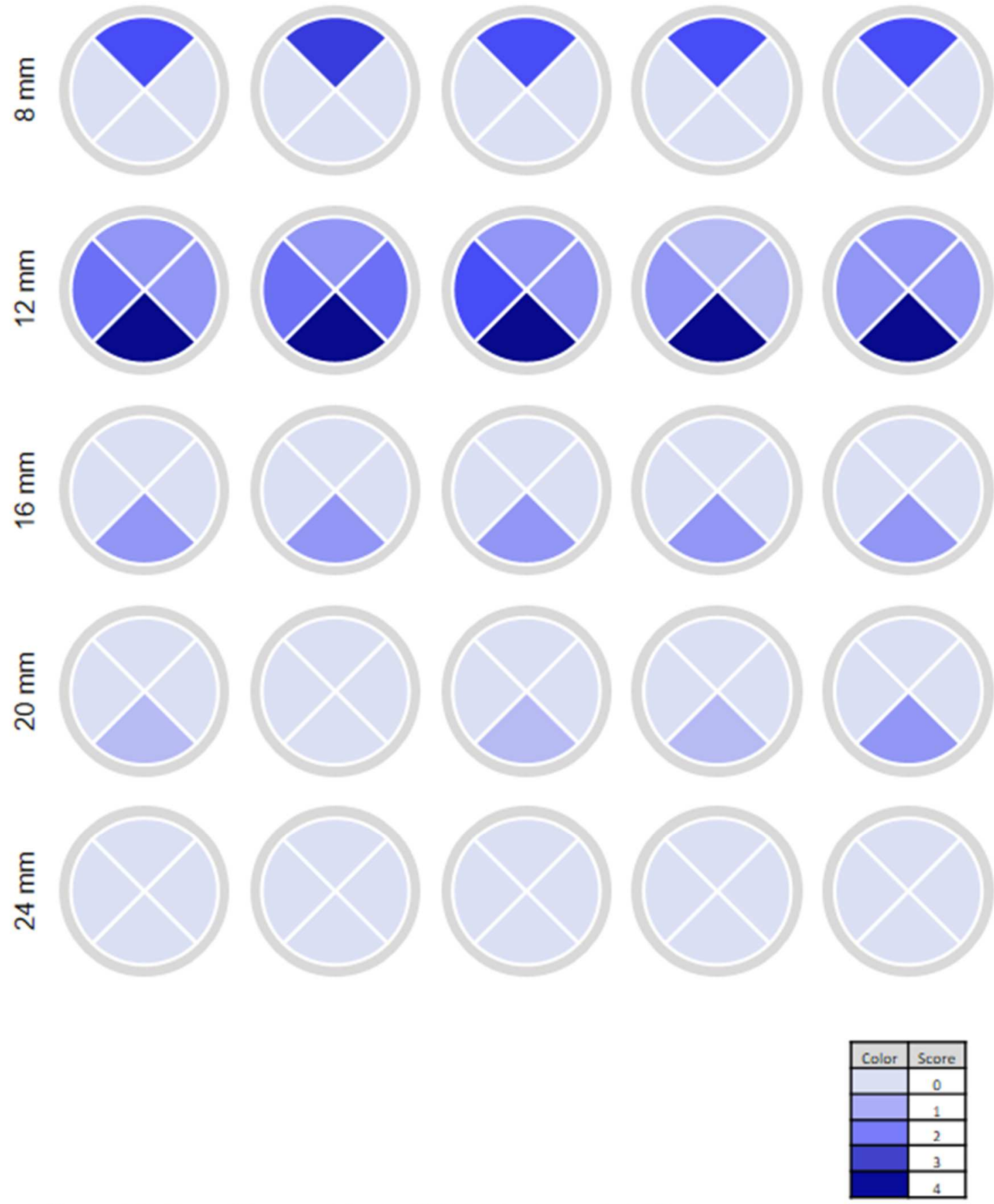

Figure 23. Osteoblast activity in MD3 (Positions 17-21) through ontogeny in Tramitichromis sp. Diagrammatic representations of the median level of osteoblast activity in the four quadrants (see Figure 8) in transverse sections using a colorimetric scale ( $\mathrm{n}=3$ fish). See text for additional explanation. 

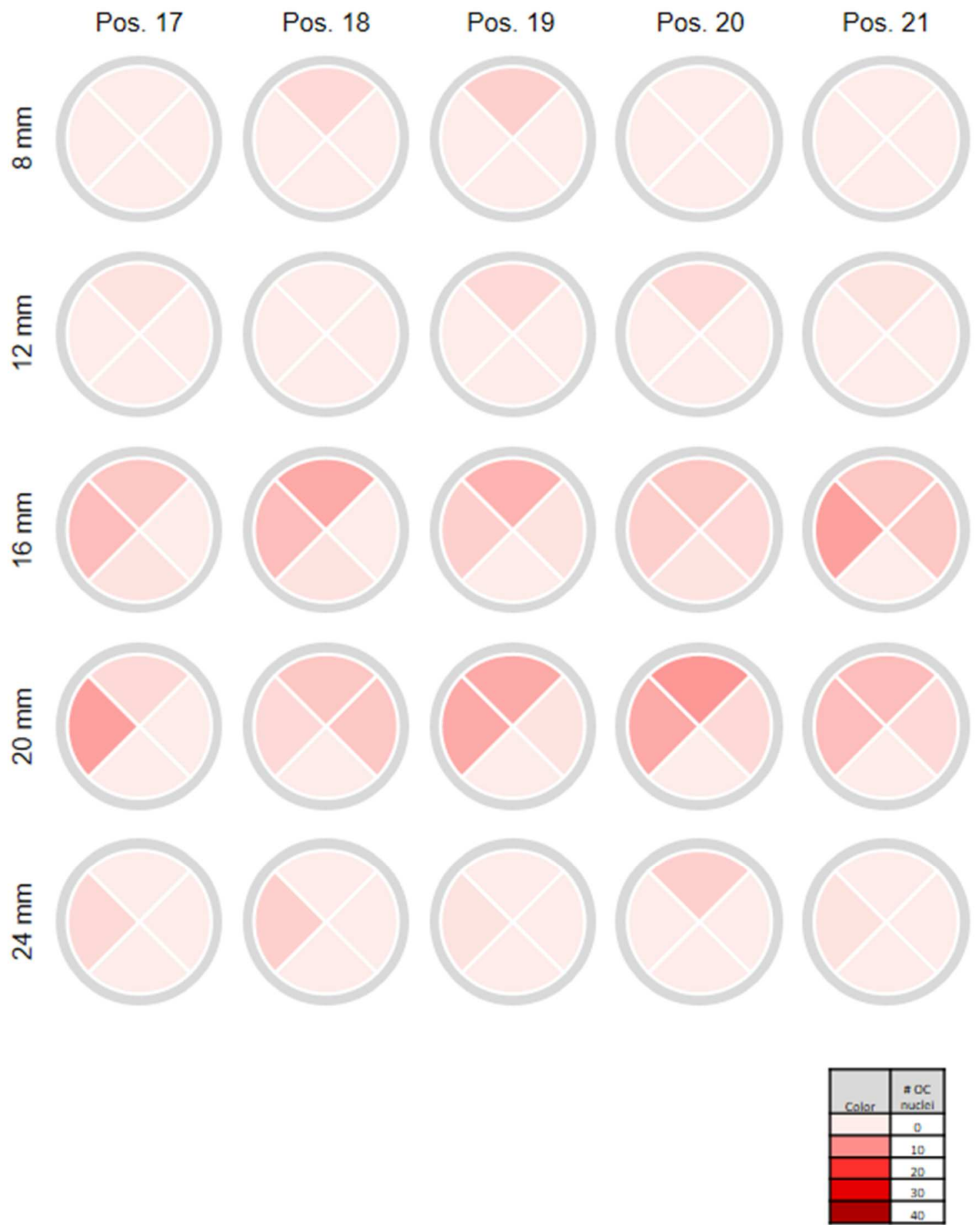

Figure 24. Osteoclast activity in MD3 (Positions 17-21) through ontogeny in Tramitichromis sp. Diagrammatic representations of the median level of osteoclast activity in the four quadrants (see Figure 8) in transverse sections using a colorimetric scale ( $\mathrm{n}=3$ fish). See text for additional explanation. 


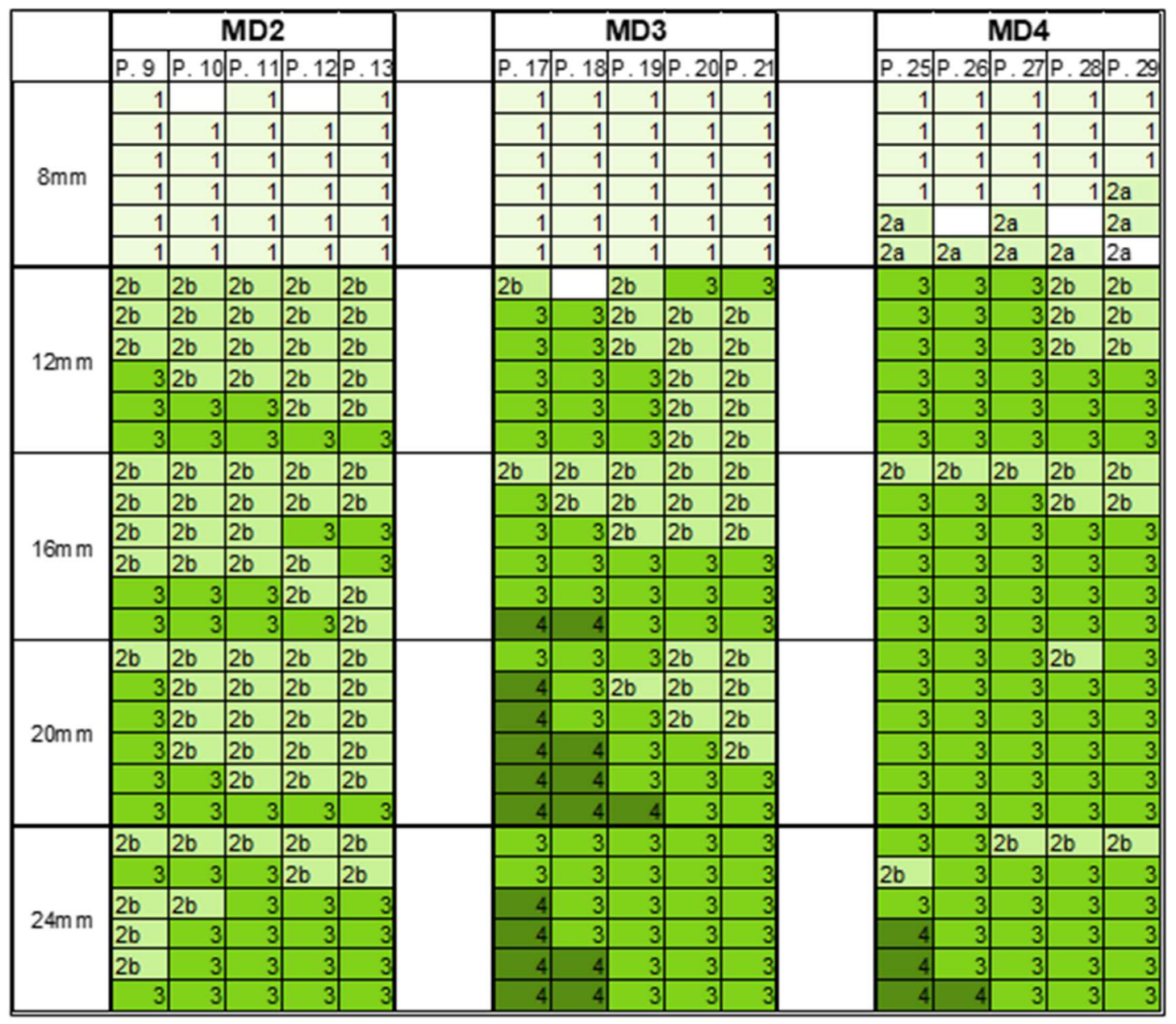

Figure 25. Canal development in neuromast regions MD2, MD3, and MD4 in Aulonocara stuartgranti. Three AP-stained and three TRAP-stained Aulonocara were pooled to yield six replicates for each of the five size classes (total $n=30$ ). Canal enclosure stage (Tarby and Webb 2003, Webb and Shirey 2003, Bird and Webb 2014) was recorded and a colorimetric scale was applied, with darker greens representing more advanced stages of canal development. 


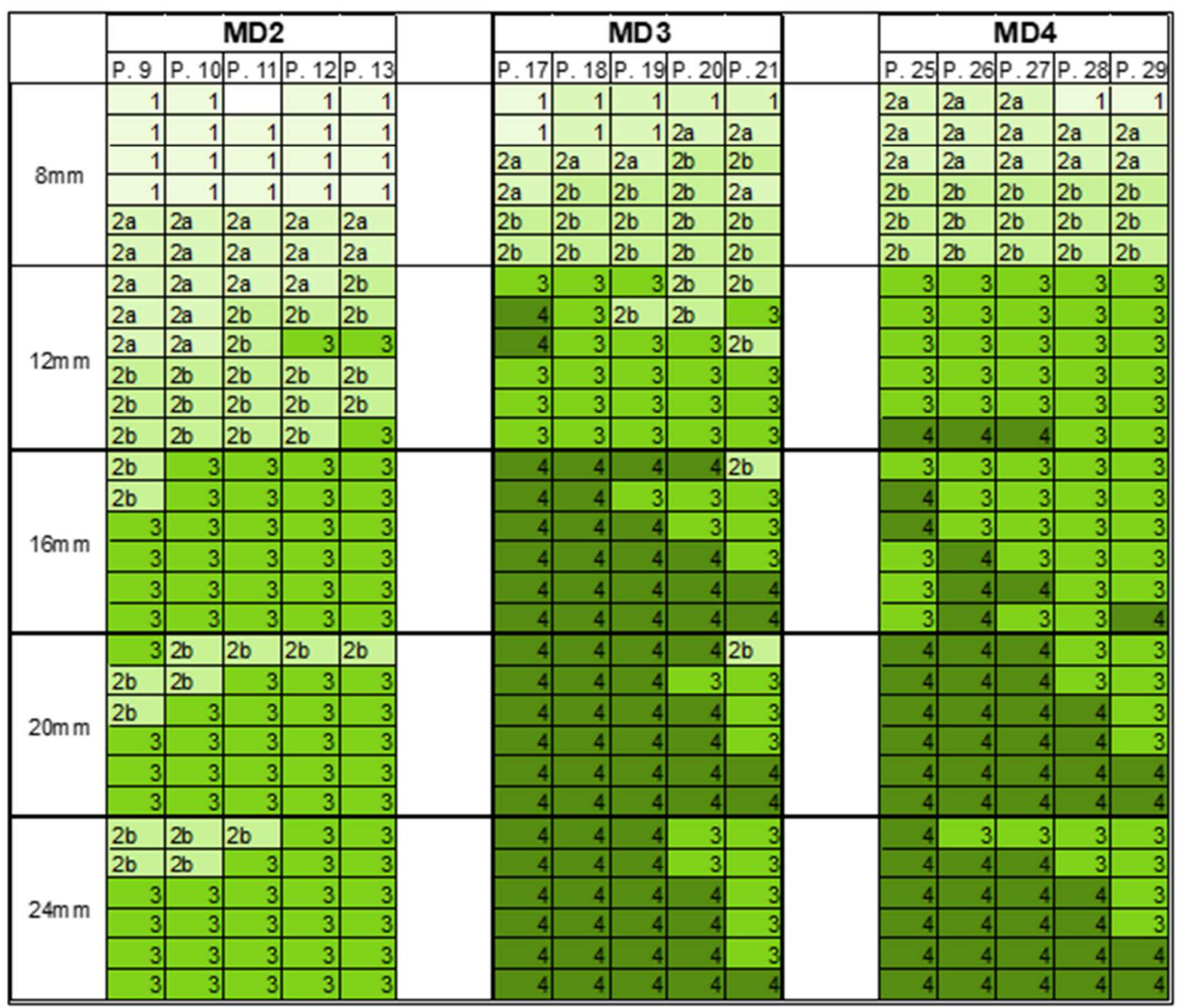

Figure 26. Canal development in neuromast regions $\mathrm{MD} 2, \mathrm{MD} 3$, and MD4 in Tramitichromis sp. Three AP-stained and three TRAP-stained Tramitichromis were pooled to yield six replicates for each of the five size classes (total $n=30$ ). Canal enclosure stage (Tarby and Webb 2003, Webb and Shirey 2003, Bird and Webb 2014) was recorded and a colorimetric scale was applied, with darker greens representing more advanced stages of canal development. 

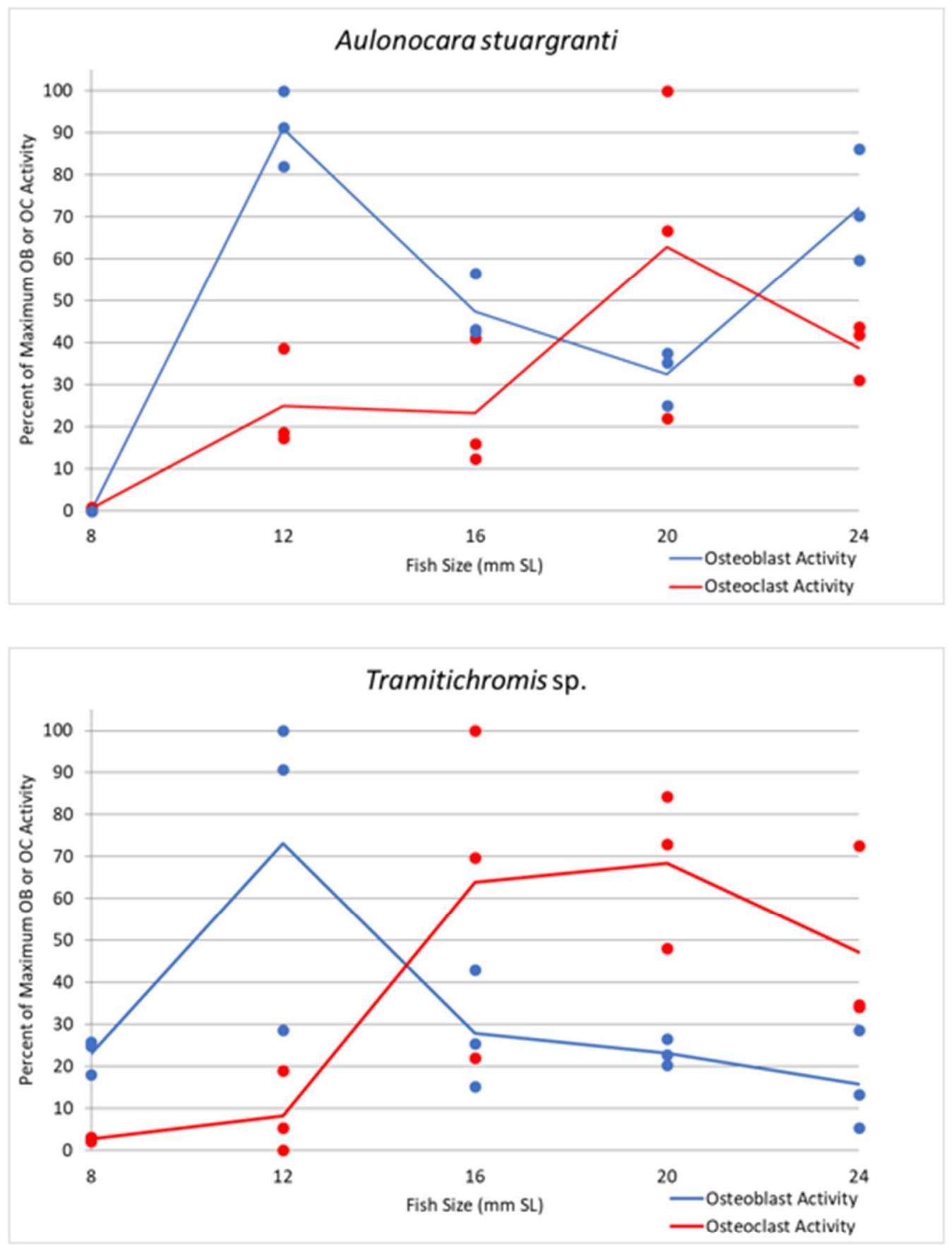

Figure 27. Osteoblast and osteoclast activity through ontogeny in Aulonocara stuartgranti and Tramitichromis $\mathrm{sp}$. The osteoblast (bone deposition) and osteoclast (bone resorption) activity in Aulonocara and Tramitichromis. Each data point represents the percentage of the maximum total osteoblast or osteoclast activity in all four quadrants at all 29 positions for each individual fish ( $n=3)$. The solid line represents the trend in the mean for all three fish at each fish size $(8,12,16,20,24 \mathrm{~mm} \mathrm{SL})$. See Figures 14, 17, and text for additional explanation. 

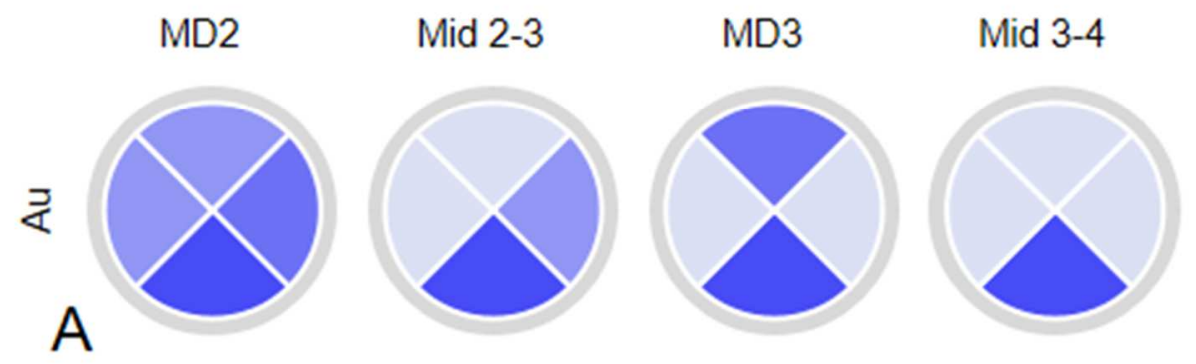

MD4
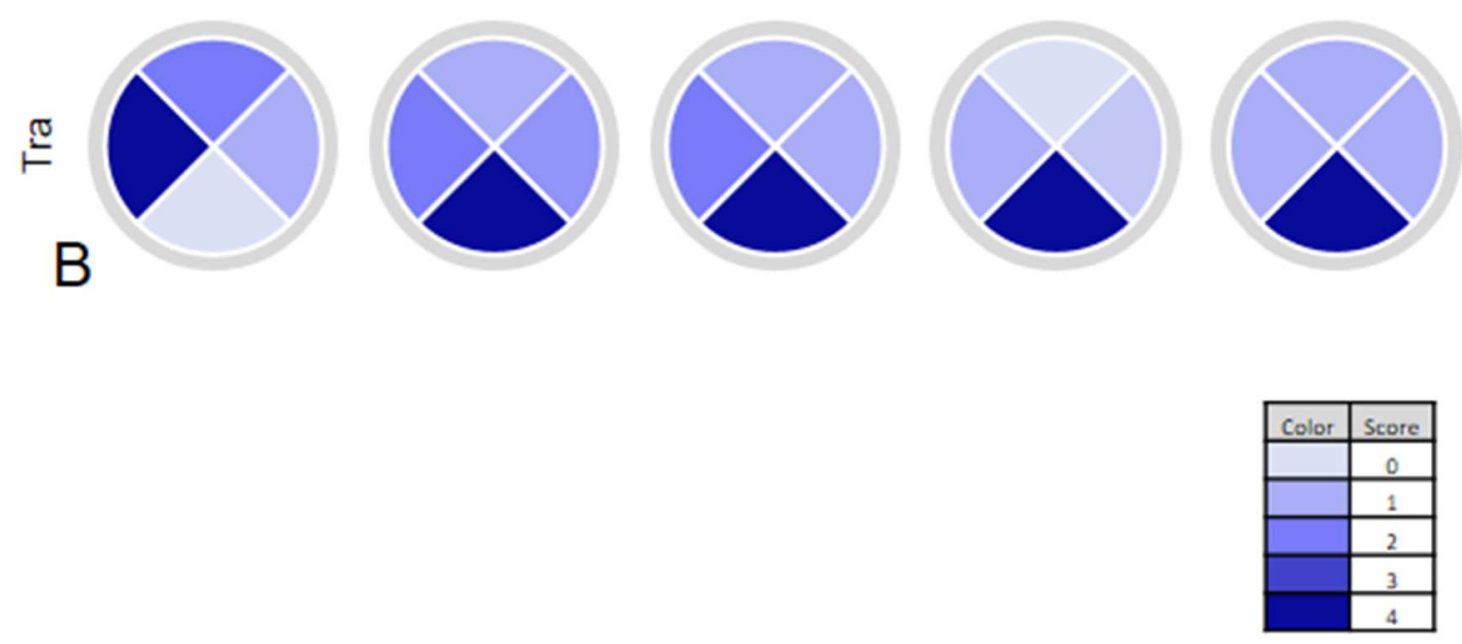

Figure 28. Neuromast-centered canal morphogenesis in $12 \mathrm{~mm} \mathrm{SL}$ Aulonocara stuartgranti and Tramitichromis sp. In $12 \mathrm{~mm}$ SL Aulonocara stuartgranti (above), osteoblasts are active in the canal floor in neuromast associated regions but not in inter-neuromast regions. However, in $12 \mathrm{~mm}$ SL Tramitichromis sp. (below), osteoblast activities are consistent along the length of the canal. 

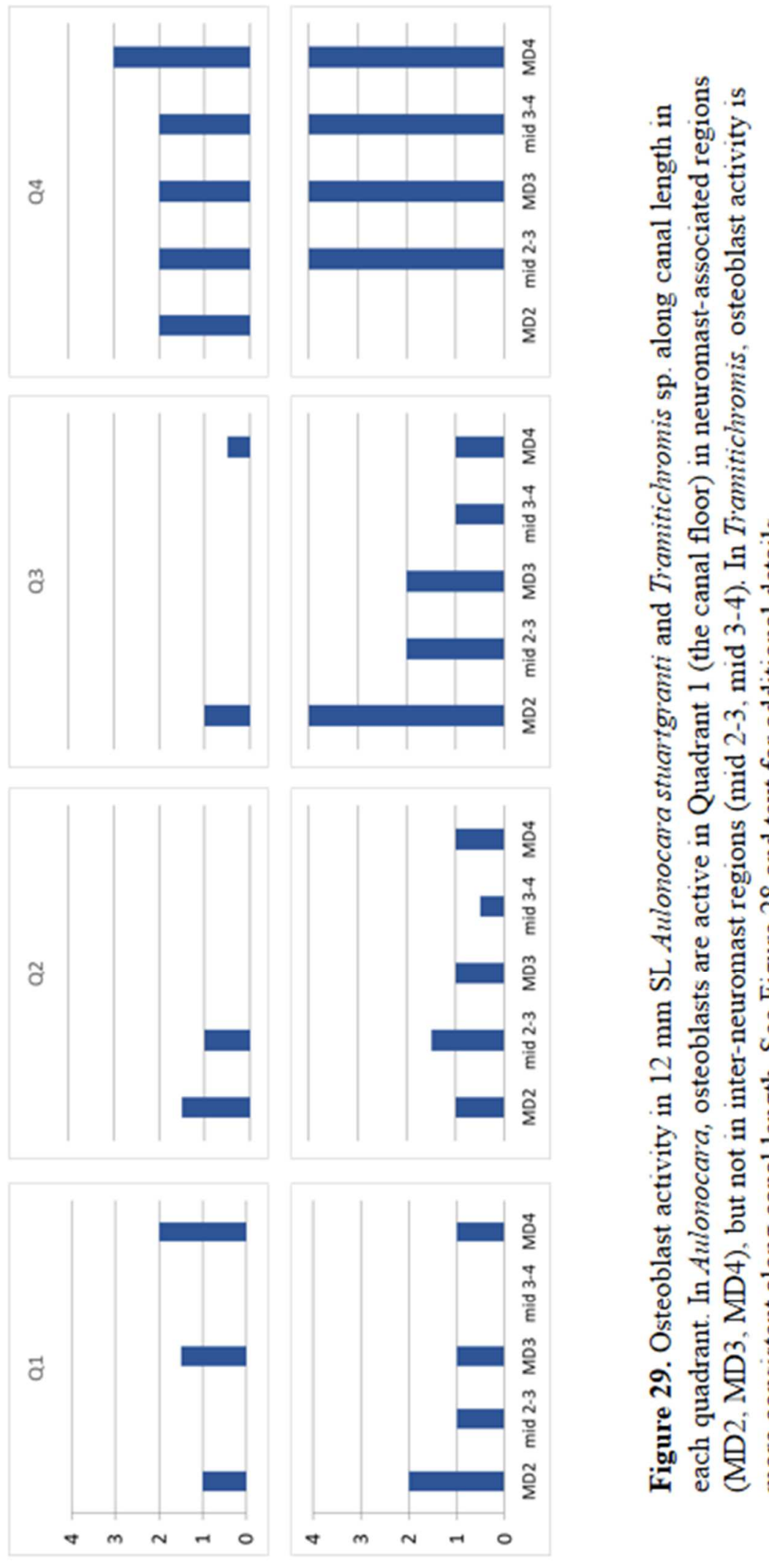

के

岕

ธิ $\Xi$

흥 ฮิ

은

흘

ثิ

길

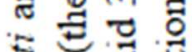

들.

站节的

홀 운

密芯范

ชิ $\cong \tilde{\exists}$

잉

氖造

స્さ

幽光苛

范芯芯

기웛

ヨ。苛

ㄴ. 0 프

어ㅇㅝㅣ

ฮิ

记

홍휴

范芯范

월롱

总芯是

DגDsouoln 